\title{
OPTICAL METAMATERIALS BY BLOCK COPOLYMER SELF-ASSEMBLY
}

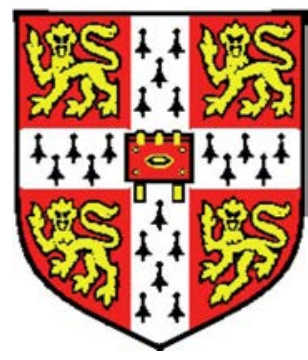

Stefano Salvatore

Department of Physics

University of Cambridge

A thesis submitted for the degree of

Philosophiae Doctor

November 2013 


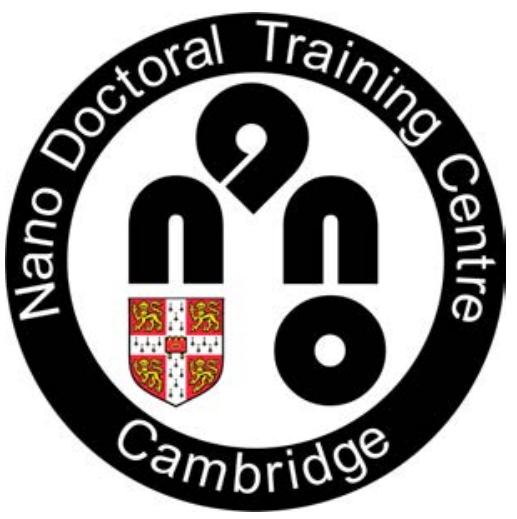

This work was supported by the EPSRC Cambridge NanoDTC, EP/G037221/1 
This dissertation is the result of my own work and includes nothing which is the outcome of work done in collaboration except where specifically indicated in the text. I declare that no part of this work has been submitted for a degree or other qualification at this or any other university. This dissertation does not exceed the word limit of 60,000 words set by the Physics and Chemistry Degree Committee.

Stefano Salvatore

November 2013 


\section{Acknowledgements}

I am not going to write a long list to acknowledge everyone who has been part of my life and my work during the PhD. I have met extraordinary people and friends but i feel that would diminish the weight i would like to give to the thank you to Ulli, Jeremy and Silvia. Neither i feel obliged to acknowledge my supervisors, but Ulli really deserves all my gratitude. He has been incredibly supportive during my $\mathrm{PhD}$ and its rushed conclusion. I feel lucky i have been supervised by a professor that considers paramount the interest of his students. It has been a pleasure to work with him and engage in numerous scientific discussions. His way of approaching work and life at work is just unique.

Part of the reasons why i chose this specific $\mathrm{PhD}$ project was for the opportunity to collaborate with prof. Jeremy. He is an extraordinary motivating and enthusiastic person. Able to inspire me in any conversation and a true drive in my work. I should really thank him from being a guide through the whole NanoDTC programme.

A special thank you shall go to Silvia, who has been for me in these years a colleague, an advisor, a guide and, mostly, a true friend. We engaged in uncountable vibrant scientific discussions, often with opposite opinions. While writing my thesis I have appreciated how valuable they were. My PhD would have been harder without her contribution and a more ordinary experience without her friendship. 


\begin{abstract}
This thesis explores the fabrication processes and the optical characterization of a metamaterials made by block copolymer self-assembly. Optical metamaterials are artificial systems designed to produce optical properties that may not be found in nature. They gain their properties not from their chemical composition but rather from their desig structure that affects the interaction with electromagnetic waves, producing an optical response different from the constituent material. The structural features have sub-wavelength size so that the metamaterial is perceived homogeneous by the incident waves and the electromagnetic response is expressed in terms of homogenized material parameters.

In this work such structural features will be fabricated well below optical wavelengths by metal replica of a gyroid morphology generated by block copolymer self-assembly.

Block copolymers consist of two or more chemically different polymers that are covalently tethered. Self-assembly in such systems is driven by enthalpy reduction through microphase separation that minimizes unfavourable interfaces, leading to a range of potential morphologies. The gyroid morphology consists of a continuous three dimensional structure with constant mean curvature and chiral directions.

The gold gyroid effectively behaves as a metamaterial with its own distinct optical characteristics: a reduced plasma frequency and highly enhanced transmission, an hall mark of optical metamaterials. The optical characterization discussed in this dissertation showed good agreement with finite difference time domain (FDTD) calculations and analytical models.
\end{abstract}


The optical properties of the fabricated metamaterials were successfully tuned by modifying the structural dimensions and the surrounding mediums. The transmission efficiency was, then, further enhanced by fabricating an hollow gyroid structure with increased surface area. The hollow gyroid enabled also the fabrication of composite metamaterial employing the combination of different metals in the 3D continuous structure.

Next, flexible and stretchable metamaterial were fabricated by infiltrating an elastomer around the gyroid network, paving the way for practical applications.

Finally, the gyroid metamaterial was used as vapour sensor, exploiting the regular pore size and the variation of the optical response with surrounding media. 


\section{Contents}

Contents vi

1 Introduction 1

2 Background 4

2.1 Block copolymers . . . . . . . . . . . . . . . 4

2.1.1 Triblock copolymer morphologies . . . . . . . . . . . . 6

2.1.2 Gyroid morphology . . . . . . . . . . . . . . . . . 7

2.1.3 Block copolymer applications . . . . . . . . . . . 10

2.2 Metamaterials . . . . . . . . . . . . . . . . . 13

2.2.1 Chiral metamaterial . . . . . . . . . . . . . . 15

2.2 .2 Surface plasmons . . . . . . . . . . . . . . . . . . 16

2.2.3 Gyroid theoretical models . . . . . . . . . . 17

3 Gyroid metamaterial fabrication $\quad 20$

3.1 Introduction . . . . . . . . . . . . . . . . . 20

3.2 Substrate preparation . . . . . . . . . . . . . . . . 21

3.3 I-S-O triblock copolymer . . . . . . . . . . . . . . . . . . . . . . 22

3.3 .1 Thermal annealing . . . . . . . . . . . . . . 23

3.3.2 Drying-annealing . . . . . . . . . . . . . . . 24

3.3.3 Solvent vapour annealing . . . . . . . . . . . . 26

3.4 PFS-b-PLA diblock copolymer . . . . . . . . . . . . . . . 27 
3.5 Metal deposition . . . . . . . . . . . . . . . . . 28

3.6 Film morphology control . . . . . . . . . . . . . . . . . . . 29

3.7 Conclusions . . . . . . . . . . . . . . . . . . 30

4 Gyroid metamaterial characterization $\quad 34$

4.1 Introduction . . . . . . . . . . . . . . . . . 34

4.2 Transmission electron microscopy . . . . . . . . . . . . 35

4.3 Electric characterization . . . . . . . . . . . . . . . 36

4.4 Optical properties . . . . . . . . . . . . . . . . 37

4.4.1 Single gyroid . . . . . . . . . . . . . . . . 37

4.4 .2 Film thickness effects . . . . . . . . . . . . . . 43

4.4 .3 Disorder effects . . . . . . . . . . . . . . . . 45

4.4.4 Double and Silver gyroids . . . . . . . . . . . . 46

4.5 Conclusions . . . . . . . . . . . . . . . . . . . . . . 49

5 Tuning methods $\quad 51$

5.1 Introduction . . . . . . . . . . . . . . . . . 51

5.2 Unit cell control . . . . . . . . . . . . . . . . . . . . 53

5.3 Filling fraction variation . . . . . . . . . . . . . . 54

5.4 Surrounding medium effects . . . . . . . . . . . . 57

5.5 Conclusions . . . . . . . . . . . . . . . . . . . 59

6 Hollow gyroid $\quad 60$

6.1 Introduction . . . . . . . . . . . . . . . . . 60

6.2 Hollow gyroid fabrication . . . . . . . . . . . . . . 61

6.2.1 Optical properties . . . . . . . . . . . . . 61

6.3 Carbon hollow gyroid . . . . . . . . . . . . . . . . . . 67

6.4 Inverse gyroid . . . . . . . . . . . . . . . . . . . 68

6.5 Conclusions . . . . . . . . . . . . . . . . . 70

$\begin{array}{lll}7 & \text { Flexible and stretchable gyroid metamaterials } & 71\end{array}$

7.1 Introduction . . . . . . . . . . . . . . . . . 71

7.2 Stretchable metamaterial . . . . . . . . . . . . . . 71

7.3 Flexible metamaterial . . . . . . . . . . . . . . . . 74 
7.4 Conclusions . . . . . . . . . . . . . . . . . . 78

$\begin{array}{llr}8 & \text { Metamaterial sensors } & 79\end{array}$

8.1 Introduction . . . . . . . . . . . . . . . . . . . . 79

8.2 Capillary condensation . . . . . . . . . . . . . . 80

8.3 Solvent vapour sensing . . . . . . . . . . . . . . . 81

8.4 Conclusions .......................... 84

$\begin{array}{ll}\text { Appendix } & 85\end{array}$ 


\section{Chapter 1}

\section{Introduction}

"Either a body absorbs light or it reflects or refracts it or does all these things. If it neither reflects nor refracts nor absorbs light, it can not itself be visible"

(The Invisible Man, George H. Wells, 1897).

In G. Wells' famous novel, a scientist invented a way to change the refractive index of his body to that of air so that it adsorbed and reflected no light, becoming invisible. Besides its science fiction, it is interesting to consider how the optical properties of matter can be controlled and engineered. The optical properties of any material result from the electric and magnetic responses of its constituent atoms to the electromagnetic waves of light. Such response can be tailored only to some extend by adjusting the chemical composition.

However, recently it has been realized that the internal microstructure of a material can be just as important as the chemistry in determining its optical properties. In fact, by exploiting both chemistry and microstructure, materials can be produced with properties never found in nature, and can, in principle, alter light propagation leading to a cloaking device. This new class of materials, called metamaterials, achieve this effect through a structure that is much smaller than the wavelength of radiation in the region of interest, so that the electromagnetic radiation interacts with the metamaterial as if it was a continuous material.

In this way, the overall properties are driven by the electric and magnetic field induced by the engineered structure, producing a response that may be different from the constituent material. 
Several structures have been proposed in the last few years and a wide range of outstanding properties have been successfully produced in the microwave region. Nonetheless, a metamaterial active at optical wavelengths (from about 400 to $700 \mathrm{~nm}$ ) is radically more difficult to produce, due to the required scale of the structural features.

In this work a gold metamaterial is fabricated with feature sizes below $10 \mathrm{~nm}$ and, remarkably, it is created by self-assembly. The fascinating architecture of this metamaterial, called gyroid, is obtained by block copolymer self-assembly.

The first chapter of this dissertation will focus on the theoretical background of the block copolymer self-assembly and on the metamaterial theory. I will also dedicate a large section to the gyroid architecture and to its peculiar geometrical properties.

I will begin the discussion of my experimental results in Chapter 2 describing the fabrication process. This Chapter highlights the largest amount of work that went into the fabrication of the gyroid metamaterials and to the development of a solid and reproducible fabrication process.

The optical characterization will then be discussed in Chapter 3. The gyroid metamaterial has raised in the past few years increasing attention in the metamaterial research community. Although there are no other research groups experimentally producing gyroid metamaterials, there have been several theoretical studies predicting interesting properties of this structure. These theoretical results will often be mentioned through out this dissertation to support my experimental results and help the understanding of the gyroid optical properties.

As already mentioned, the properties of optical metamaterials are strictly connected to their structure. In Chapter $4 \mathrm{I}$ will show the tuning mechanisms to tailor the optical properties of the gyroid metamaterial by altering the structural parameters and the surrounding medium.

A further development in the gyroid fabrication has led to the so-called hollow gyroid, presented in Chapter 5. The gyroid morphology was produced with an additional internal interface, giving rise to an improvement of the optical properties and to an extra degree of freedom in designing an optical metamaterial.

In Chapter 6 I will show the fabrication and the optical properties of a flex- 
ible and stretchable metamaterial produced by infiltrating an elastomer into the gold gyroid network. Although the optical properties varied only marginally, the importance to produce a metamaterial not confined to flat films is significantly relevant for any practical use.

An application of the gyroid metamaterial will then presented in Chapter 7 . The gold gyroid was used as vapour sensor, exploiting the high monodispersity of the pore size and the strong optical variation with respect to different surrounding media.

Finally, the conclusions of this work will be summarized and an outlook will be given in the final chapter with the hope to get closer to the vision of George Wells and his novel. 


\section{Chapter 2}

\section{Background}

This work is based on the combination of two distinct subjects: the self-assembly of block copolymers in thin films and optical metamaterials.

In this Chapter I will give a brief introduction of these two fields and of their recent research progress.

\section{$2.1 \quad$ Block copolymers}

Polymers are a class of macromolecules composed of many repeating subunits, defined as monomers, linked by covalent bonds. Polymers can be classified into two types: homopolymers composed of only one repeating monomer unit, and copolymers derived from two or more types of monomer units. In turn, copolymers can be classified based on how these units are arranged along the chain. The arrangement can generate: alternating copolymers with regular alternating $\mathrm{A}$ and B units (e.g. A-B-A-B-...), periodic copolymers with A and B units arranged in a repeating sequence (e.g. (A-B-B-A-B)n), statistical copolymers in which the sequence of monomer residues follows a statistical rule, and block copolymers ?. In the block copolymer subclass, two or more homopolymers are covalently arranged into distinct blocks ?. The simplest example is an A-B block copolymer with two covalently tethered homopolymers. Block copolymers with two or three distinct blocks are called diblock copolymers and triblock polymers, respectively (Figure 2.1). 


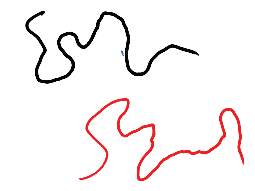

Homopolymers

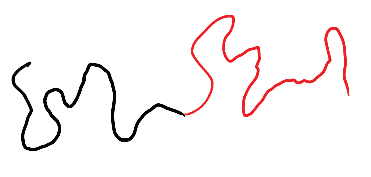

Diblock copolymer

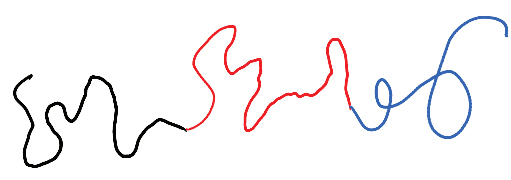

Triblock copolymer

Figure 2.1: Homopolymers and block copolymers architectures. Polymers are a class of macromolecules that are composed of many repeating subunits. Homopolymers consist of a single type of monomer, while linear block copolymers are chains containing sequences of different homopolymers.

Block copolymer can be synthesized by living polymerization ?, a technique that enables the sequential addition of monomers and the synthesis of functionalended polymers by selective termination allowing the additive synthesis of additional blocks.

An important property of block copolymers is their ability to self-assemble into periodic nanostructures. To understand the block copolymer self-assembly let us first consider a mixture of two separate immiscible homopolymers A and B. The minimization of unfavourable interfaces drives the homopolymers to blend to macrophase separation into coexisting bulk phases, similarly to water and oil mixtures. When two immiscible homopolymers are covalently bonded to form an A-B block copolymer, the length scale of the phase separation is limited by the polymer chain length, leading to microphase separation.

In the block copolymer melt, the enthalpic forces, driving the interface minimization, are counterbalanced be entropic forces that encourage the reduction of chain stretching ?. The affinity between two polymers is defined by the FloryHuggins interaction parameter $\chi$. In addition to the Flory-Huggins parameter, the phase behaviour of block copolymers is controlled by the degree of polymerization $N$ and the volume fraction of each block ( $f_{\mathrm{a}}$ and $f_{\mathrm{b}}$ in a diblock copolymer).

For volume fractions $f_{\mathrm{a}} \simeq f_{\mathrm{b}}$, the interface minimization drives the phase separation to lamellae containing alternating the A and B blocks. For volume fractions $f_{\mathrm{a}} \gg f_{\mathrm{b}}$ the best geometrical solution to minimize the polymer interface 
leads to the formation of spheres containing the polymer with the lower volume fraction, embedded in a major phase of the other polymer.

The equilibrium phases can therefore be mapped as a function of the volume fraction $f_{a}$ and the $\chi N$ parameter in a phase diagram, shown in Figure 2.2.
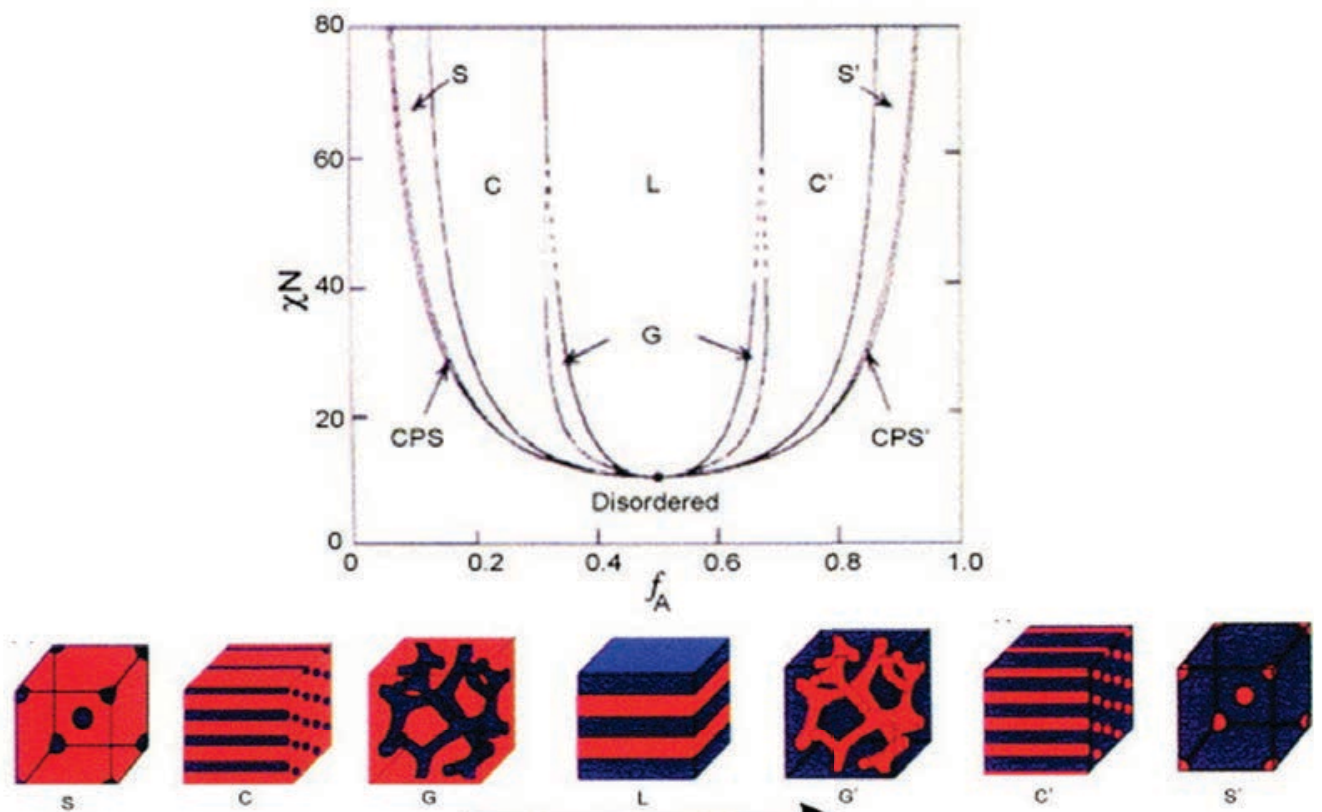

Figure 2.2: Phase diagram for linear $\mathrm{AB}$ diblock copolymers with the regions of stability for cylinders (C), spheres (S), lamellae (L), gyroid (G), close packed spheres (CPS), and disordered phase. Adapted from?.

\subsubsection{Triblock copolymer morphologies}

The addition of a third block to a linear block copolymer gives rise to several new potential architectures, some of which are shown in Figure 2.3, with others still under investigation ???.

In the gyroid morphology, the blocks forming the two interwoven networks are composed of distinct polymers, denoted as minority phases, each forming a so-called single gyroid. The largest part of the work presented in this dissertation focuses on gyroid formed by triblock copolymers, where one of the minority phases 

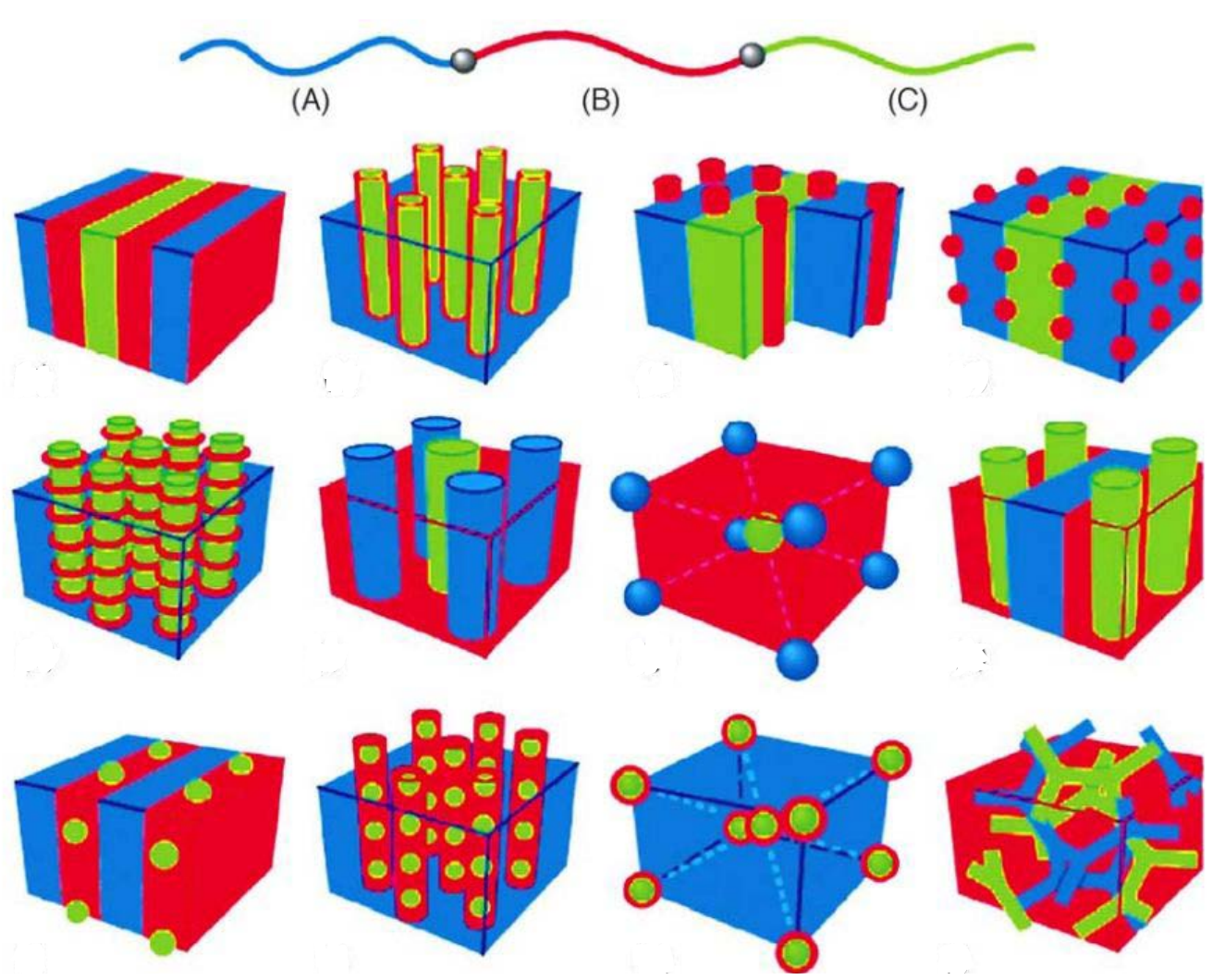

Figure 2.3: Morphologies for linear ABC triblock copolymers. The phases are coloured as shown by the copolymer blocks at the top, with block types A, B and $\mathrm{C}$ confined to regions colored blue, red and green, respectively. Adapted from ?.

was selectively etched and backfilled by electrodeposition, producing a metal single gyroid.

The single gyroid has strong chirality in certain directions, with helices visible in the [111] and [100] orientations as shown in Figure 2.4. In these directions we can distinguish two helices with opposite chirality and different radii.

\subsubsection{Gyroid morphology}

The gyroid phase observed in diblock copolymers is a structure consisting of two interwoven continuous networks, containing the polymer with lower volume fraction, embedded in a matrix formed by the majority block. Since both minority and majority phases are continuous in all three dimensions the structure is termed 
a

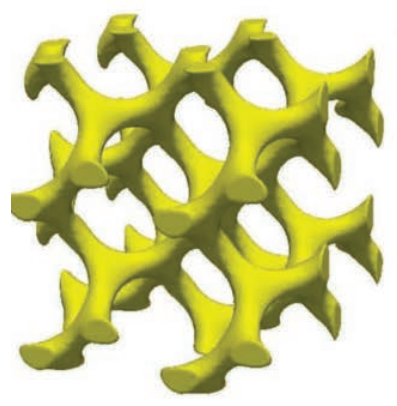

b

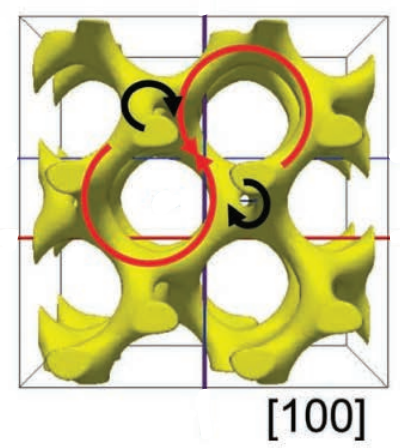

C

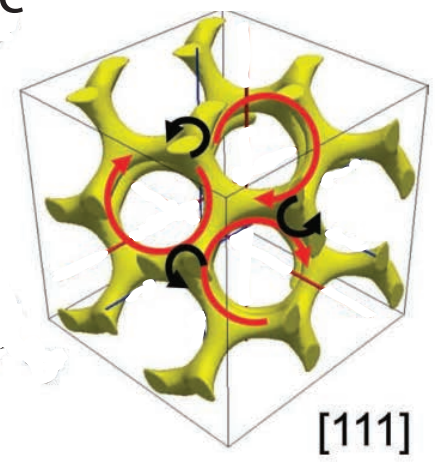

Figure 2.4: (a) Perspective view of the gyroid structure with $10 \%$ filling fraction. (b) View of the chiral [100] direction with the two opposite helices indicated by the black and red arrows. (c) View of the chiral [111] direction. Adapted from ?.

bicontinuous.

The term "gyroid" was coined in 1970 by Alan Schoen ?, a mathematician who discovered the family of triply periodic minimal surfaces (i.ewith zero mean curvature everywhere), which are now named after him. The "Schoen G" or "gyroid surface" is a triply periodic minimal surface that divides the space into two separate twisting volumes as shown in Figure 2.5a.
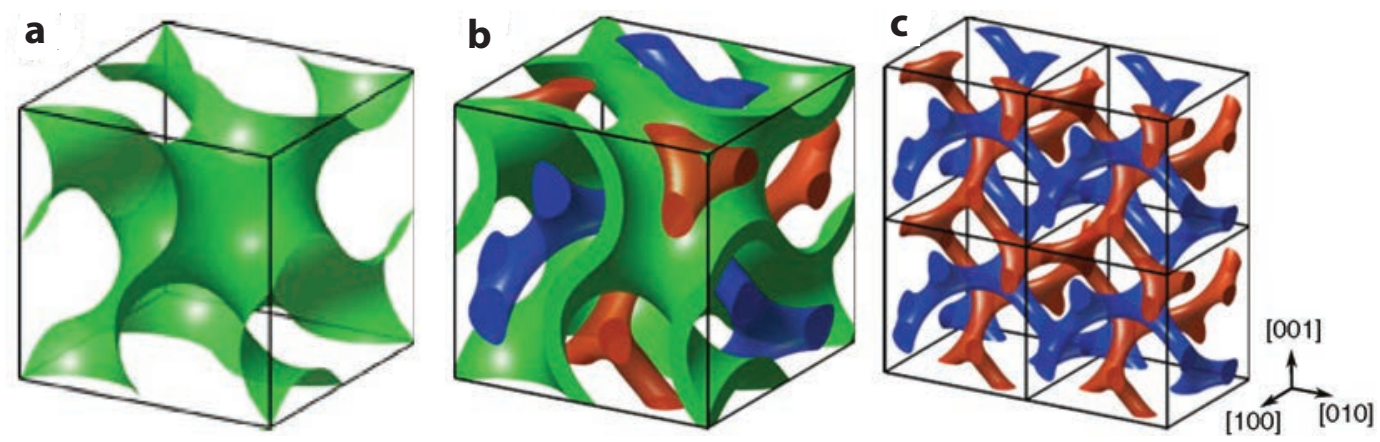

Figure 2.5: (a) "Schoen G" or "gyroid surface".(b) A schematic of the the double gyroid unit cell showing the majority phase equivalent of the gyroid surface (green) and the two gyroid networks (blue and red). (c) Four cubic unit cells of the double gyroid network with a volume fraction of $12 \%$. Adapted from ?.

The majority phase of the gyroid block copolymer is equivalent to the gyroid 
surface with a given finite thickness, by simultaneously expanding of the two surfaces on both sides. Space is consequently divided into three non-intersecting volumes. The "central volume" forms the majority phase, embedding two distinct non-intersecting gyroid networks. The two gyroid network morphology will be hereafter called double gyroid.

Why polymers select this non trivial morphology is an interesting issue that can be addressed by examining the concept of minimal surface. A minimal surface is a surface that minimizes the total surface area under some constraint. For example, under the constraint of connecting two parallel rings, the geometry that minimizes the surface area corresponds to the catenoid minimal surface. Similarly, the Schwartz P triply periodic minimal surface connects a cubic array of rings, as shown in Figure 2.6. The gyroid surface can be seen as a tessellation of gyrating catenoids associated with the P-surface ?.

In the gyroid block copolymer system, the unfavourable interaction between polymers leads to the segregation of majority block in the the central matrix volume that, as a minimal surface, represents an optimized geometry which minimizes interface interactions.

a

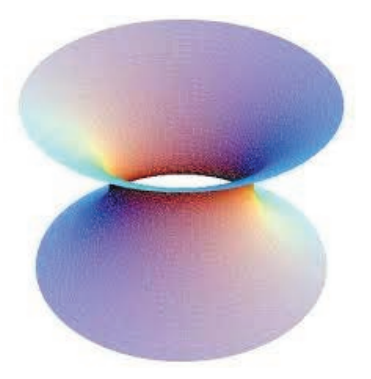

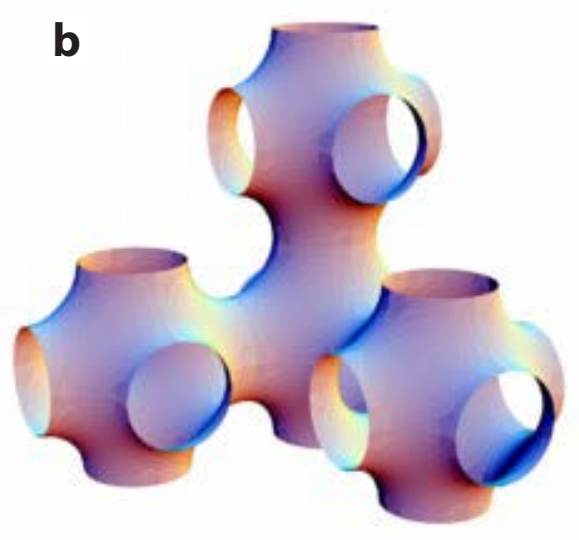

Figure 2.6: (a) Catenoid minimal surface derived by minimizing the connecting surface of two parallel rings. (b) Schwartz $\mathrm{P}$ periodic minimal surface produced by connecting a cubic array of rings.

Interestingly, the gyroid geometry is also found in nature in several systems 
in addition to block copolymers. In 1983 it was found in some surfactants, where lipid appeared to form a single bilayer continuous in three dimensions, separating two networks of water with a Schwartz G geometry ?.

Gyroids have been observed in biological structural coloration in butterfly wing scales ??. In these systems the gyroid structure has lattice size one order of magnitude higher than in the block copolymers, giving rise to photonic crystals. Gyroid structures are also occasionally found inside cells ?.

The gyroid surface can be trigonometrically approximated by the equation:

$$
\sin \left(\frac{2 \pi}{L} x\right) \cos \left(\frac{2 \pi}{L} y\right)+\sin \left(\frac{2 \pi}{L} y\right) \cos \left(\frac{2 p i}{L} z\right)+\sin \left(\frac{2 \pi}{L} z\right) \cos \left(\frac{2 \pi}{L} x\right)=t,
$$

where $L$ is the cubic unit cell and $t$ is equal to 0 .

For increasing $t$ with $0<|t| \leq 1.413$ the gyroid surface is monotonically reduced with an increase of volume of one of the two space regions divided by the gyroid surface, on the expense of the other. For $1.413 \leq t$ the gyroid surface is no longer connected and will be neglected in the following discussion.

Let us consider one of the two gyroid networks formed by the minority phase. Its surface can be described by Eq. 2.1. For $t=0$, the single gyroid network occupies exactly one half of the total volume, i.eit has filling fraction $f=50 \%$. For $t>0(t<0)$ the filling fraction decreases (increases) monotonically and the surface of the gyroid network has no longer zero but an increasing (decreasing) constant mean curvature.

The surface area of the gyroid is related to its filling fraction. For the double gyroids the surface area per unit volume is plotted in Figure 2.7. In the single gyroid the relation of surface area and filling fraction is analogous, with a maximum for $f=50 \%$ and a symmetric decaying for $f>50 \%$

\subsubsection{Block copolymer applications}

Block copolymers have received much attention in the past two decades with increasing applications in nanotechnology. Nonetheless, their commercial interest has started over 50 years ago. Block copolymers can be found in products as 


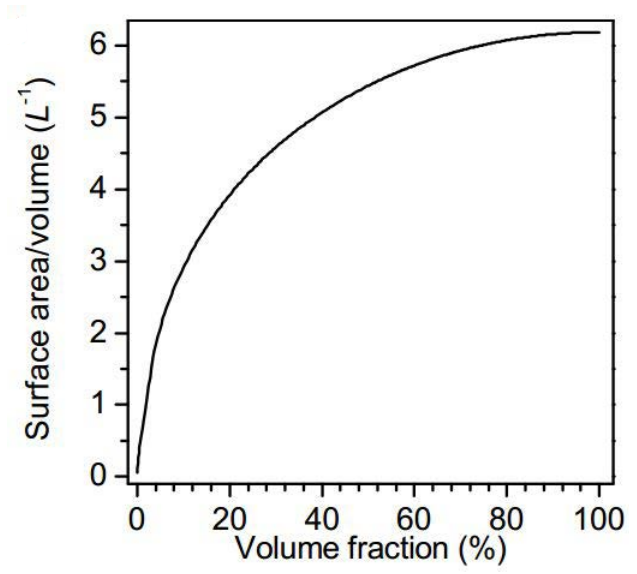

Figure 2.7: Specific surface area to bulk volume ratio in units of the cubic unit cell dimension $L$ of the double gyroid plotted versus the filling fraction. Adapted from ?.

shoe soles, tire treads, adhesive tape and asphalt additives that combine hightemperature resilience and low-temperature flexibility. The importance of block copolymers can be seen in their wide array of combined properties made possible by different conjuncted polymers.

The applications in nanotechnology are related to the scale of the microphase separation and to the tunability of the microphases, regulated simply by the molecular weight and composition, and to the wide range of chemical and physical properties of the constituent polymer blocks.

Block copolymer films have been used principally in nanolithographic etching processes, where the polymer phases function as a mask with high density of features ?. They have also been utilized to develop periodic photonic band gap materials. The first example was a simple 1D photonic crystal made with high molecular weight lamellae ?.

Other block copolymer applications in nanotechnology include porous membranes based on the large surface/volume ratio, low density and pore size monodispersity ?.

The replication of one or more phases to use a block copolymer as sacrificial template has been widely demonstrated. Metals are typically deposited by elec- 
troless deposition, while oxides and other dielectrics are produced by atomic layer deposition (ALD) or sol-gel chemistry ?.

The replication of the gyroid morphology into metals was also achieved by electroless deposition of nickel for catalytic applications ? and by sol-gel chemistry to create a highly porous $\mathrm{SiO}_{2}$ structure with a reduced refractive index.

Gyroid block copolymers have found applications in supercapacitors ?, solar cells ? and nanoporous membranes ?, and represent to date a versatile structure for an increasing number of applications.

However, the main limitation of the block copolymers applications in nanotechnology lies with the fact that, while the self assembled structure is locally very precise, it is difficult to control its order on larger scales. The domain size of the periodic ordering are typially of several tens of the lattice constants, limiting the long-range order to $2-3 \mu \mathrm{m}$.

In the next Chapter I will discuss a fabrication process that enables the ordering of the gyroid block copolymer into domains of several hundreds of microns, an essential requirement for engineering applications and optical characterizations. 


\subsection{Metamaterials}

Metamaterials are artificially structured materials which are engineered to gain their properties not only from their composition, but from their design. Their geometry, size and arrangement can affect the waves of light or sound in a unconventional manner, giving rise to properties which are not available in any other bulk material (the word 'meta' means 'beyond' in Greek, and in this sense the name metamaterial refers to 'beyond conventional materials'). The structural units have dimensions much smaller than the wavelengths of interest, so that the metamaterial is perceived homogeneous by the incident waves and their electromagnetic response is expressed in terms of homogenized material parameters.

Sought-after properties in metamaterials are the simultaneous negative electric permittivity $\epsilon$ and magnetic permeability $\mu$. This leads to a negative refraction as demonstrated by Veselago in 1968 ?. Negative index metamaterials have the potential to form superlenses, overcoming the diffraction limit, or to enable novel optical effects, including cloaking ??

Although negative permittivity is quite common in metals at optical wavelengths, it is very unusual to find natural materials that exhibit a magnetic response at terahertz or higher frequencies?.

However, a metamaterial made of a conductive but not-magnetic material designed as a split-ring resonator (SRR) can display a magnetic response and negative permeability at the resonance frequencies of this oscillator, as first predicted by John Pendry in 1999 ?. A single cell SRR consist of a pair of enclosed loops with splits in opposite sides as shown in Figure 2.8. They represent a paradigm example of a metamaterial system. A magnetic wave penetrating the metal rings produces an oscillating current flow that induces local magnetic dipole moments giving an effective magnetic response and thereby an engineered permeability.

When a SRR array is combined with an array of conducting wires it can create a medium with simultaneous negative permeability a permittivity.

Smith and coworkers showed evidence of negative index refraction with ring resonators in 2001 ?. The first metamaterials were demonstrated at microwave frequencies and then scaled to the mid-infrared ?. However, further down-scaling requires a different approach as a consequence of the strong deviation of metals 


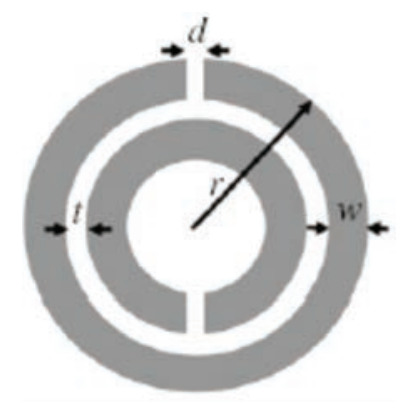

Figure 2.8: Split-ring structure proposed by Pendry et al. ?

from ideal conductors at high frequencies?. Moreover, to extend metamaterials to optical frequencies requires scaling the constituting features from the millimetre size down to the nanometre scale.

Several metamaterial architectures have been proposed in the past decade, some of which are summarized in Figure 2.9. The operating frequency of optical metamaterials have already reached visible wavelengths, and negative refraction has been proved for light wavelengths between 720 and $760 \mathrm{~nm}$.

However, some critical problems still remain unsolved. The main drawback for most potential applications of metamaterials are the high losses originated from the metal in the structure. These are a critical problem mainly at the infrared and optical frequencies where the metals differs from a perfect conductor. This results in high absorption and strong reduction of light transmission through the metamaterials.

Another problem in the fabrication of metamaterials is the development of truly 3D structures. The current metamaterials are generally limited to light coming from a narrow range of directions.

The proposed block copolymer based metamaterial is truly three dimensional and, as we will see in the following chapters, it displays strongly enhanced transmission, i.eit has low losees. Moreover, it is produced by self-assembly in contrast to the largely used top-down techniques of current metamaterials. 
a

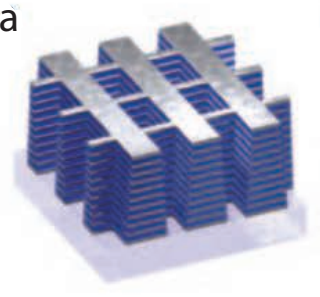

e

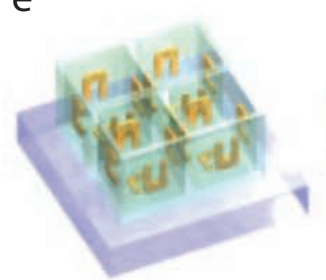

b

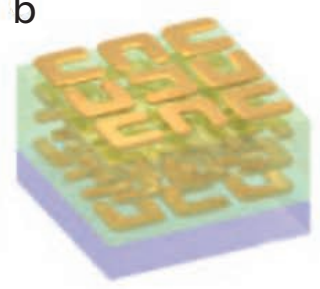

$f$

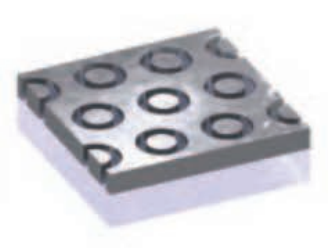

c
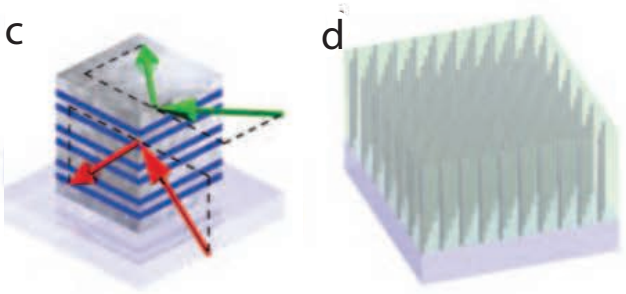

g
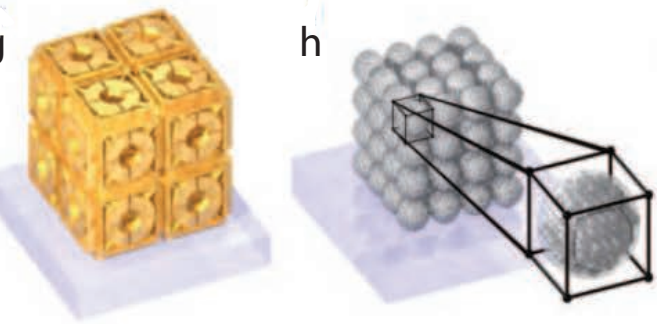

Figure 2.9: Principal metamaterial designs proposed in the past decade. (a) Fishnet structure ?; (b) Stereo metamaterial ?; (c) Hyperbolic metamaterial ?; (d) Metal-dielectric layered metamaterial ?; (e) Three dimensionally oriented SRRs ?; (f) Coaxial metamaterial ?; (g) Connected cubic-symmetry metamaterial ?; (h) Metal cluster-of-clusters array ?. Adapted from ?

\subsubsection{Chiral metamaterial}

It was recently proposed ? that a chiral structure can offer a unique route to a negative refractive index for one circular polarization. A structure is dened as chiral if it lacks any planes of mirror symmetry.

The existence of chirality breaks the degeneracy of two oppositely circularly polarized waves. The two polarized beams experience different propagation speeds in a chiral media, i.e. the refractive index is increased for one circular polarization and reduced for the other. Therefore, if the chirality is strong enough, negative refraction may occur for one circularly polarized wave even when permittivity and permeability are both positive.

The first chiral metamaterial was proposed by Pendry in his pioneering work ? as a "Swiss Roll" structure. Other theoretical works have also demonstrated negative refraction in the Thz and microwave regimes ?. The principal experimentally obtained chiral metamaterials are summarized in Figure 2.10.

The complex structure of chiral metamaterials has been a significant challenge 


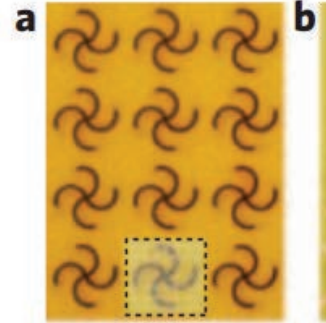

d

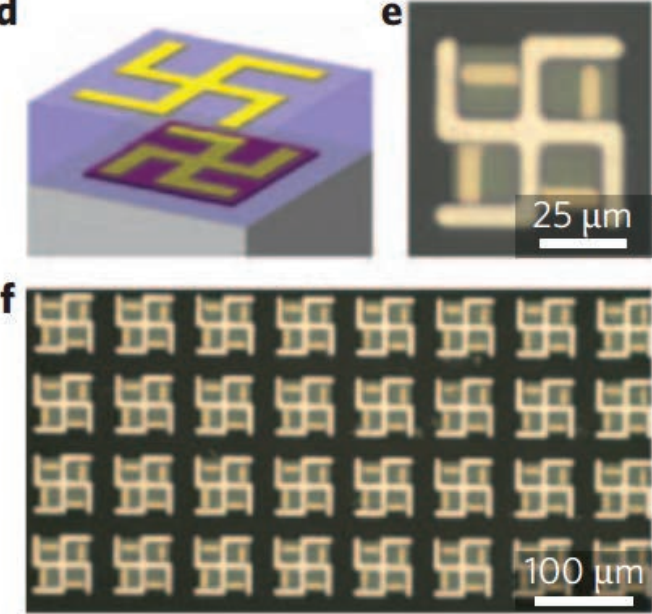

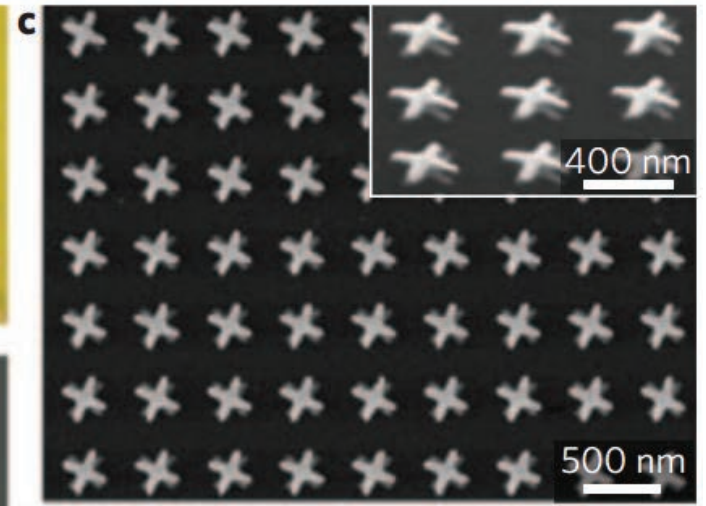

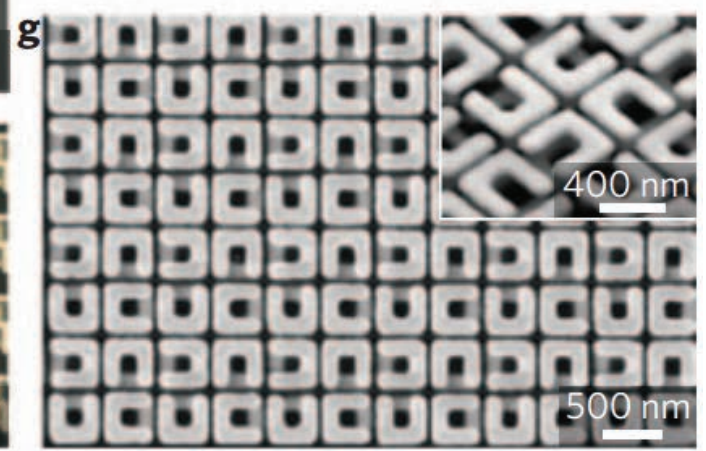

Figure 2.10: Chiral metamaterial structures proposed in the past years. (a) Rosette chiral metamaterial with negative refraction at gigahertz frequencies ?. (b) Cross-wire chiral metamaterials with negative refraction at $\mathrm{GHz}$ frequencies ?. (c) Chiral structure composed of right-handed twisted gold crosses ?. (d-f) Tunable chiral metamaterial ?. (g) Twisted U-shaped split ring resonators ?. Adapted from ?

in the miniaturization to the nanometric scale. Standard top-down approaches such as lithography techniques are not ideal to fabricate complex three dimensional chiral structures whereas the self-assembly of gyroid block copolymer is an ideal candidate to produce a chiral metamaterial.

\subsubsection{Surface plasmons}

Metamaterials with features on the nanometre scale derive their optical properties from surface plasmon waves. These are collective oscillations of free electrons on the surface of metallic interfaces. The free electrons dominate the interaction 
of light with metals. The optical electromagnetic field produces a force that displaces these weakly bound electrons. Since the electrons are negatively charged particles in a background of positive charges, the electron movement produce a restoring force. The free electrons can be considered as optically driven oscillators with with a natural frequency of oscillation known as the plasma frequency. This is given by:

$$
\omega_{\mathrm{p}}=\sqrt{\frac{n_{\mathrm{e}} e^{2}}{\epsilon_{0} m^{*}}}
$$

where $\epsilon_{0}$ is the dielectric constant of free space, $e$ is the charge of the electrons, $n_{\mathrm{e}}$ is their number density, and $m^{*}$ is their effective mass.

The interaction of the electromagnetic field and the surface free electrons produces surface plasmons that can propagate along the surface. In contrast to the propagating nature of the surface plasmons, the field perpendicular to the surface decays exponentially with distance from the surface. The field in this perpendicular direction is said to be evanescent. In the dielectric medium above the metal, typically air, the decay length of the field is on the order of one-half of the wavelength of light, whereas the decay length into the metal is determined by the skin depth, as represented in Figure 4.9.

The skin depth of noble metals in the visible spectrum is on the order of $20 \mathrm{~nm}$ ?. As the strut thickness of the gold gyroid is only $10 \mathrm{~nm}$, the entire gyroid network acts effectively as a 'surface' and all the material is permeated by surface plasmons.

As we will see in the next chapters the effect of surface plasmons in the gyroid metamaterial enables enhanced transmission through gold gyroid films of several hundred nanometers.

\subsubsection{Gyroid theoretical models}

The gyroid morphology has received increasing attention as metamaterial structure, leading to theoretical predictions of its optical properties. However, these results are still debated.

Hur et al. ? have calculated the the photonic band structures of single and 

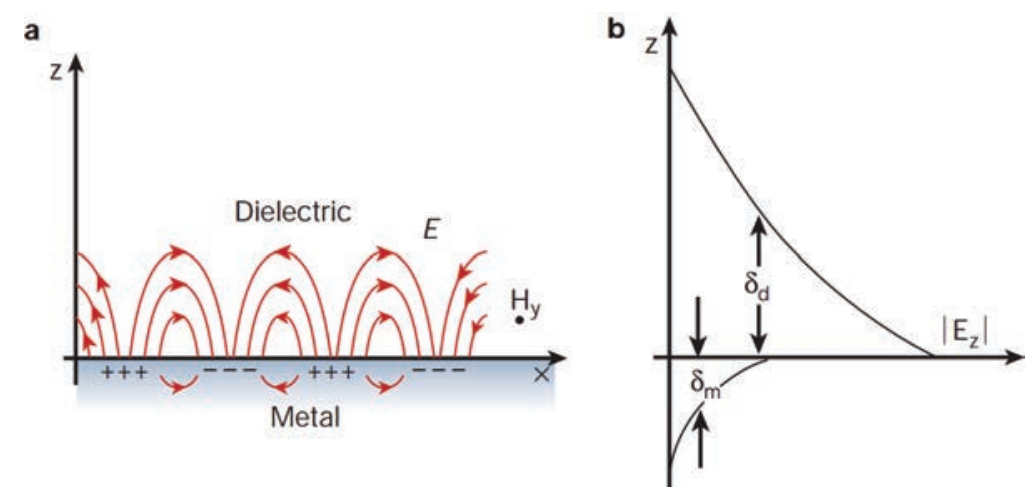

Figure 2.11: (a) Electromagnetic wave and surface charges of surface plasmons at the interface between a metal and a dielectric material. (b) The field component perpendicular to the surface is enhanced near the surface and decays exponentially with distance. Adapted from ?.

double gyroids made of gold and silver. The double gyroid morphology was predicted to form a capacitor, leading to a different light propagation mechanism than in the single gyroid morphology. The light propagation was suggested to originate from the coupled surface plasmons resonance on the closed loops formed in the double gyroid, supporting the surface plasmon propagation.

Negative refraction was predicted in silver and aluminium double gyroids, with $n$ inversely proportional to the unit cell size and the frequency range controlled by the filling fraction. According to this study, the strong absorption deriving from the inter-band transitions impede the negative refraction in gold gyroids. Moreover, in the single gyroid, the lack of a counter electrode was predicted to inhibit light propagation through the metallic gyroid network.

Oh et al. ? simulated the gyroid metamaterials by approximating the gyroid with three orthogonal helices. They identified one longitudinal and two degenerate transverse modes in the gold single gyroid. Their results suggest that the chirality may be smaller than expected as a direct consequence of the mixture of right- and left-handed helices shown in Figure 2.4.

The only experimental work on gold gyroid metamaterials was presented by Vignolini et al. in 2011 ?. It showed a strong reduction of the gold plasma frequency and circular dichroism. These results will be discussed more in detail 
in the next chapters. 


\section{Chapter 3}

\section{Gyroid metamaterial fabrication}

\subsection{Introduction}

The basic steps to produce an optical metamaterial from block copolymer selfassembly can be summarized in the following: (a) self-assembly of gyroid block copolymer in thin films; (b) selective degradation and removal of one of the minor phases; (c) back filling of the so created nanoporous template via electrodeposition; (d) removal of the remaining polymer template. Figure 3.1 shows a schematic representation of the gold gyroid fabrication in the case of single gyroid from a triblock copolymer.

The self-assembly of the gyroid triblock copolymer in thin films and the replication of one of the minor phases into gold was accomplished by Nataliya Yufa in 2009. Although this proof of principle enabled the first characterization of the gyroid gold metamaterial?, the fabrication method was lacking in reproducibility and required a better understanding of the annealing procedure and substrate preparation.

In this chapter I will describe different annealing procedures that I have established and further developments in the fabrication process. 


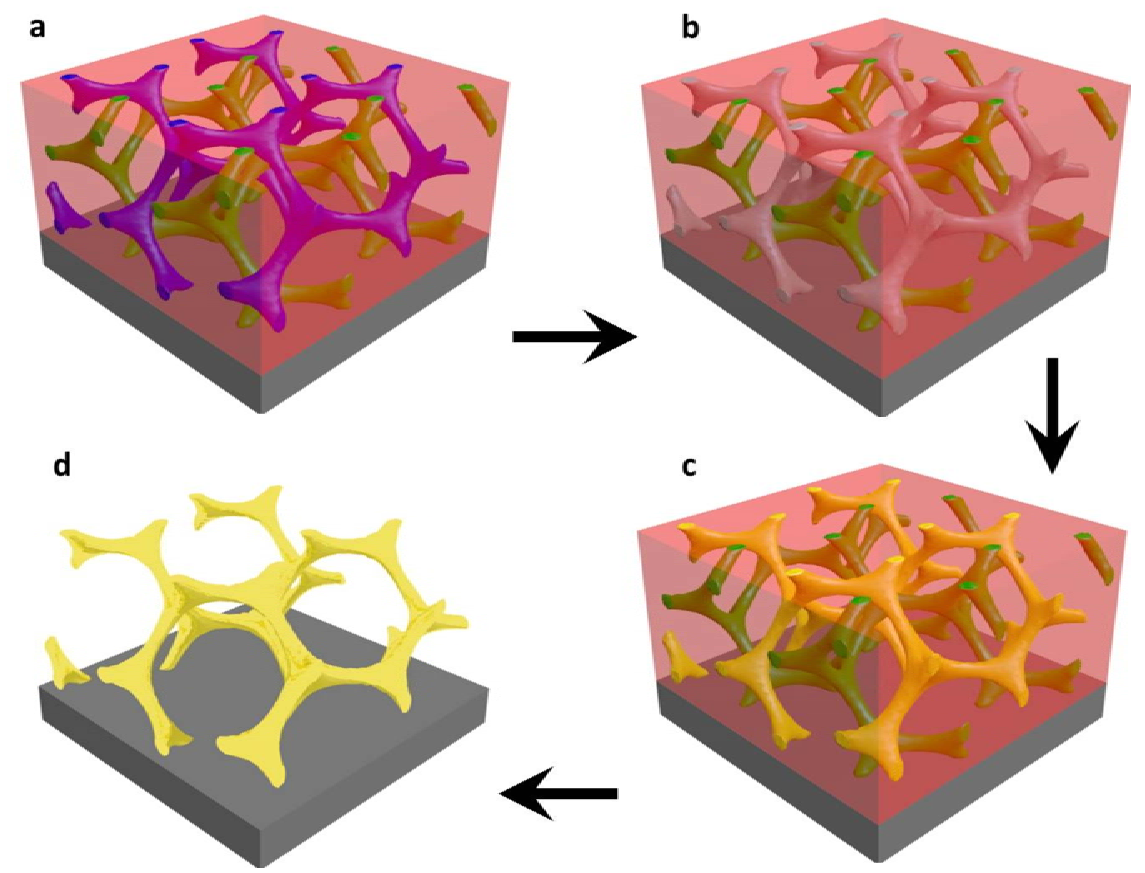

Figure 3.1: Schematic representation of gold single gyroid fabrication. (a) The three differen colours (red, blue, and green) correspond to the different blocks of the copolymer. The isoprene block (blue) is removed (b) and is then back-filled with gold (c). The final 3D continuous gold network is revealed by removal of the two remaining polymer blocks by plasma etching $(\mathrm{d})$.

\subsection{Substrate preparation}

The samples were prepared on $2.2 \mathrm{~mm}$ thick glass substrates, coated on one side with a $300 \mathrm{~nm}$ transparent conductive layer of fluorine tin oxide (FTO). The substrates were purchased from Solaronix SA (Catalogue No. TCO22-15 43273).

A crucial requirement for the metal replication is the continuity of the gyroid morphology at the bottom and top interfaces. In thin films, the continuity can be affected by asymmetric interaction energies with free or confining interfaces that can lead to surface reconstruction morphologies ??. Depending on the affinity between the substrate and each of the blocks, one the polymers might be favoured to contact the substrate, resulting in the formation of a thin continuous layer underneath. To prevent such a reconstruction, the substrate was functionalized 
to increase the hydrophobicity by a procedure optimized for each substrate-block copolymer combination.

The FTO glass was initially cleaned for 20 minutes at $80^{\circ} \mathrm{C}$ in a piranha solution formed by three parts in volume of sulfuric acid and one part of hydrogen peroxide. The substrates were then rinsed and sonicated for 10 minutes with deionised water to remove any residual piranha solution. They were then dried with a nitrogen gun and placed on a hotplate at $60^{\circ} \mathrm{C}$ for 10 minutes to let the water evaporate. The substrates were then functionalized with silane to create a hydrophobic surface. In the case of ISO triblock copolymers, the silane functionalization was optimized at 20 seconds immersion in a solution of $0.2 \% \mathrm{vol}$ of octyltrichlorosilane in cyclohexane, followed by nitrogen flow drying.

\section{$3.3 \quad$ I-S-O triblock copolymer}

The triblock copolymers used in this project were synthesized and supplied by Wiesners group from Cornell University. They are terpolymers composed of polyisoprene (PI), polystyrene (PS) and polyethylene oxide (PEO), commonly named ISO block copolymer. The volume fraction of the three blocks was, respectively, $31 \%, 53 \%$ and $16 \%$ s adopting the double gyroid morphology at equilibrium, with PI and PEO as the two minor single-gyroid phases embedded in the majority PS phase.

Two different batches were supplied with total molecular weights of 33 and $53 \mathrm{~kg} \cdot \mathrm{mol}^{-1}$ corresponding to measured unit cell sizes of 35 and $50 \mathrm{~nm}$, respectively. The block copolymer were supplied as a powder in an amorphous state.

After the annealing and the self-assembly the PI phase was selectively removed by UV radiation (at $254 \mathrm{~nm}$ for 2 hours) to break the carbon-carbon double bonds of the polyisoprene. The film was then immersed in ethanol to dissolve the cleaved polymer. While breaking the PI bonds the UV light induced also the cross-linking of the PS. After the metal electrodeposition the PS and PEO were removed by plasma etching. 


\subsubsection{Thermal annealing}

A solution of $10 \mathrm{wt} \%$ of ISO block copolymer in anisole was spin coated onto a FTO glass substrate to produce a film of about $1 \mu \mathrm{m}$ thickness. During the spincoating, the solvent was rapidly removed by centrifugal expulsion, viscous outflow and evaporation, leaving the block copolymer in a meta-stable amorphous state with no ordered phase separation.

Despite of the low affinity of the different polymers their mobility was not high enough to lead to the self-assembly at room temperature. To promote the self-assembly to the more energetically favourable gyroid phase the mobility of the polymer chains could be increased thermally.

The glass transitions of the polyisoprene and polyethylene oxide homopolymers are $-70^{\circ} \mathrm{C}$ and $-54^{\circ} \mathrm{C}$ respectively, whereas that of the polystyrene homopolymer is around $100^{\circ} \mathrm{C}$. Therefore, above this temperature the block copolymer started behaving as a melt. The most effective thermal annealing resulted to be in vacuum at $180^{\circ} \mathrm{C}$ for 30 minutes with heating rate of $150^{\circ} \mathrm{C} / \mathrm{h}$ followed by slow cooling in vacuum. The heating rate did not influence the process significantly whereas the cooling rate influenced the top surface reconstruction and the presence of the polyisoprene block at the top interface.

The thermal annealing produced very uniform samples with high reproducibility, but the size of the gyroid domains was limited to few a microns which represented a drawback for some optical characterization methods. The origin of the domain size can be identified considering the self-assembly mechanism. As the chain mobility is increased thermally, the amorphous film starts to self-assemble into the gyroid phase by a mechanism resembling nucleation and growth ?. This process starts simultaneously across the entire film at distances on the same order of magnitude of the final size of the domains and terminates when the domain boundaries of the gyroid phases reach each other. A typical scale of the domains formed by thermal annealing are shown if Figure 3.2. The increase of the domain size has been attempted by slowing down the heating rate in order to promote the growth process respect to the nucleations. No sensible domain increase was found and below $20^{\circ} \mathrm{C} / \mathrm{min}$ the long times at high temperature caused the degradation of the polymers. 


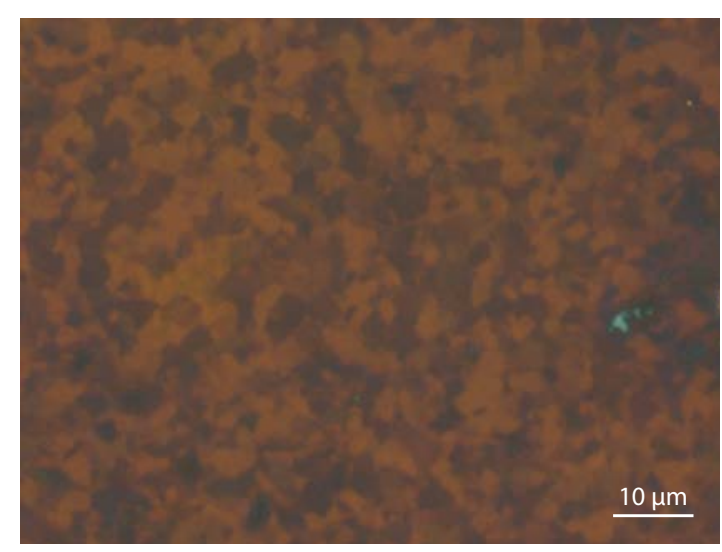

Figure 3.2: Domain morphology of gyroids obtained by thermal annealing. The image is obtained after the metal replication by optical microscopy and under linear polarized light. As discussed in the next chapter, the metal gyroids show a strong linear dichroism that enables the inspection of the domain size by optical microscopy.

For the optical properties discussed in the next chapters it is important to highlight that all ISO gyroid domains in thin films had a [110] out of plane orientation and random in-plane orientations (Figure 3.3). This uniaxial orientation stems from the interaction of the block with the upper air surface of the film where nucleation starts in preferred orientation. Whether gyroid nucleation in thin films starts at the top or the bottom interface has been object of interest for a long time, because of the aim to induce domain ordering by substrate patterning ?.

To investigate the nucleation mechanism, the thin film was quenched during thermal annealing at an initial stage of the self-assembly process. The cross section of the film displayed in Figure 3.4 clearly shows that the block copolymer had self-assembled only in the top part of the film, demonstrating that the selfassembly of the ISO block copolymer was nucleated at the top surface.

\subsubsection{Drying-annealing}

The first gold gyroid metamaterials were produced in 2009 by Nataliys Yufa by drying annealing. A solution of $10 \mathrm{wt} \%$ of ISO block copolymer in anisole was 

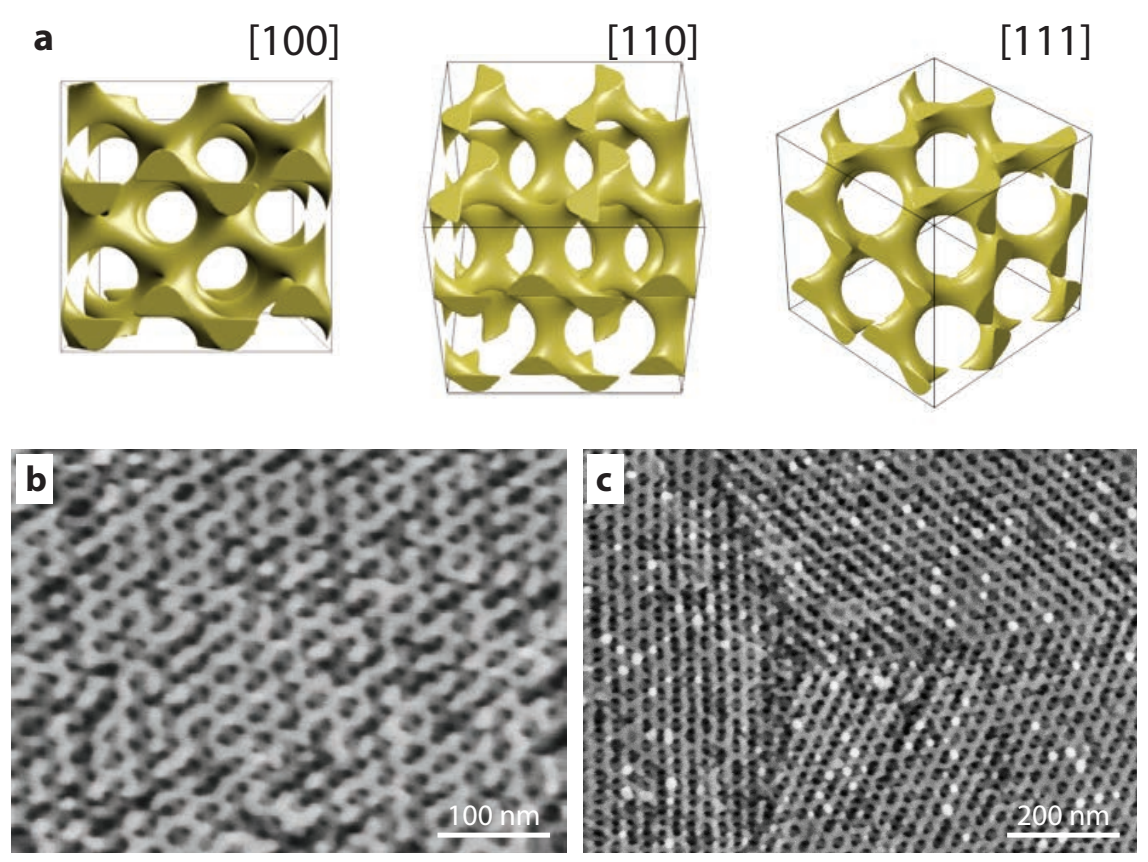

Figure 3.3: (a) Schematic of the gyroid structure as seen from the indicated crystal directions. The [110] direction in the ISO triblock copolymer is always perpendicular to the surface of the gyroid samples as shown in (b), while the in plane orientation is completely random (c).
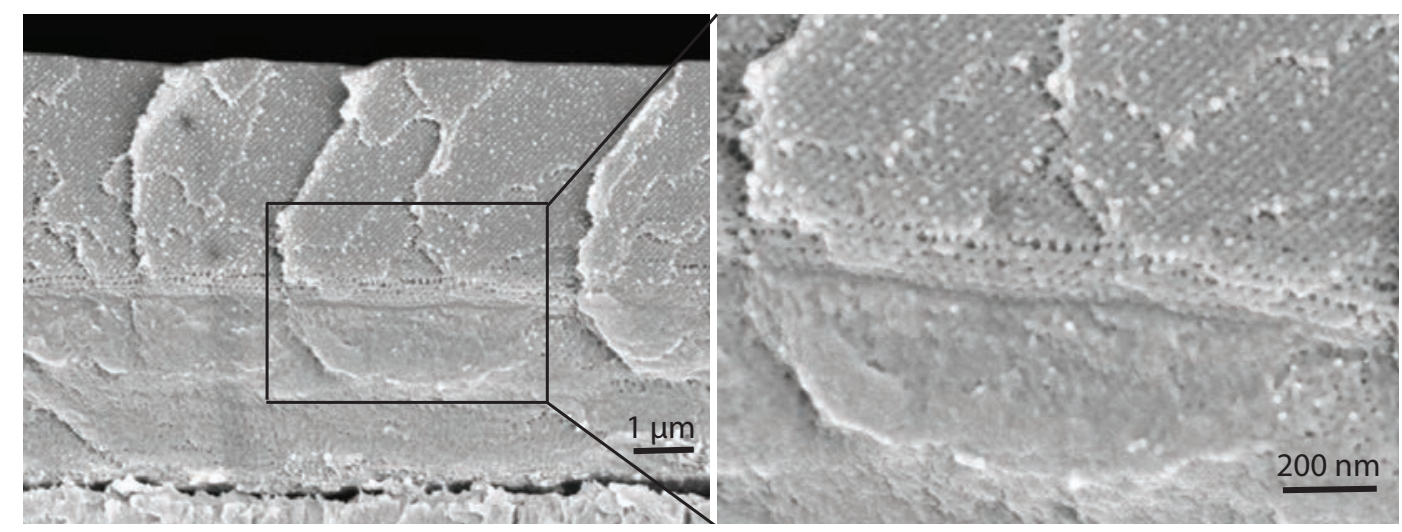

Figure 3.4: Scanning electron microscope (SEM) image of the gyroid block copolymer cross section at the half-stage of the thermal annealing. The ordered structure at the top portion of the film suggest that the annealing process is initiated at the top interface. 
blade coated onto the FTO glass and covered with a glass dome to slow down the solvent evaporation. The glass substrates were not functionalized and cleaned by acetone and isopropanol rinsing. Although this method produced some good samples for the first characterization, it lacked reproducibility.

The procedure I developed allowed controlled dry annealing on a functionalized substrate. Normally, the drying annealing procedure is not suitable for hydrophobic substrate as the blade coated solution dewets, preventing thin film formation. On the other hand, a very concentrated and viscous solution cannot be used in blade coating. Nonetheless, a concentrated and viscous solution could be deposited onto the functionalized substrate by spin coating for very short times, forming a film. During the spin-coating most of the solution is rapidly removed by an initial centrifugal expulsion, followed by viscous outflow and finally by solvent evaporation. By spin coating the solution of $10 \mathrm{wt} \%$ ISO block copolymer in anisole at 1200rpm for only 8-10 seconds the centrifugal expulsion and viscous outflow are completed while only partial evaporation takes place, giving rise to a stable viscous thin film stable on the hydrophobic substrate.

During the drying annealing the polymers are initially dispersed in the solvent with high mobility and a reduced chain interaction. As the solvent slowly evaporates, the concentration of the solution increases. At a certain concentration the the polymers, while still having some chain mobility, start microphase-separate into the gyroid morphology. As the solvent initially evaporates faster at the edges, leading to a drying front that propagates towards the centre of the film, this mechanism induces the self-assembly by a slow continuous process, which is different from nucleation and growth during thermal annealing. For this reason, by drying-annealing it was possible obtain very large domains, on the order of hundreds microns as shown in Figure 3.5.

\subsubsection{Solvent vapour annealing}

A more conventional solvent vapour annealing procedure was also investigated. The ISO block copolymer thin film was placed into a chamber with a solvent vapour control. The thin film interference of the block copolymers was monitored by a double fibre setup that allowed precise measurement of the film thickness 


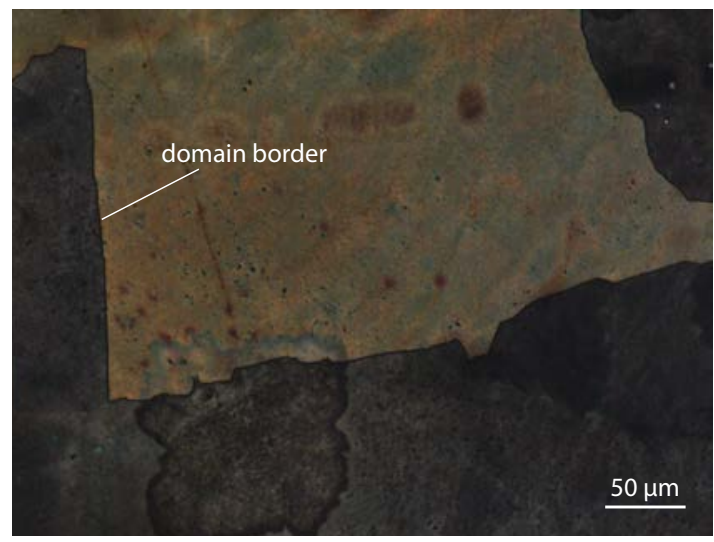

Figure 3.5: Domain morphology of gyroids obtained by drying-annealing. The image was obtained by optical microscopy under linear polarized light after metal replication. As discussed in the next chapter, the metal gyroids show a strong linear dichroism that enables the inspection of the domain size by optical microscopy.

related to the partial vapour pressure of the solvent. The anisole vapour pressure was increased to saturation and then slowly reduced to promote the gyroid selfassembly in a similar way as drying-annealing. Some successful gyroid formation was obtained for a decrease in solvent concentration below $4 \% / \mathrm{h}$. However, the dimension of the domains of the gyroid obtained by solvent annealing were on the order of 5 to 10 microns, significantly smaller than those obtained by dryingannealing.

\subsection{PFS- $b$-PLA diblock copolymer}

Double gyroids were produced from a Poly(4-fluorostyrene)-block-poly(D,L-lactide) diblock copolymer (PFS- $b$-PLA, $M_{\mathrm{w}}=24 \mathrm{~kg} \cdot \mathrm{mol}^{-1}$ with $38 \mathrm{vol} \%$ PLA fraction) synthesized by Maik Scherer by atom transfer radical polymerization ?, followed by the addition of lactide via organocatalytic ring-opening polymerization?.

In the PFS- $b$-PLA diblock copolymer the polylactide (PLA) formed two distinct gyroid networks embedded in the polyfluorostyrene (PFS) major phase. The thermal annealing procedure was developed by Maik Scherer. The polymer films 
were prepared by spin coating a $10 \mathrm{wt} \%$ toluene solution onto silanized FTO-glass substrates. The films were heated at $175^{\circ} \mathrm{C}$ under a nitrogen atmosphere for 20 minutes at a ramping rate of $150{ }^{\circ} \mathrm{C} /$ hour. After quenching to room temperature, the films were soaked in $0.3 \mathrm{M} \mathrm{NaOH}$ solution containing 1:1 (wt) mixture of water and methanol for one hour to cleave the PLA phase ??. After electrodeposition the remaining polystyrene (PFS) majority phase was finally dissolved in toluene.

Unlike the ISO triblock copolymer the PFS- $b$-PLA diblock copolymer produces a double gyroid with random orientation both in the plane and out of plane directions. The size of the gyroid domains was only 1-2 microns after thermal annealing. In order to produce larger domains, drying-annealing was attempted using a similar procedure as for the ISO block copolymer. Unfortunately film dewetting occurred even at high solution concentrations, preventing the formation of large domains of the double gyroid.

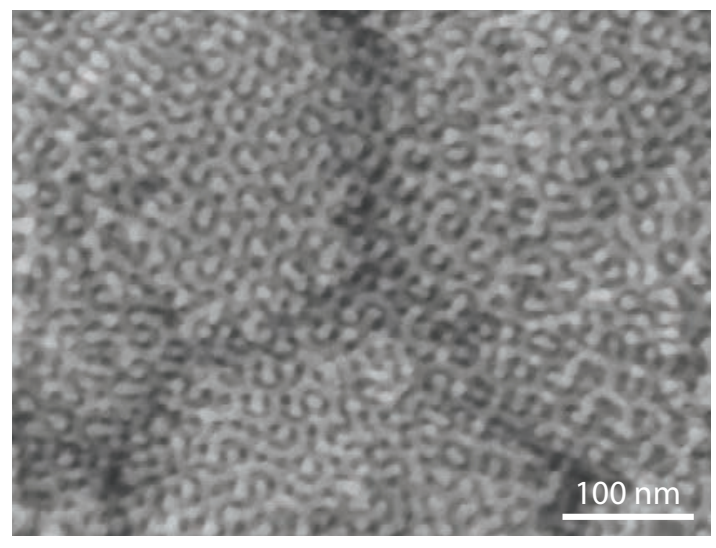

Figure 3.6: SEM image of the gold double gyroid produced from the PS-PLA diblock copolymer.

\subsection{Metal deposition}

After gyroid minor phase removal, the block copolymer templates were backfilled by metal electroplating. In an electroplating process the electric current reduces the metal cations dissolved in the electrolyte to metal, therby creating a deposit 
on the working electrode (i.e. the FTO layer). The electrochemical setup was composed of an FTO layer acting as working electrode, a saturated calomel electrode as reference electrode, and a platinum mesh as counter electrode. The electrodes were immersed in a liquid electrolyte containing the metal ions to be deposited. The three-electrode cell was controlled by a PGSTAT302N potentiostat and used in potentiostatic mode, holding the voltage between the working and the counter electrode at a defined value.

Metal electroplating follows a mechanism of nucleation and growth from the working electrode. To form continuous gyroid films within the block copolymer templates it was crucial to promote nucleation over the growth process. This was accomplished by cyclic voltammetry, sweeping the voltage from 0 to an overpotential that induced nucleation, and back to 0 again. The voltage was then set to a constant value for a time varied by the desired final thickness.

For gold deposition, 0.5 vol\% brightener was added to the plating solution (Technic Inc., ECF60) to achieve a smoother deposition. The initial cyclic voltammetry ranged from $0 \mathrm{~V}$ to $-1.2 \mathrm{~V}$ to $0 \mathrm{~V}$ at $50 \mathrm{mV} / \mathrm{sec}$. The steady potential was set at $-0.8 \mathrm{~V}$.

For the nickel deposition, the plating solution was purchased from Alfa Aeser, the cyclic voltammetry voltage range was $-1.4 \mathrm{~V}$ at $70 \mathrm{mV} / \mathrm{sec}$ and the steady potential was $-1.1 \mathrm{~V}$

The silver plating solution was purchased from Metalor (Metsil $500 \mathrm{CNF}$ ), the cyclic voltammetry swept down to $-1 \mathrm{~V}$ and the steady potential was held at $-0.7 \mathrm{~V}$.

\subsection{Film morphology control}

The thickness of the metal gyroid films produced by electrodeposition varied across the film due to the roughness of the FTO layer and to the fluctuations of the deposition rate. The resulting roughness was on the order of $\pm 50 \mathrm{~nm}$ both at the top and bottom interfaces as shown in Figure 3.7(a-e). As discussed in the next chapter, some optical characterizations required gyroid films with very precise film thickness control, which stimulated the development of a new fabrication procedure. 
The roughness of the bottom interface was reduced using glass coated with Indium Tin Oxide (ITO) as transparent conducting substrate. The FTO substrates are commonly preferred to ITO as the roughness is known to reduce the planar interface reconstruction of block copolymer self-assembly?. Gold was deposited into the voided self-assembeld ISO gyroid channels from the flat ITO glass (Figure $3.7(\mathrm{c})$ ) by unctionalizing the substate by immersion in a solution of $0.5 \mathrm{vol} \%$ octyltrichlorosilane in cyclohexane for 30 seconds.

The roughness control at the top surface was achieved by developing a fabrication method termed overgrowth-removal. The metal was electrodeposited through the entire voided block copolymer template to form a thick overgrowth layer of bulk metal on top of the film. The continuous layer of bulk metal was then peeled off from the block copolymer template or cleaved by sonication, creating a smooth, planar surfaces of the metal electrodeposited, replicating the exact surface smoothness of the block copolymer film produced by spin coating (Figure $3.7(\mathrm{~b}-\mathrm{d}-\mathrm{f}))$.

Those fabrication methods could also be extended to produce gyroid films with design topography patterns, adding an extra degree of freedom to the design of the metamaterial structure. The proof of concept was realized by replicating a grating pattern into the gyroid film via nanoimprinting?. An optical grating was used as master and replicated in a polytetrafluoroethylene (PTFE) film at $230{ }^{\circ} \mathrm{C}$ and 40 bar. The patterned PTFE film was then used as master to imprint into the block copolymer film during the thermal annealing by applying a pressure of 20 bar. Finally, the metal replication of the block copolymer template successfully replicated both the gyroid morphology within the films and the surface topography of the grating as shown in Figure 3.8.

\subsection{Conclusions}

The good reproducibility achieved with the fabrication of gyroid metamaterial is an essential requirement for a consistent study of their optical properties. Moreover, it underlines the potential of the bottom-up approach by block copolymer self-assembly in producing a truly three dimensional optical metamaterial. In the following chapters I present the optical properties of this gyroid metamaterials 

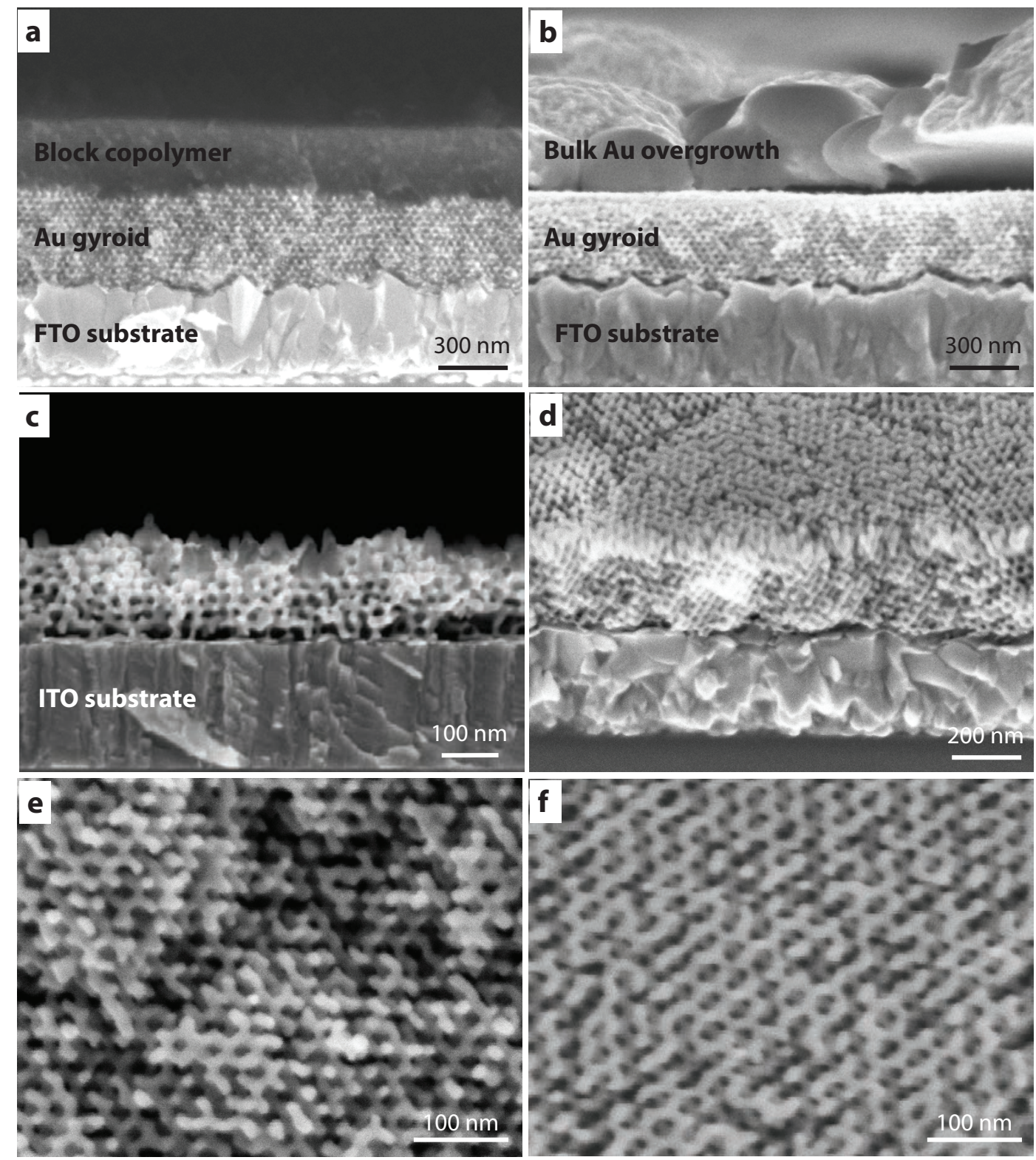

Figure 3.7: (a) SEM image of the film cross-section revealing the roughness of the FTO substrate and of the gold gyroid electrodeposited into the block copolymer template. (b) SEM cross-sectional image of the metal gyroid produced by overgrowth removal. The thick layer of overgrowth gold can be detached from the film producing a flat surface of the metal gyroid. (c) SEM cross-sectional image of the gyroid produced on flat ITO substrate. (d) SEM image at $45^{\circ}$ of gyroid produced on flat ITO substrate and by overgrowth removal. (e-f) SEM images in top view of the metal gyroid films produced without and with morphology control. 

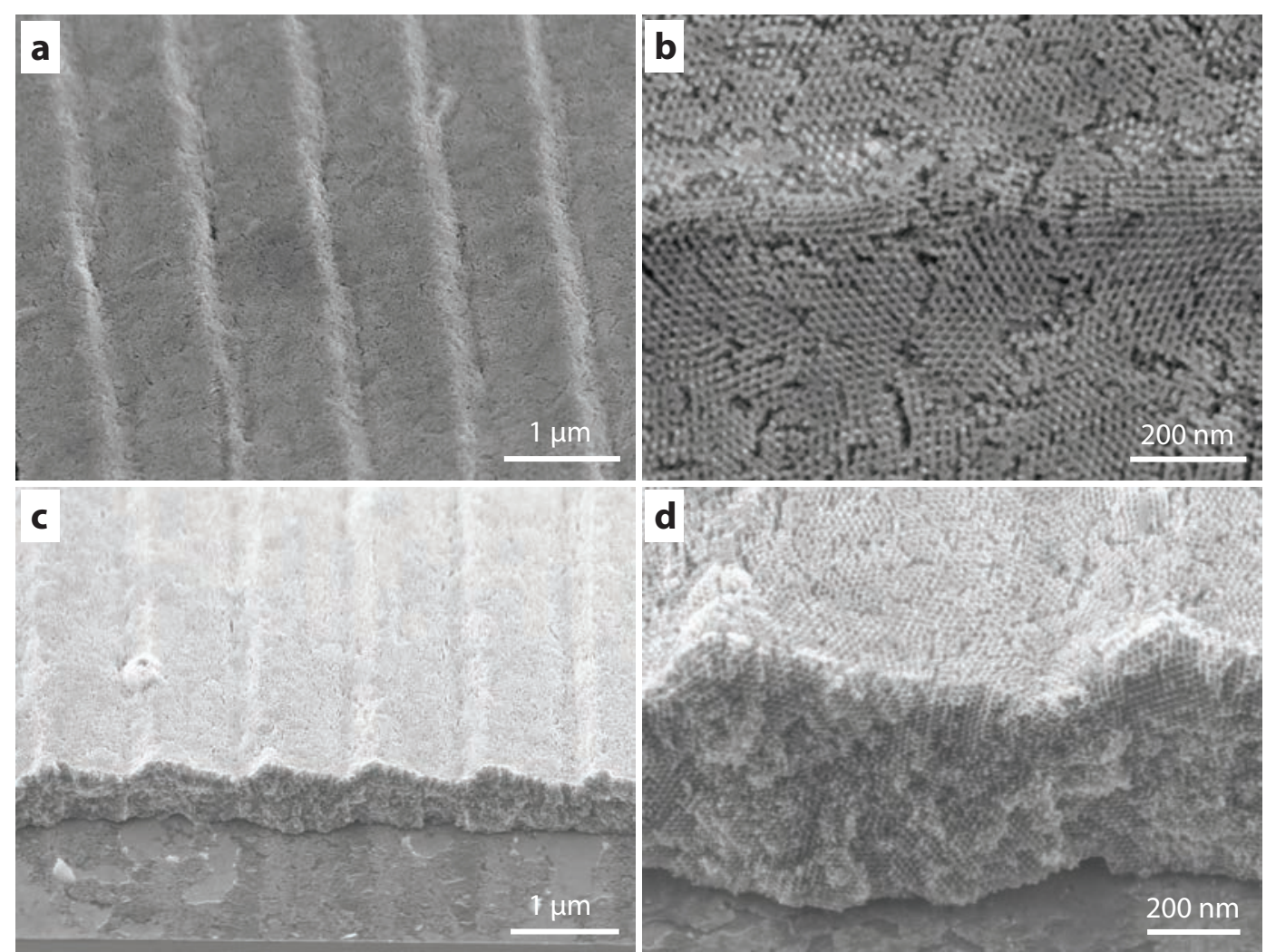

Figure 3.8: SEM images of the metal gyroid with grating patterned topography produced by the overgrowth-removal process. 
and some further developments in the fabrication process. 


\section{Chapter 4}

\section{Gyroid metamaterial characterization}

\subsection{Introduction}

In this chapter I will present full characterization of gyroid metamaterials.

The atomic structure of the gold gyroid was investigated using high resolution by transmission electron microscopy (TEM), revealing the small size of the metal grains and a very smooth surface of the gyroid struts.

The electrical characterization will then be discussed comparing the resistivity of the gyroid gold with the bulk gold.

Subsequently, an extensive section will be dedicated to the optical properties, studied by reflection and transmission analysis. A thin wire array model will be discussed to understand the origin of the optical response. Simulation spectra were produced in collaborations with two theoretical groups and will be presented together with my experimental results.

The larger part of the gyroid metamaterial investigation was conducted on a single gold gyroid, referred to simply as "gold gyroid", with a unit cell size of $35 \mathrm{~nm}$ and domains with the [110] orientation out of plane.

Finally, in the last part of the chapter I will discuss the optical properties of silver and double gyroids. 


\subsection{Transmission electron microscopy}

The atomic structure of the gold gyroid was investigated by high resolution by transmission electron microscopy (TEM) [?] in collaboration with Dr. Caterina Ducati $^{1}$. As depicted in Figure 4.1 the gold atoms show crystalline structure with no lattice vacancies and the size of the grains is on the order of a few tens of nanometres. The dimension of the grains was influenced by the electrodeposition through the nanosized network template that produces the formation of new grains at each struts intersection. The surface roughness of the gyroid struts is remarkably small, with imperfections only at the atomic scale.

Energy dispersive X ray diffraction did not reveal any other element in addition to gold, excluding contributions of impurities in the optical effects.
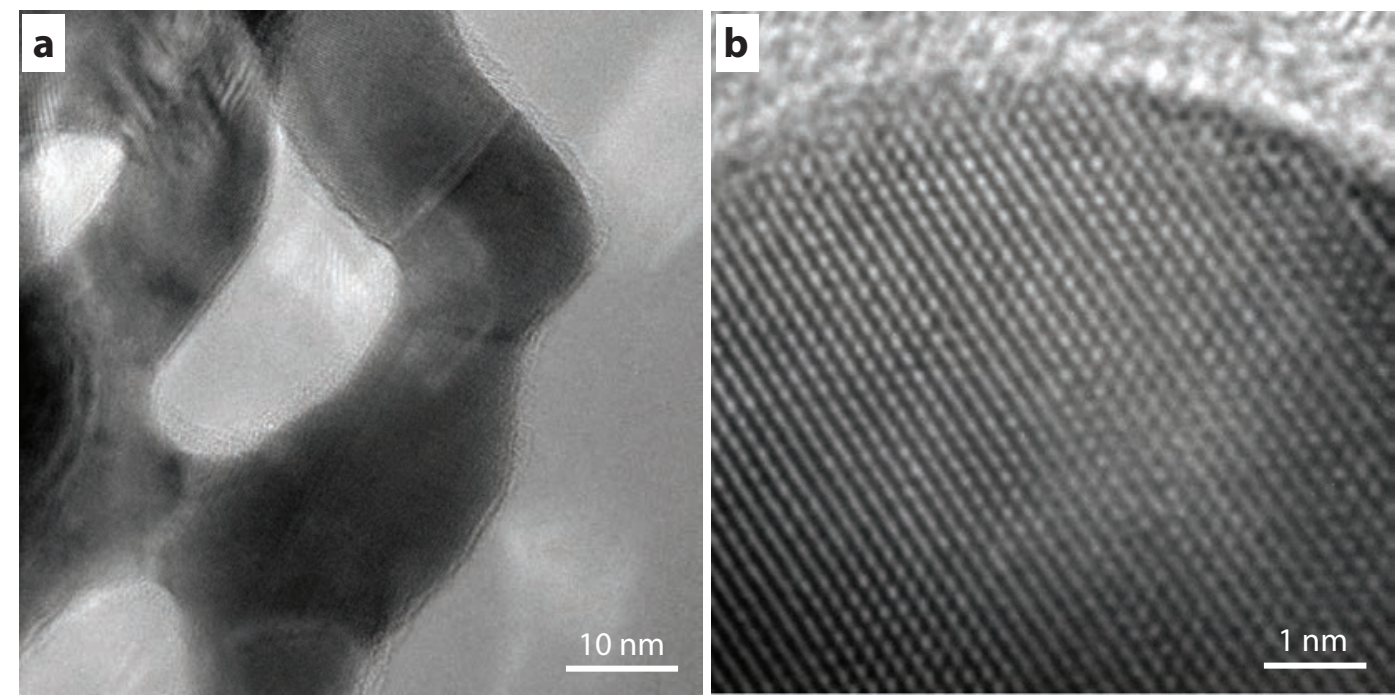

Figure 4.1: Transmission electron microscopy (TEM) images of the gold gyroid. Different shades of gray in (a) represent the gold grains. (b) High resolution detail of a gyroid strut showing the metal crystallinity and very smooth surface.

\footnotetext{
${ }^{1}$ Electron Microscopy Group, Department of Materials Science and Metallurgy, University of Cambridge
} 


\subsection{Electric characterization}

The nanoscale cross-section of the gyroid struts and the small dimension of the gold grains produced a significant increase of the electric resistivity as revealed by four probe measurements.

In the four probes setup, the probes were placed in one line, in contact with the gyroid film and a current was supplied to the two outer probes. The resistance of the gyroid film was extracted by the voltage drop between the two inner probes taking into account the geometrical factors of the probes. The resistivity of the supporting FTO layer from the product specifications was taken into account as a parallel resistor.

The four probe measurements yields the sheet resistance $R_{s}$, commonly defined as the resistance per aspect ratio. The intrinsic material resistivity $\rho$ was then extracted by multiplying the $R_{s}$ by the film thickness and the gold filling fraction. The measurement was performed on films with different thicknesses to verify the consistency of the measured values. The results are presented in Figure 4.2 and revealed an increase of resistivity to $\rho_{\text {gyr }} \approx 52 \mu \Omega \cdot \mathrm{cm}$, about 20 times higher than of bulk gold $\rho_{\text {bulk }} \approx 2.44 \mu \Omega \cdot \mathrm{cm} ?$.

Two contributions are responsible for the increase in the electric resistivity of gold in the gyroid architecture, the grain boundaries and surface scattering. From thin wire studies [?] it is know that for wires widths below approximately 0,5 times the mean grain size, surface scattering becomes important, approaching the same order of magnitude of grain-boundary scattering as the width decreases. Surface scattering and grain boundary imposes restrictions on the electron mean free path, which is $40 \mathrm{~nm}$ in gold [?].

As revealed by the TEM analysis, the grain size in the gold gyroid was $20-30$ $\mathrm{nm}$ and the strut widthv was $\sim 10 \mathrm{~nm}$. It is therefore reasonable to conclude that both grain boundary scattering and surface scattering have similar contributions to the resistivity.

Silver gyroids were also characterized electrically, resulting in a $\rho_{\mathrm{gyr}} \approx 40 \mu \Omega \cdot \mathrm{cm}$. They had resistivities which were about 25 times higher than the bulk silver $\rho_{\mathrm{bulk}}=1.59 \mu \Omega \cdot \mathrm{cm}$. 


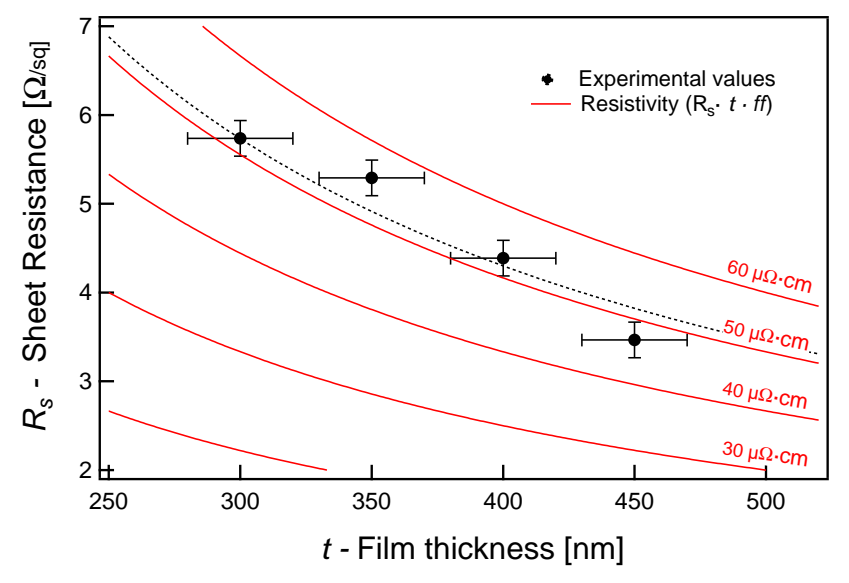

Figure 4.2: Resistivity measurements for gold gyroid. The sheet resistance $R_{s}$ of the gyroid film was measured by a four probe setup and multiplied by the film thickness and the filling fraction to extract the resistivity of gold in the nanostructured gyroid architecture. The values measured for different thicknesses reveal an average resistivity of $\sim 52 \mu \Omega \cdot \mathrm{cm}$, about 20 times higher than the value of $2.44 \mu \Omega \cdot c m$ for bulk gold. The red lines illustrate the variations of the resistivity in function of film thickness and sheet resistance.

\subsection{Optical properties}

\subsubsection{Single gyroid}

The optical response of the gold gyroid differs significantly from normal gold, exhibiting a red hue in reflection and a finite transmission (Figure 4.3(a-b)).

The samples were characterized spectroscopically using an optical microscope with white light illumination and fiber detection in a confocal configuration. Confocal optical microscopy was performed using a $50 \mathrm{~m}$ core optical fiber that served as pinhole in the conjugate to the focal plane of a $\times 20$ microscope objective. A broad-band halogen lamp acted as illumination source. Linear polarization measurements were obtained using achromatic polarizers (Thorlabs Inc.) and for circular polarization measurements the polarizers were combined with superachromatic quarter wave quartz plates (B. Halle Nachfl. GmbH). Reflection measurements were normalized to a silver mirror reference, whereas transmission measurements were normalized to the FTO-glass substrate. 


\section{Gyroid metamaterial characterization}

The experimental results for both reflection and transmission configurations are compared in Figure 4.3(c) with the reflection of bulk gold produced by electrodeposition. The reflectivity of the gold gyroid is characterized by a dip at around $520 \mathrm{~nm}$ and a steady increase towards longer wavelengths.

A remarkable transmission of $20 \%$ was found for a $300 \mathrm{~nm}$ thick layer with the smaller gyroid unit cell. This high transmission across a several-hundrednanometer-thick layer with $\mathrm{Au}$ volume fractions of $30 \%$ is evidence for the transport of the optical energy flux through the strut network by plasmon resonances which is the hallmark of an optical metamaterial.
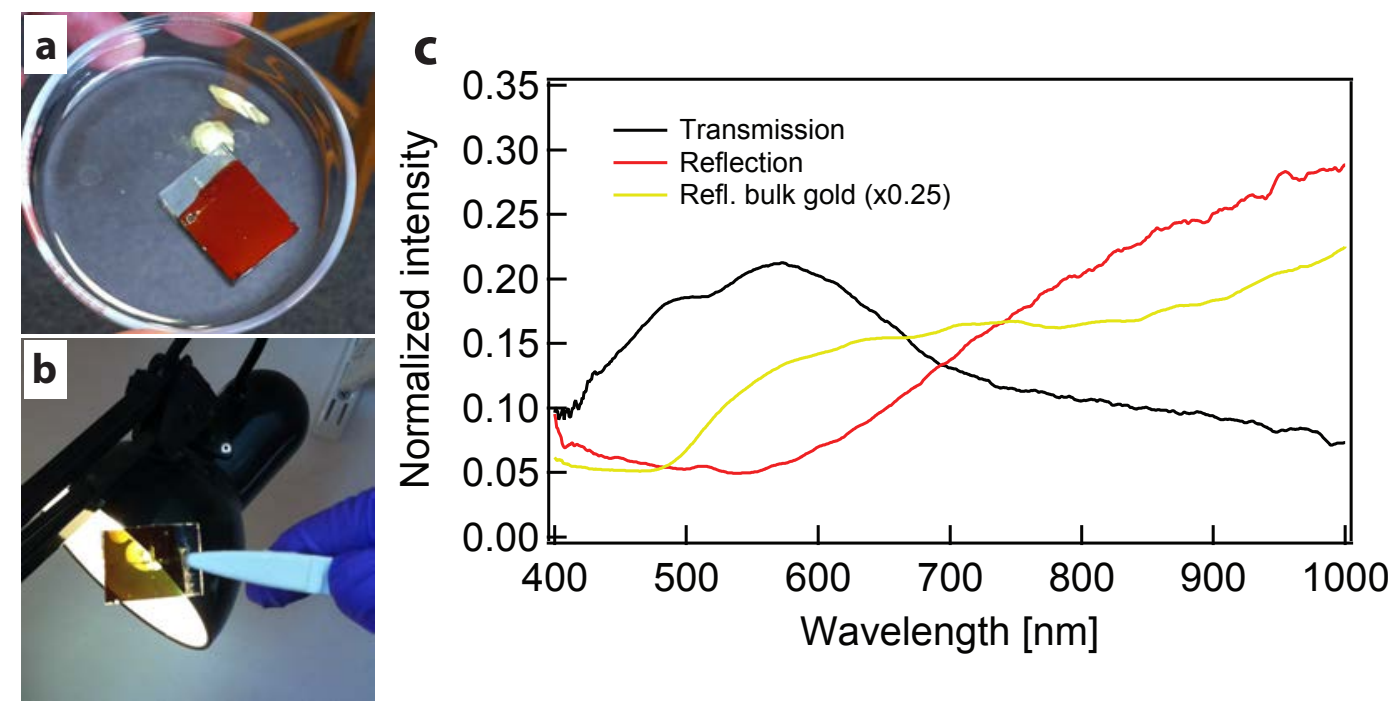

Figure 4.3: (a-b) Appearance of the gold gyroid in reflection and transmission. (c) Transmission and reflection spectra for unpolarised incident light compared to bulk gold. Film thickness $\sim 300 \mathrm{~nm}$.

Thin wires model The optical properties of the gold gyroid can be understood considering in first approximation the gyroid in a simplified wire array model. In 1998 J. Pendry [?] showed that light interacting with an extended 3D network of thin wires (Figure 4.4) induces a magnetic field that impedes the free electronic motion, producing an increase in the electron effective mass:

$$
m_{\mathrm{eff}}=\frac{\mu_{0} e^{2} \pi r^{2} n}{2 \pi} \ln (a / r)
$$




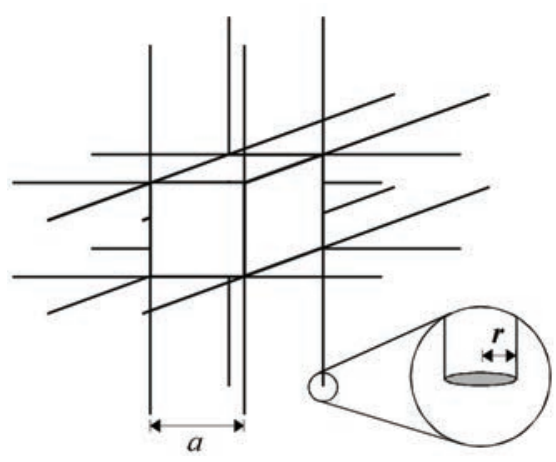

Figure 4.4: Periodic structure model composed of infinite wires arranged in a cubic lattice. Adapted from [?].

where $\mu_{0}$ is the permeability of free space, $e$ the electron charge, $n$ is the density of electrons in the wires, $r$ the radius and $a$ the cell side of the square lattice of the wires.

The response of a material to electromagnetic radiation can be described by its dielectric function that relates the applied electric field to the local field created inside the material. In the Drude model, the dielectric function is derived only in terms of the response of the free electrons. These can be considered as classic oscillators with a characteristic oscillation frequency called the plasma frequency. The increase of the electron effective mass in the thin wire network modifies the plasma frequency and the damping of the electron motion by the relations:

$$
\begin{gathered}
\omega_{\mathrm{eff}}^{2}=\frac{n_{\mathrm{eff}} e^{2}}{\epsilon_{0} m_{\mathrm{eff}}} \\
\Gamma_{\mathrm{eff}}=\frac{\epsilon_{0} \omega_{\mathrm{eff}}^{2} \rho}{f},
\end{gathered}
$$

where $n_{\text {eff }}=f n$ is the effective concentration of the metal free electrons modified by the filling fraction $f, \epsilon_{0}$ is the vacuum permittivity, and $\rho$ the resistivity of gold.

Therefore, the gyroid structure modifies the plasma frequency and the dielectric function of gold, effectively creating a new metal with its own optical properties. 


\section{Gyroid metamaterial characterization}

The reflectivity of the gold gyroid arising from the effective dielectric function was simulated by Petros Farah ${ }^{1}$ in transfer-matrix method simulations, modifying the Drude model to take into account the gold interband transitions, including the experimental value of the gold resistivity in the gyroid. The plasma frequency reduction was estimated from the bulk value of $7.5 \mathrm{eV}$ to $3.5 \mathrm{eV}$, in good agreement to the experimental results.

FDTD calculations Although the thin wire model was able to predict the reflection response of the gold gyroid in terms of the reduced plasma frequency and increased damping, it was unable to describe the propagation of the plasmonic modes in the gyroid. As a consequence, the transmission properties were not correctly described.

The simulation of the field enhancement and its propagation in the gold gyroid was developed by Sang Soon $\mathrm{Oh}^{2}$ [?] by finite different time domain (FDTD) calculations [?], shown in Figure 4.5. Both the electric and magnetic fields are less attenuated around $550 \mathrm{~nm}$, in good agreement with the experimental transmission peak. The predicted magnetic field has a modest spacial variation around the network. In contrast, the electric field distribution is enhanced in the proximity of the gold struts. The absence of narrow peaks in the reflection and transmission spectra was an indication for the absence of localized modes. Nonetheless the FDTD calculations display the excitation of some weak localized surface plasmons.

Linear and circular dichroism When illuminated under linearly polarized light the gold gyroid show strong birefringence. This effect is shown in Figure 5.6 where two gyroid domains have in-plane orientation orthogonal to each other. Under unpolarized light illumination (Figure 5.6(a)) the different domains are not distinguishable, whereas when the polarization of the incident light is parallel to the [110] in-plane direction of one of the areas and nearly perpendicular to the [110] in-plane direction of the neighbour, they show different optical responses.

This birefringence arises from the relative gyroid orientation and the polariza-

\footnotetext{
${ }^{1}$ Nanophotonics group, University of Cambridge

${ }^{2}$ Ortwin Hess' group, Department of Physics, Imperial College London
} 


\section{Gyroid metamaterial characterization}

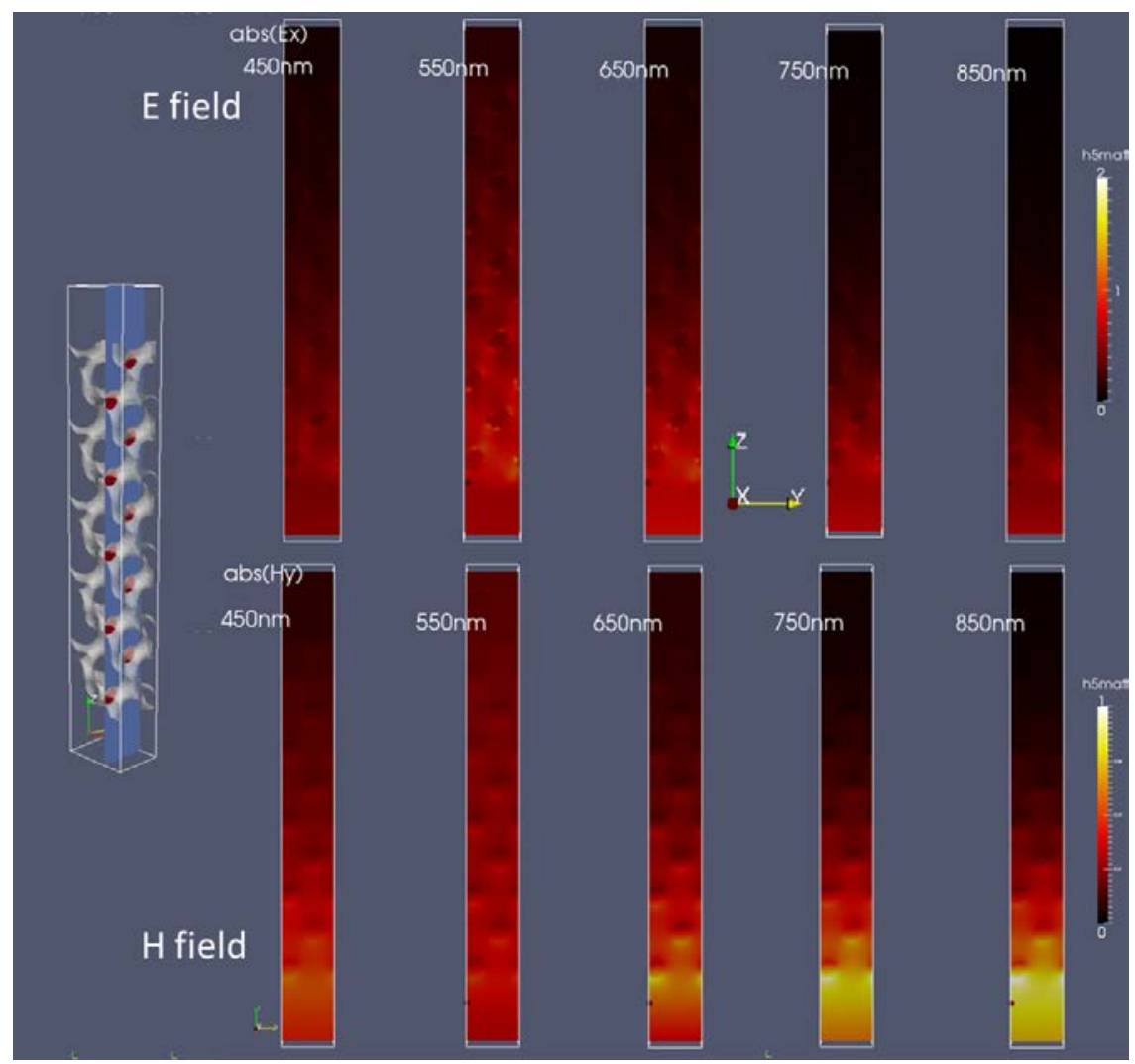

Figure 4.5: FDTD calculation of the electric and magnetic field distributions in the metal gyroid for continuous wave excitation linearly polarized in the $x$ direction. The magnetic field has a modest spacial variation around the gyroid, whereas the electric field distrubution is enhanced in proximity of the gyroid struts. Courtesy of Sang Soon Oh.

tion direction, where light is coupled differently to localized plasmon resonances. In Figure 5.6(d) the variation of reflectivity is plotted as a function of the angle between the in-plane [110] gyroid direction and the incident light polarization. Stronger reflectivity was found for linear polarization perpendicular to the [110] in-plane orientation.

The gyrotropic behavior of the gyroid metamaterial was probed in a previous study by Silvia Vignolini [?] by transmission measurements with left- and right-hand circularly polarized light. Figure 4.7 plots the gyrotropic tranmission as difference between the two circular polarizations, averaged over frequency re- 

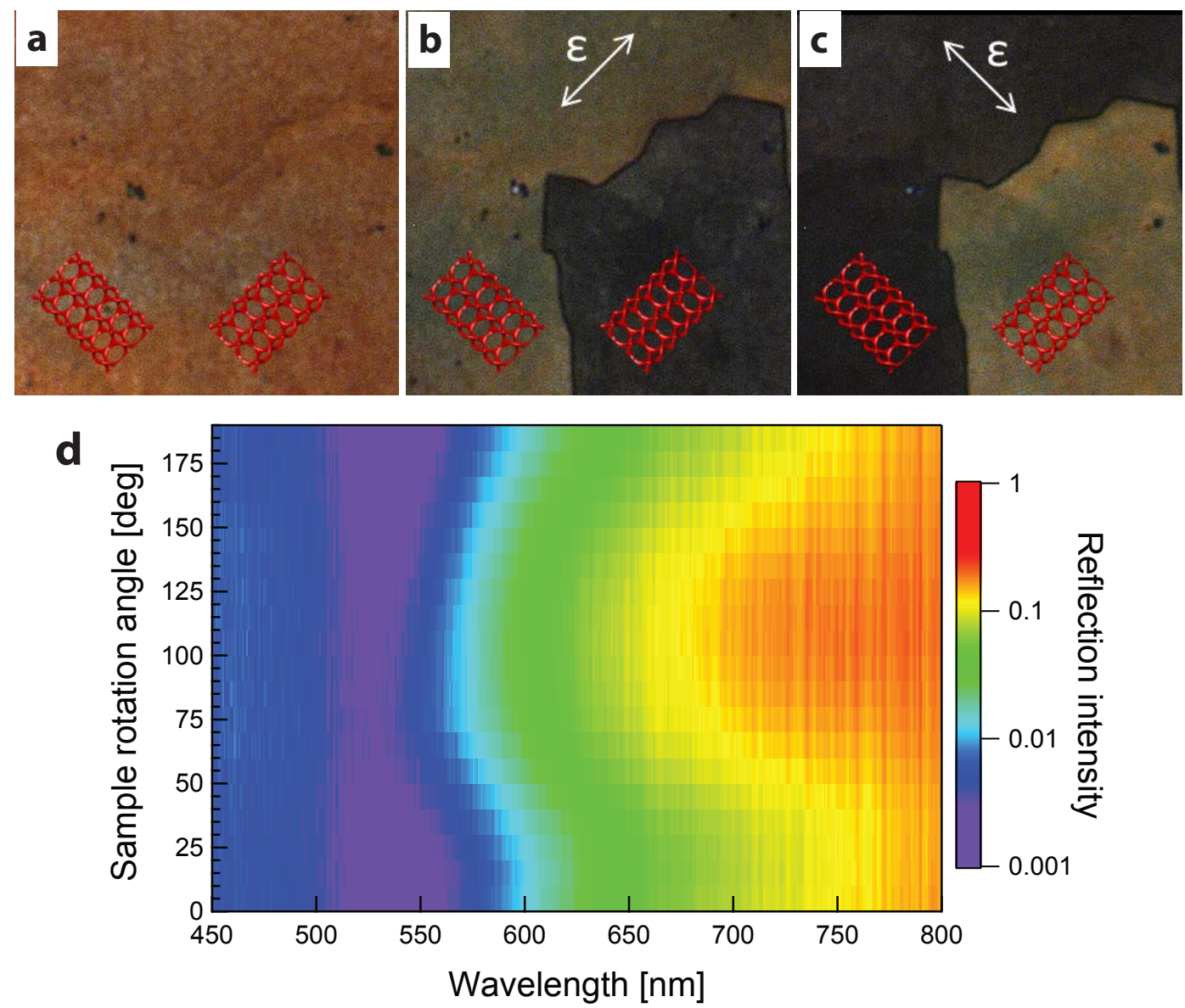

Figure 4.6: (a) Optical reflection images for unpolarized light. (b-c) Optical reflection images obtained with linearly polarized illumination. The relative orientation of the gyroid domains (red inserts) and the light polarization (the white arrows) produces a strong birefringence effect. (d) Variation of the reflection spectra as a function of the angle between the in-plane [110] gyroid direction and the incident light polarization.

gion between 600 and $750 \mathrm{~nm}$, for different gyroid orientations. As expected, the strongest gyrotropic effect was observed along the chiral [111] direction. As discussed in the previous chapter, the samples produced by the triblock copolymer gyroid exhibit constant [110] orientation in the out-of-plane direction. To access the [111] direction the sample was tilted by $35^{\circ}$ and rotated around the [110] surface normal. 


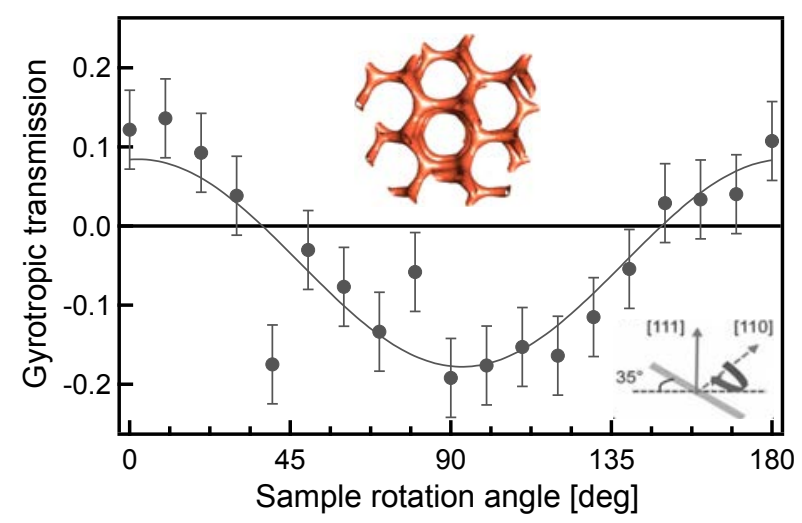

Figure 4.7: Gyrotropic behaviour of the gold gyroid with a $50 \mathrm{~nm}$ unit cell across different directions. The sample with the [110] out-of-plane orientation was inclined by $35^{\circ}$ and rotated around the [110] surface normal to access the chiral [111] direction. The gyrotropic transmission was calculated as the difference between the left and right circular polarizations averaged over frequency region between 600 and $750 \mathrm{~nm}$. The line is a guide for the eye. Courtesy of Silvia Vignolini [?]

\subsubsection{Film thickness effects}

The film thickness effect was investigated by producing samples with thickness variation from $220 \mathrm{~nm}$ to $450 \mathrm{~nm}$. The layer thickness was measured by cleaving the samples and examining the cross-sectioned edge by Scanning Electron Microscope (SEM).

Small fluctuations in the reflectivity response were observed from $300 \mathrm{~nm}$ to $450 \mathrm{~nm}$ as displayed in Figure 4.8. The thinner film, of $220 \mathrm{~nm}$, showed instead a very different response suggesting that a minimum number of unit cell was required to produce sufficient light absorption and self-inductance.

As expected the transmission decayed exponentially with thickness. The decaying rates of the exponential fittings were then calculated for different wavelengths and plotted in Figure 4.9. Interestingly the decay length were longer for wavelengths close to the strongest propagating modes at around $550 \mathrm{~nm}$. 

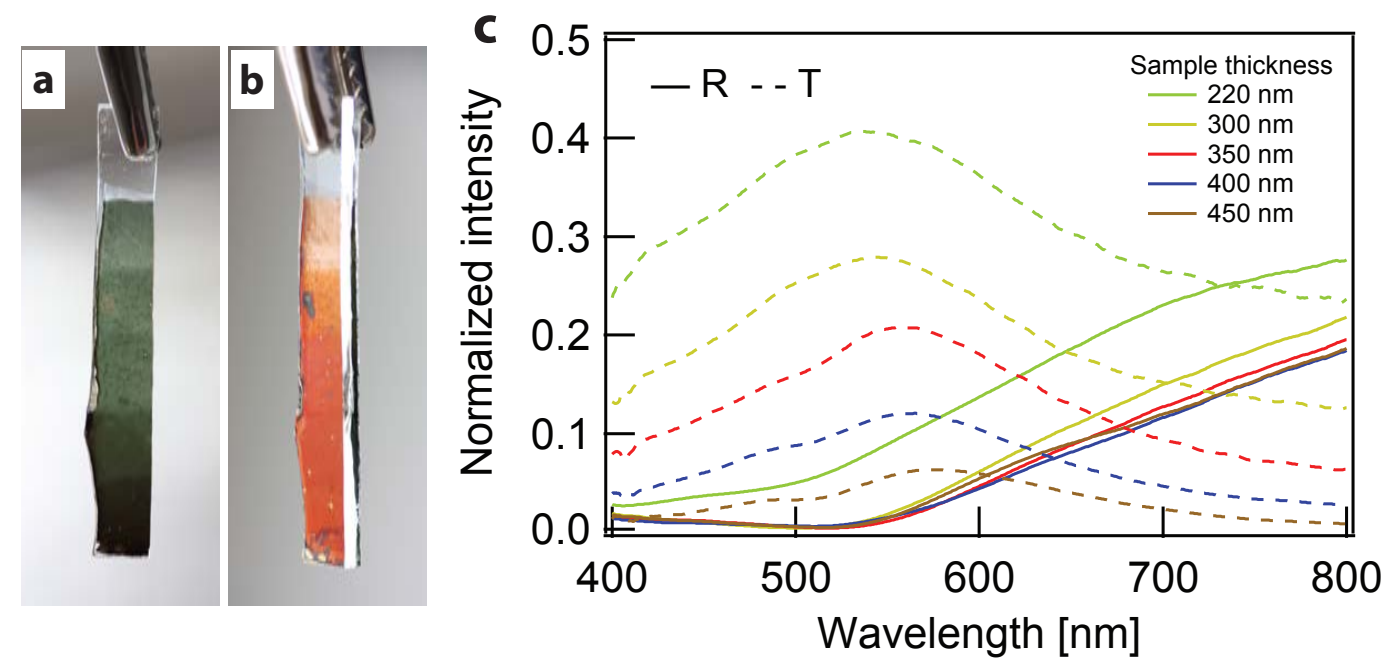

Figure 4.8: (a-b) A gyroid film with increasing thickness photographed in transmission and reflection. (c) Reflection and transmission spectra for increasing thicknesses.

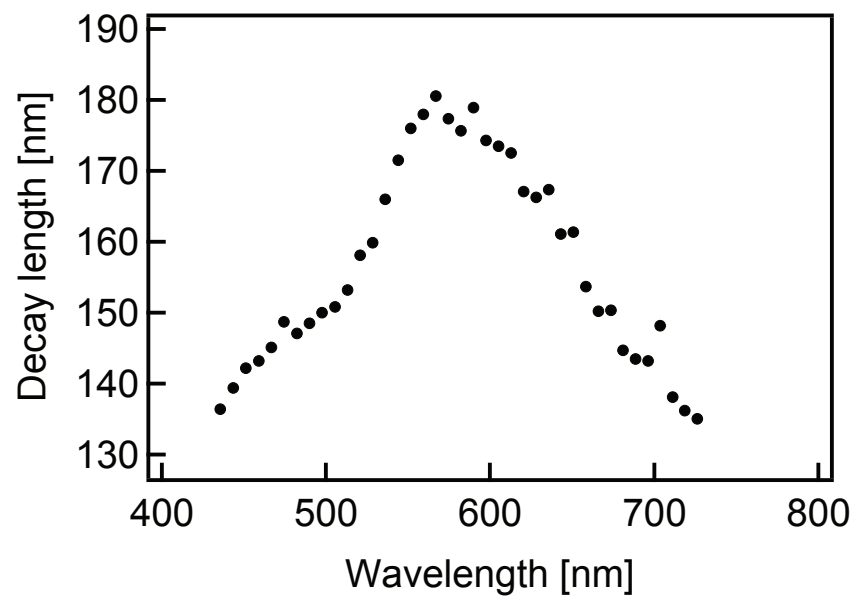

Figure 4.9: Transmission decay length extracted from the exponential decays with thickness for different wavelengths. 


\section{Gyroid metamaterial characterization}

\subsubsection{Disorder effects}

The contribution of the periodicity of the gyroid structure was investigated by inducing a structural distortion via atomic diffusion ? at high temperatures. Atomic diffusion in nanostructures often leads to surface area minimization. The constant mean curvature of the gyroid surface suggests high stability of the gold gyroid against atomic diffusion, but nonetheless, atomic diffusion is favoured to happen towards the grains with more favourable crystal planes on the surface [?].

The result was a distortion of the gyroid structure by the assembly of the gyroid strut into thicker clusters. This process was activated heating the sample at $100{ }^{\circ} \mathrm{C}$ and was followed for 12 hours during which reflection and transmission spectra were taken at constant time intervals (Figure 4.10). While small deformations of the gyroid structure did not significantly affect the effective electron mass and the reflectivity, they did modify the propagation modes. Interestingly, the transmission spectrum was not only reduced in intensity but drastically modified in its shape, suggesting the contribution of different modes of light propagation through the gyroid network.

More drastic distortions were produced at $200{ }^{\circ} \mathrm{C}$ (Figure 4.11). At this temperature the gold gyroid struts were modified into thicker clusters that responded optically very similarly to bulk gold.

Experimental variations The structural deformation activated by atomic diffusion can also explain the small variations of the reflectivity found for different samples. During the removal of the polymer template via plasma etching, the samples were exposed to high temperatures (between 50 and $100{ }^{\circ} \mathrm{C}$ ) for $10 \mathrm{~min}$ utes, inducing small structural distortions.

Experimental variations were also found in transmission. This was strongly affected by the formation of a continuous thin layer between the substrate and the gyroid. Local detachments from the block copolymer film to the FTO substrate were filled with gold during the electrodeposition, producing a continuous thin layer that reduced the transmission (Figure 4.12). The formation of this layer was partially reduced by the substrate functionalization discussed in the previous chapter. 

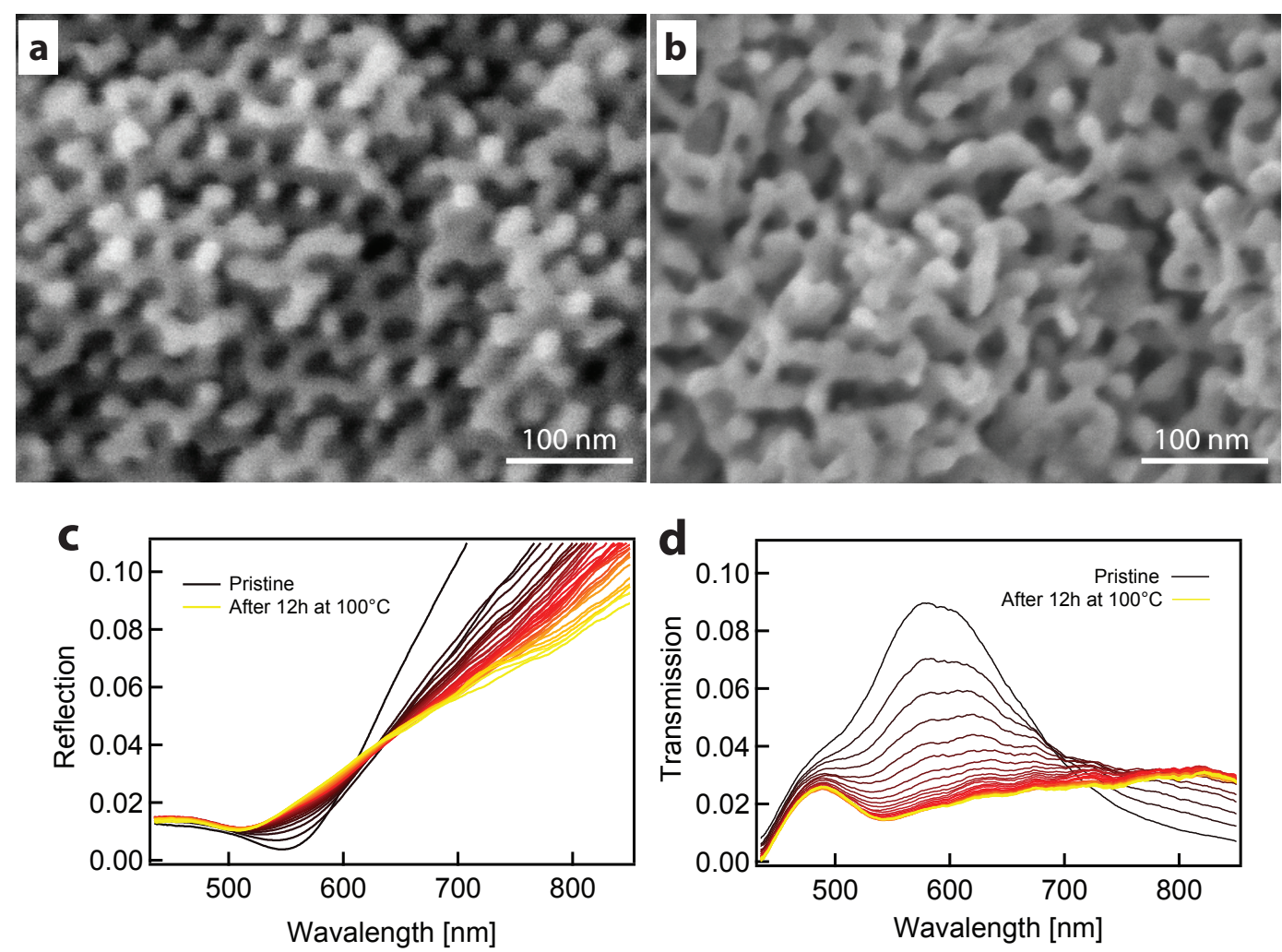

Figure 4.10: (a) SEM image of the gold gyroid before and after heating to $100{ }^{\circ} \mathrm{C}$ for 12 hours. The atomic diffusion activated at high temperature produced a structural distortion that was investigated in reflection (c) and transmission (d). Scan intervals: 20 minutes.

\subsubsection{Double and Silver gyroids}

Due to the lower optical losses, silver is often considered the most promising metal for a metamaterial acting at optical frequencies?. Nonetheless, its practical application in nanostructures is severely limited by the strong tendency to oxidize. Silver gyroid indeed oxidized soon after the removal of the polymer template, producing a change in coloration that varied with the time and across the samples (Figure 4.13).

The oxidation is enhanced by the high surface area and promoted by the high number of structural and electronic defects in nanostructured silver ?. The polymer template could act as an oxidation barrier but the thin unfilled part 


\section{Gyroid metamaterial characterization}
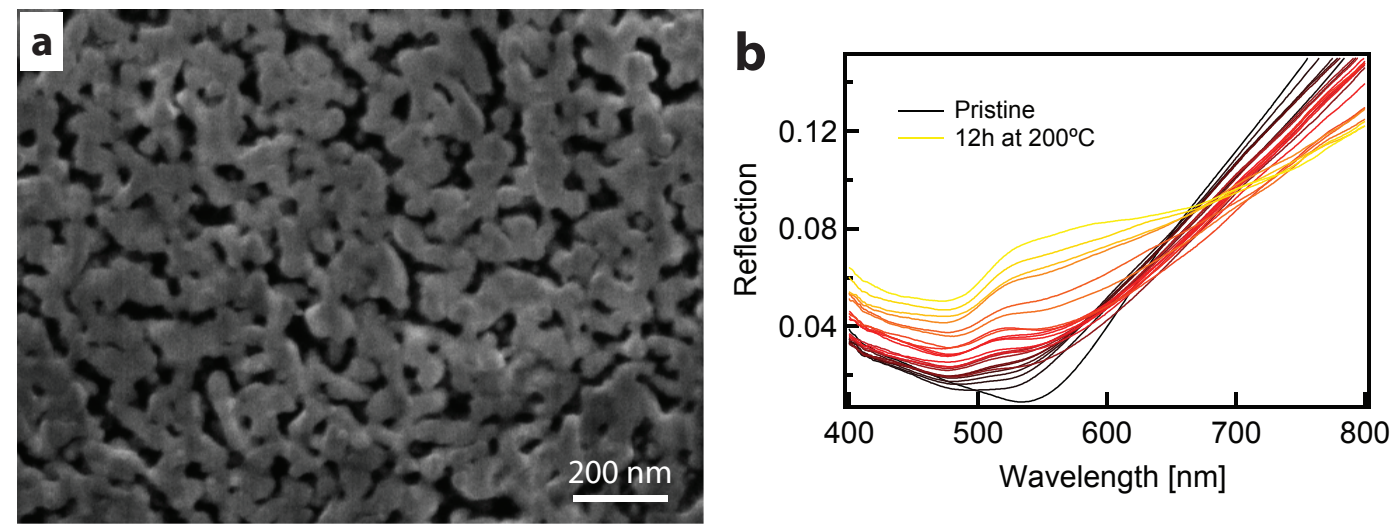

Figure 4.11: a) SEM image of the gold gyroid after heating to $200{ }^{\circ} \mathrm{C}$ for 12 hours. (b) Reflection spectra for scan intervals of 20 minutes.

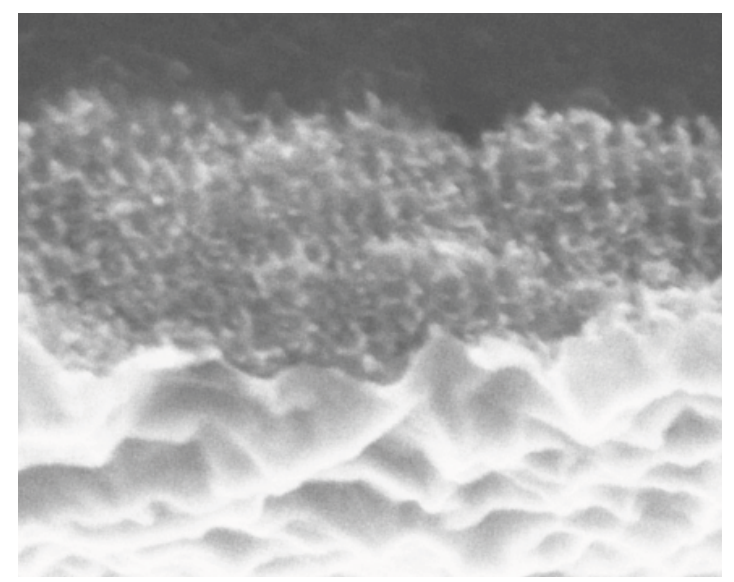

Figure 4.12: SEM image showing a free standing gold gyroid film viewed from the bottom. The continuous thin layer of gold is formed during the electrodeposition at locations where the block copolymer film had detached from the FTO substrate. The morphology of this film resulted from the substrate FTO roughness.

of the template produced strong thin film interference that impeded the optical characterization.

The fabrication method by overgrowth removal, discussed in the previous chapter, enabled the creation of silver gyroid films with flat surfaces that were perfectly filled by the block copolymer template. The polymer, therefore, acted as an oxidation barrier without producing the contribution of additional optical 


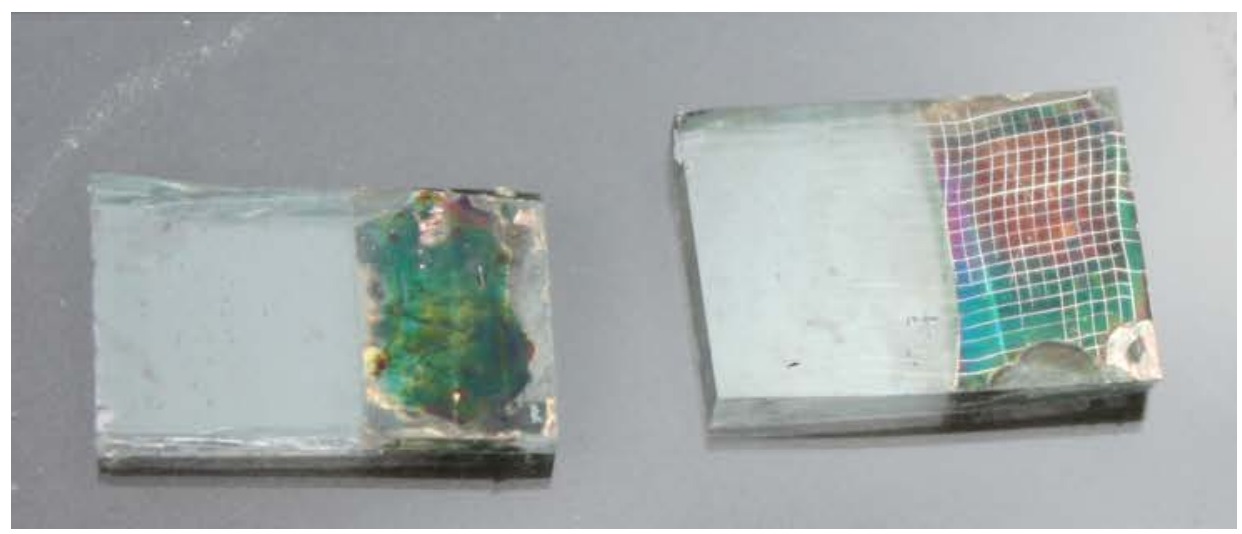

Figure 4.13: Oxidation effects to the silver gyroid after 6 hours (left) and 1 hour (right) after polymer template removal. The color change is a combination of the plasmonic properties of the silver gyroid and the dielectric silver oxide. The right sample was patterned producing a square array.

layers.

Silver and gold gyroids were compared for similar conditions, i.e. with the polymer template still in place and perfectly filled by the metal. As discussed in the next chapter, the presence of the polymer as surrounding medium produces a further reduction of the plasma frequency and a red shift of the reflection spectra. It is reasonable to assume that the effect of the polymer produces similar optical effects in gold and silver gyroids.

Both the reflection and transmission of silver gyroids were characterized by a shift to shorter wavelengths of about $100 \mathrm{~nm}$ with respect to the gold gyroid (Figure 4.14), in agreement with the lower plasma frequency of bulk silver. It is important to stress that these silver and gold gyroid films were produced from similar polymer samples, with a very precise control of the layer thickness.

The effect of the second gyroid network in the double gyroid morphology was also investigated for both gold and silver. As previously described, the double gyroids were produced using diblock copolymer and consisted of a non-chiral structure of two interwoven separate networks.

The second gyroid network was predicted [?] to produce a capacitive effect and act as metal/insulator/metal waveguide, leading to a different light propagation mechanism that stems from coupled surface plasmon resonances of closed loops 

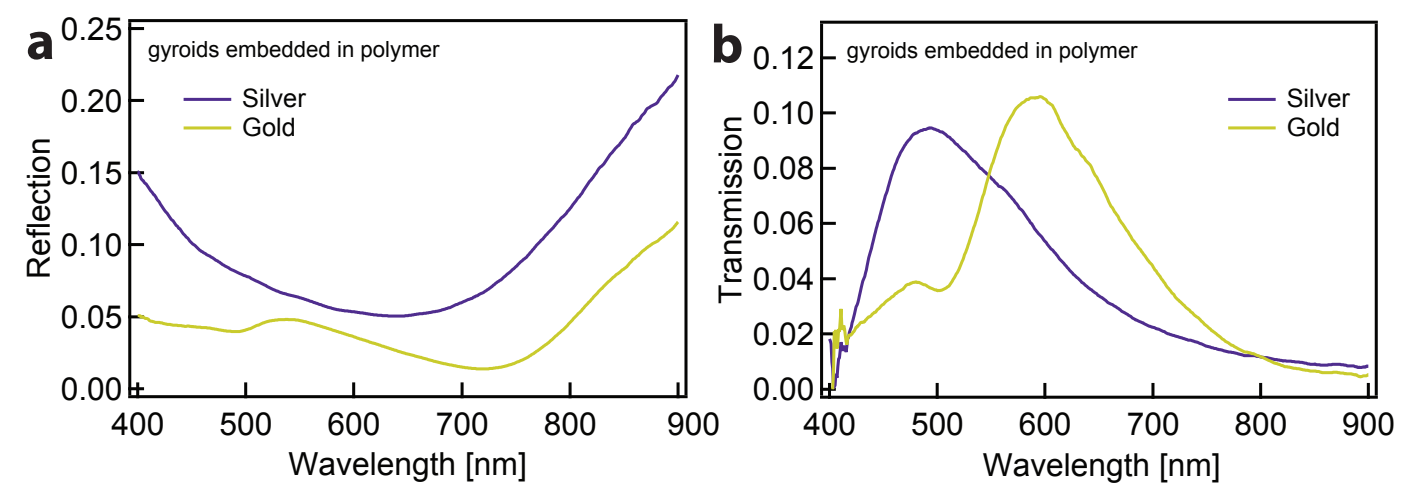

Figure 4.14: (a) Reflection and (b) transmission spectra of gold and silver single gyroids. The samples were characterized with the block copolymer template in place to prevent silver oxidation. As discussed in the next chapter, the surrounding medium with a refractive index of $n=1.6$ (e.gfor polystyrene) produces a red-shift of the reflection spectra. The reflection spectra were for a measured on film sample $1 \mu \mathrm{m}$ thick sample, while the transmission spectra were taken for a $450 \mathrm{~nm}$ thick sample.

of the gyroid interwoven networks.

The optical response of the single versus double gold gyroids were extremely similar, despite of the random orientation of the double gyroid domains and slightly different filling fractions $(f=38 \%$ in the double gyroid and $f=30 \%$ in the single gyroid). However, the difference between single and double gyroids was more evident in the silver gyroids (Figure 4.15).

\subsection{Conclusions}

These gyroid-based structures effectively behaved like metamaterials at visible wavelengths. The two main characteristics were a reduced plasma frequency and enhanced light transmission. The effects of the film thickness and structural ordering were discussed, showing the existence of plasmonic propagating modes in the gyroid structure. This metamaterial exhibited orientation-dependent response under linearly polarized incident light and optical chirality. Silver and double gyroid were also discussed. However, the comparison of single and double 


\section{Gyroid metamaterial characterization}
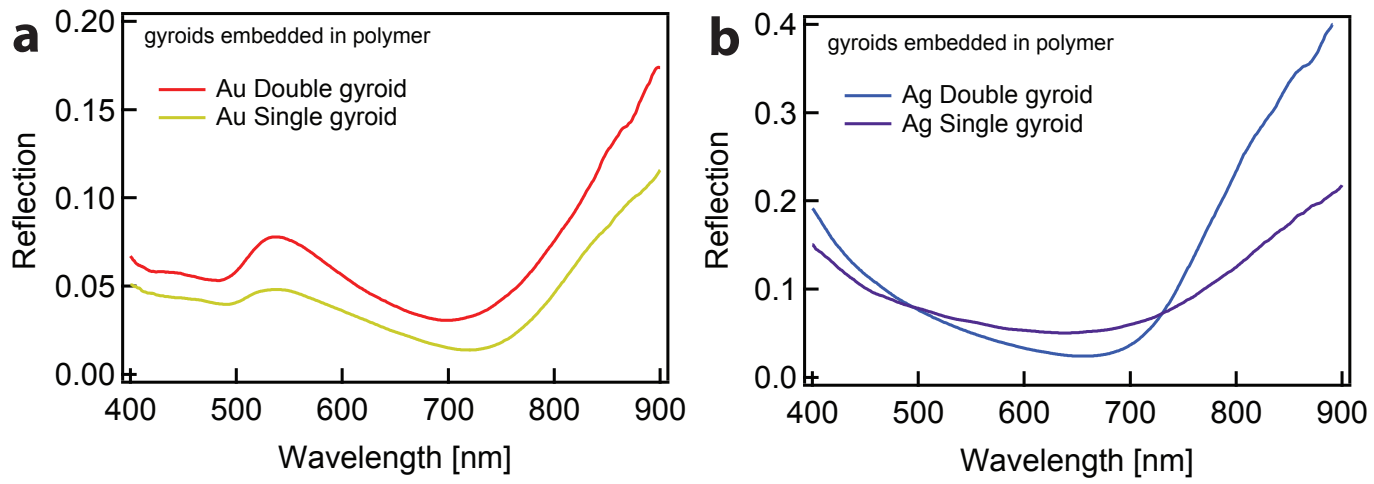

Figure 4.15: (a) Reflection spectra of single gyroid made in gold and silver. (b) Reflection spectra of double gyroid made in gold ans silver.

gyroids was difficult due the different out-of-plane orientation. 


\section{Chapter 5}

\section{Tuning methods}

\subsection{Introduction}

The optical response of the gold gyroid metamaterial was tuned using three different methods as summarized in Figure 5.1. In the first, the structural dimensions of the mesoscopic unit cell were controlled by varying the molecular weight of the structure-forming block copolymer. In the two other strategies using postprocessing, the tuning was achieved by controlling the metal filling fraction of the plasmonic structure and by filling different refractive index media into the metal scaffold?.

The experimental results showed good agreement with finite difference time domain (FDTD)? calculations of the the full 3D structured and also matched an approach based on a three-helix model (THM)?. The FDTD calculations and THM model were provided by Sang Soon Oh and Angela Demetriadou ${ }^{1}$.

The THM described the gyroid architecture more accurately than the wire array model discussed in the previous chapter and assumed that the gyroid's internal structure derives from a network of interconnected metallic helical wires. In particular, there are two types of helices, visible in the [100] direction, with different radii?. The THM showed that the optical properties close to the cutoff wavelength are determined by the smaller helices. This cut-off wavelength originates from the continuous nature of the metallic helices and is related to

\footnotetext{
${ }^{1}$ Ortwin Hess' group, Department of Physics, Imperial College London
} 


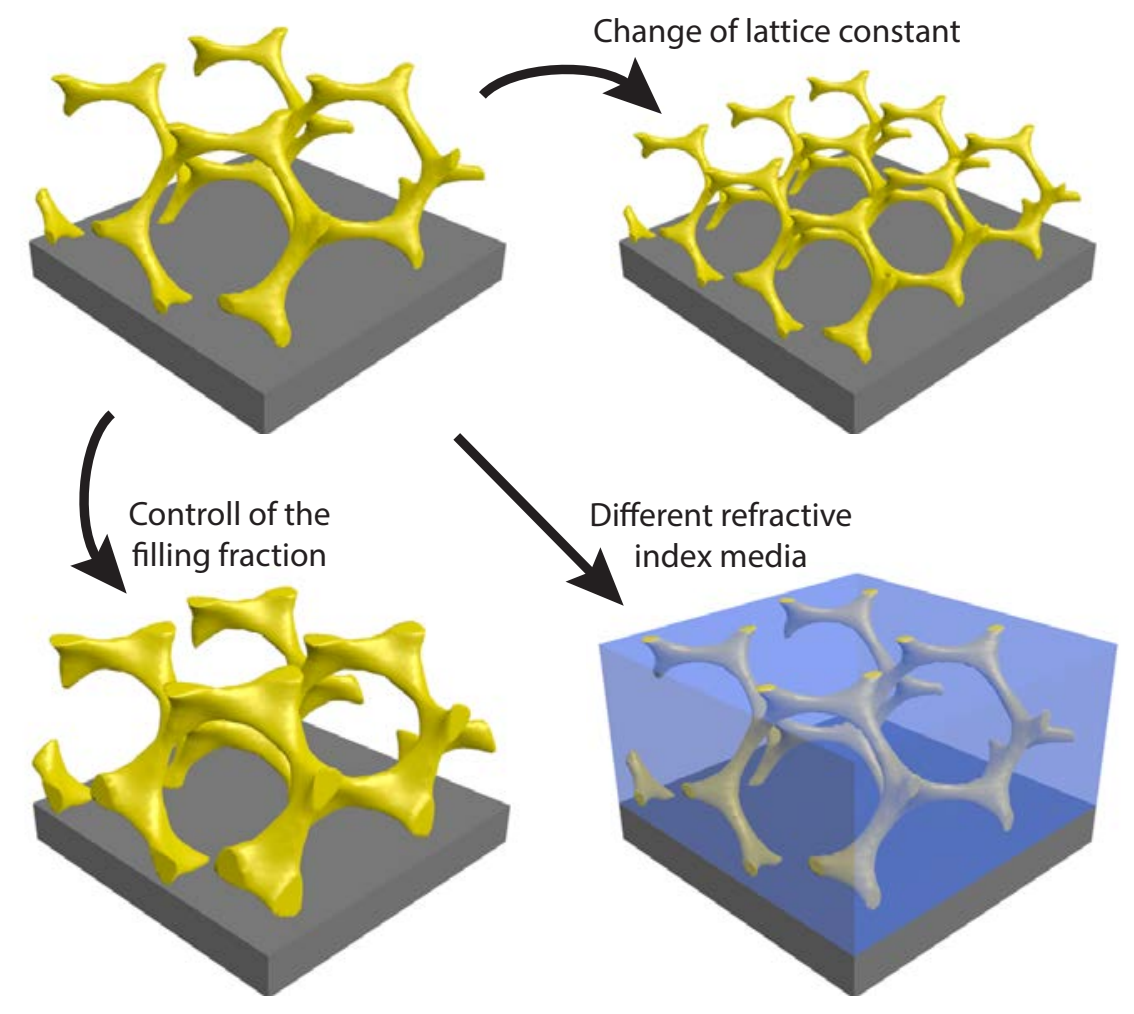

Figure 5.1: Schematic representation of the tuning mechanisms described in this chapter: the optical properties were tuned by structural and environmental variations controlling the lattice constant, the filling fraction and the refractive index of the surrounding medium.

the plasma wavelength. No wave can propagate in the gyroid for wavelengths larger than the plasma wavelength leading to high reflection at these wavelengths. Conversely, three propagating modes were identified just below the plasma wavelength, highly confined within the smaller helices.

As a result the THM model allowed to calculate the plasma wavelength from the following equation which makes use of the geometric parameters of the smaller helices:

$$
\lambda_{\mathrm{p}} \approx c_{1} a\left\{\frac{1}{\epsilon_{\mathrm{Au}} f c_{2}}+\frac{c_{3}}{\epsilon_{n}\left[c_{4} \sqrt{f}-\sqrt{c_{5}+c_{6} \ln \left(c_{7} / \sqrt{f}\right)}\right]^{2}}\right\}^{-\frac{1}{2}},
$$


where $f$ and $a$ are the filling fraction and unit cell of the gyroid, $\epsilon_{n}$ is the dielectric constant of the medium (with refractive index $n$ ) the gyroid is immersed in, $\epsilon_{\mathrm{Au}}$ is the dispersive permittivity of gold, and $c_{1-7}$ are geometrical coefficients?

The optical properties can therefore be tailored by varying the geometrical parameters of the metallic gyroid and the dielectric material it is filled with.

The results presented in this chapter were reviewed and published on Advanced Materials?.

\section{$5.2 \quad$ Unit cell control}

The influence of the lattice constant on the optical properties was studied by comparing two gyroid morphologies obtained from ISO block copolymer with two different molecular weights but identical block volume fractions. The used ISO molecular weights of 33 and $53 \mathrm{~kg} \cdot \mathrm{mol}-1$ correspond to measured unit cell sizes of 35 and $50 \mathrm{~nm}$, respectively, while maintaining the same filling fraction of 30\%. The scanning electron microscopy (SEM) images in Figure 5.2(a-b) show the gold gyroid fabricated from the two ISO triblock copolymers, Figure 5.2(c) compares their reflection and transmission spectra.

As expected, for both lattice sizes the highest reflection values were observed on the long wavelength side of the spectrum and a dip in the spectrum was found when wave propagation in the gyroid slab is permitted (i.e., for $\lambda<\lambda_{\mathrm{p}}$ ). Furthermore, as predicted by the THM model, the $a=50 \mathrm{~nm}$ unit cell has a lower plasma frequency compared to the gyroid with $a=35 \mathrm{~nm}$.

The reflectivity of the smaller gyroid had a minimum at a wavelength of $\sim 550 \mathrm{~nm}$, which shifted to $\sim 620 \mathrm{~nm}$ for the larger gyroid. For larger unit cells and identical filling fractions $f$ (i.e., identical effective electron densities) the gyroid struts were thicker and, as a result, the induced self-inductance was stronger, corresponding to higher effective electron mass per unit cell. Since the effective electron mass is linearly proportional to the plasma wavelength, larger lattice constants led to an increase in the plasma wavelength, as observed in Figure 5.2(c). This property is also clearly evident in Eq.5.1, where for constant $f$, $\lambda_{\mathrm{p}}$ is linearly dependent on $a$. A bigger value of $a$ however significantly increased absorption, resulting in a significantly lower transmission for the larger gyroids. 

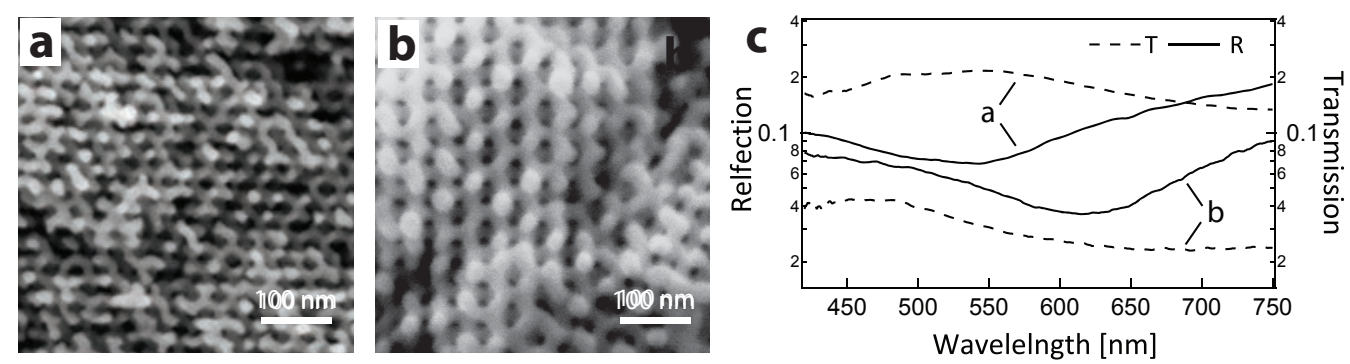

Figure 5.2: (a,b) SEM images of gold gyroid metamaterials obtained from ISO terpolymers of two different molecular weights, yielding lattice constants of 35 and $50 \mathrm{~nm}$, respectively. (c) Corresponding transmission and reflection spectra for unpolarized incident light. Film thickness $\sim 300 \mathrm{~nm}$.

\subsection{Filling fraction variation}

The manufactured gyroids could be post-processed by further gold electrodeposition. In the absence of the surrounding polymer matrix, the entire network structure acted as a cathode and gold growth occurred homogeneously to thicken the gyroid struts without affecting the symmetry of the network. This process continuously increased the filling fraction by thickening the struts, leaving all other parameters unchanged.

Figure 5.3 shows the effect of the filling fraction increase from 30 to $90 \%$, resulting in a reduction in the plasma wavelength and thereby causing a blue shift of the reflectivity edge to shorter wavelengths. As the filling fraction approached $100 \%$ (i.e. solid gold) the reflection spectra indeed approached that of the gold and the reflection minimum disappeared (Figure 5.3(d)).

As the plasma wavelength defined in Eq. 5.1 could not easily be extracted from reflection and transmission spectra, it was convenient to introduce the plasma edge wavelength $\lambda_{\text {pe }}$, which is the wavelength at the point of inflection of the reflectivity (i.e. the wavelength where the first derivative of the spectrum has a maximum, which was easily identified in experimental and calculated spectra, Figure 5.3(e-f)). Although the plasma edge $\lambda_{\text {pe }}$ and plasma wavelength $\lambda_{\mathrm{p}}$ are not identical, they have the same behaviour and differ only by small shift as the gyroid metamaterial is tuned. 

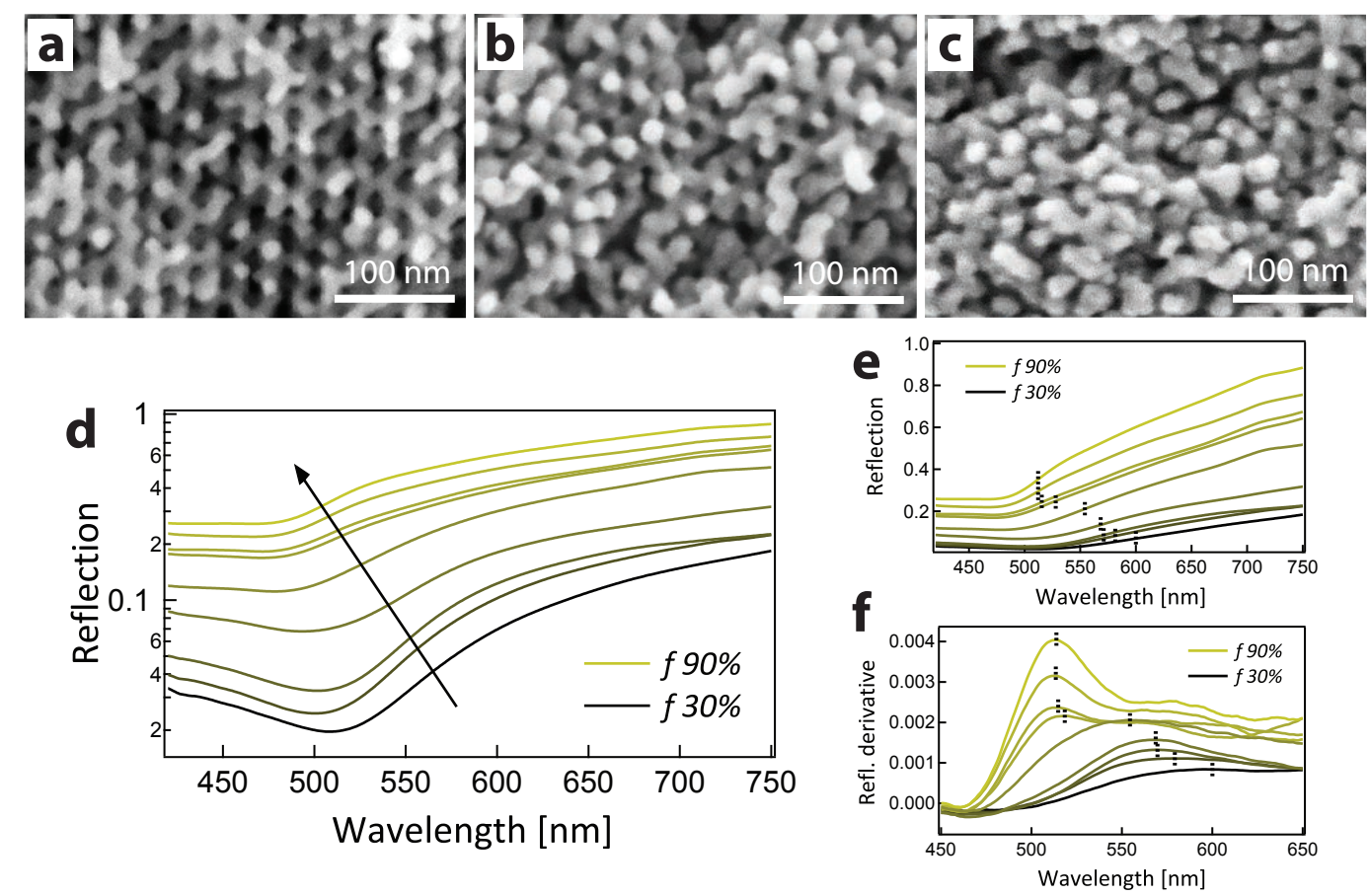

Figure 5.3: (a-c) SEM images of gold gyroids with $a=35 \mathrm{~nm}$ and filling fractions of $30 \%, 60 \%$, and $75 \%$, respectively. (d-e) Reflection spectra for filling fractions varying from $30 \%$ to $90 \%$ in $\sim 8 \%$ increments, in logarithmic and linear scales. (f) First derivative of the reflection spectra highlighting the inflection points (dashed vertical lines). The point of inflection in the reflection spectra was measured calculating the maximum value of the first derivative.

A quantitative analysis of the results in Figure 5.3(d) is shown in Figire 5.4, where the plasma edge wavelength is plotted versus the filling fraction. This analysis reveals a continuous shift in $\lambda_{\text {pe }}$ of more than $100 \mathrm{~nm}$ between solid gold and a gold gyroid with filling fraction of $30 \%$. These results were in excellent agreement with the results of FDTD calculations, as well as with the analytical THM model for the plasma wavelength.

For $f \rightarrow 100 \%$ the plasma wavelength calculated from the THM does not asymptotically lead to the plasma wavelength of bulk gold at $165 \mathrm{~nm}$. Although the THM is unable to predict the behaviour of the gold gyroid at very high filling fractions it is also realistic to expect a drastic change in the plasma frequency from bulk gold to gyroid with $f \rightarrow 100 \%$ due to the continuous porous network 


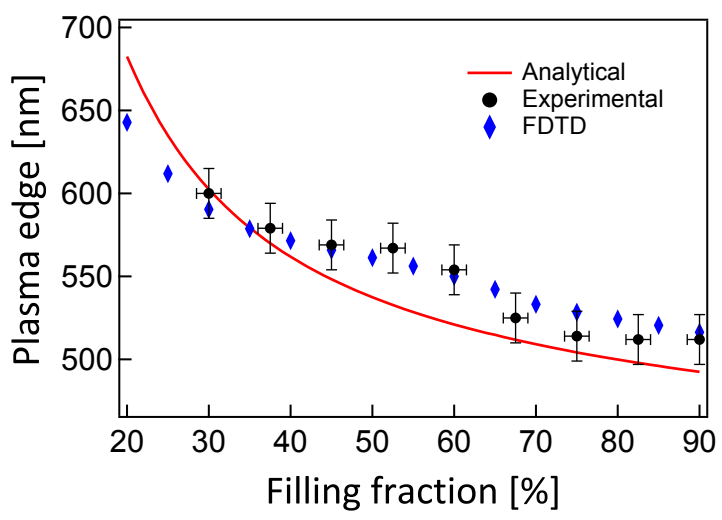

Figure 5.4: (Variation of the plasma edge wavelength $\lambda_{\text {pe }}$ with filling fraction. Experimental data (circles) compared to the THM analytical model (line) and a FDTD calculation (diamonds).

still present at very high filling fractions. Therefore it is sensible to assume the model valid up to $90 \%$ of filling fraction.

Interestingly, despite of the increase of metal content, the filling fraction increment generates a reduction in the optical absorption, as shown in Figure 5.5. While the transmission slowly decays with $f$, the reflection is rapidly enhanced, producing higher absorption for lower filling fraction gyroids than from bulk gold.

The change of filling fraction also affected the linear dichroism in the [110] direction that has been discussed in the previous chapter. Figure 5.6 shows the reflection spectra for different filling fractions and with incident polarizations perpendicular and parallel to the in-plane [100] direction of the gyroid lattice. For $\sim 50 \%$ filling fraction, the linear dichroism is lost. This can be explained considering the origin of the linear dichroism, that arises from a variation of the coupling of polarized light with the differing gyroid lattice symmetries: as the anisotropy of the gyroid was reduced with increasing filling fraction, the dichroism disappeared. 

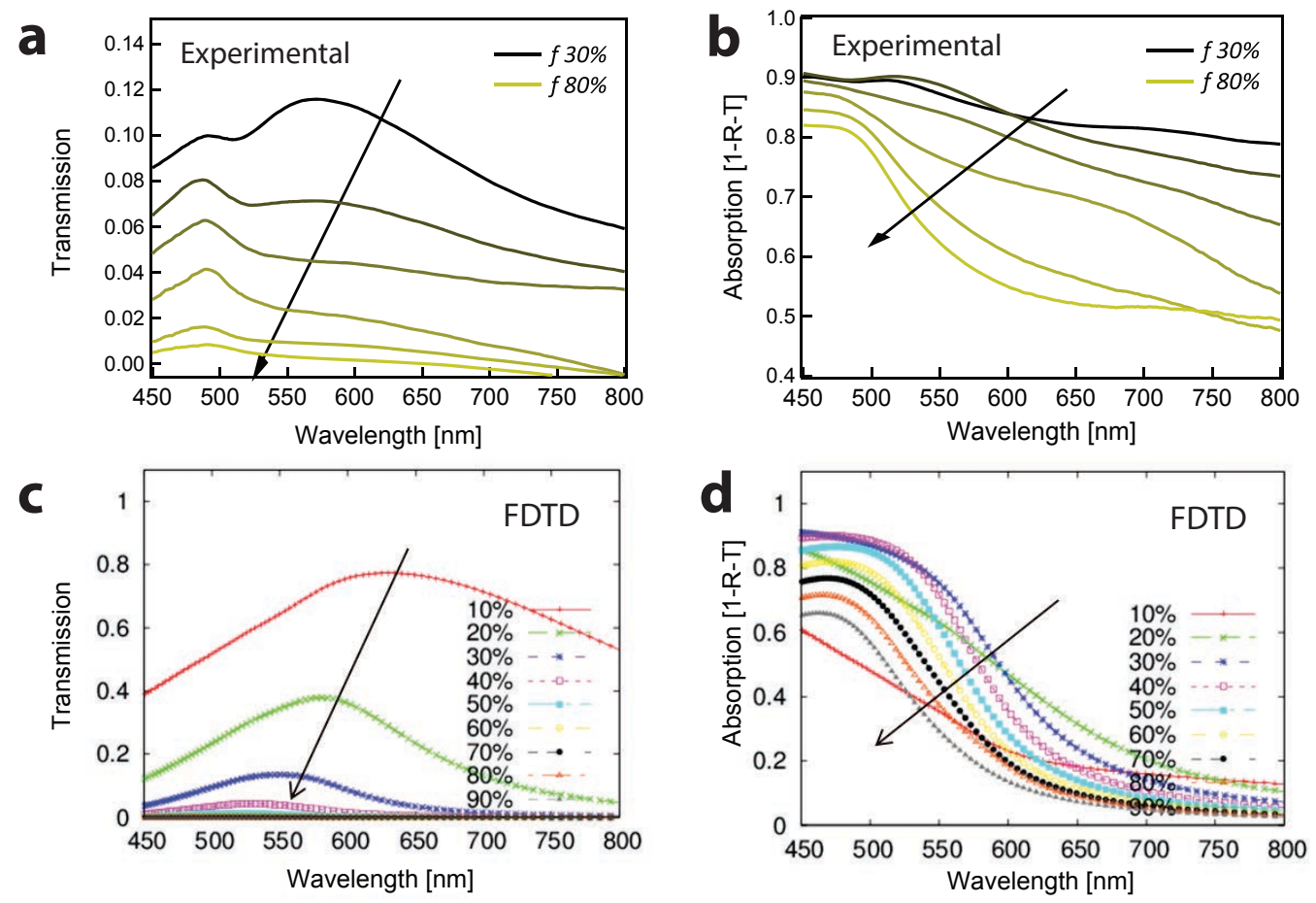

Figure 5.5: (a-d) Transmission and absorption spectra from experimental measurements and FDTD calculations.

\subsection{Surrounding medium effects}

A further way to reversibly tune the optical response of the gyroid metamaterial involved changing the dielectric medium infiltrated into the gyroid. Figure 5.7 shows reflection spectra of the $35 \mathrm{~nm}$ unit cell gyroid with a fill fraction of $30 \%$ immersed in media with increasing refractive index. The plasma wavelength increased linearly with $n$ as illustrated by the variation of the reflectivity curves in Fig. 5.7b. This variation agreed quantitatively with the numerical and analytical results. 

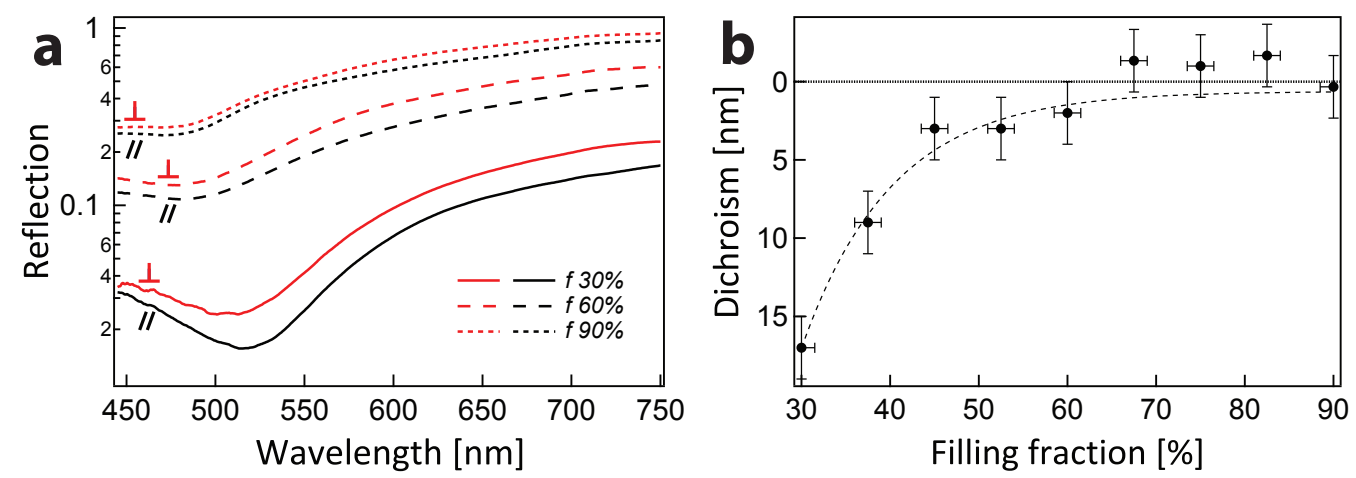

Figure 5.6: Linear dichroism of the gold gyroid in the [110] direction as a function of the filling fraction. (a) Reflection spectra with incident polarization perpendicular (red) and parallel (black) to the in plane [100] direction of the gyroid lattice. (b) Experimental data for the linear dichroism as function of the filling fraction. The dichroism measure was calculated as the difference of the plasma edge wavelengths of perpendicular and parallel polarizations to the [100] direction. The dotted line is a guide to the eye.
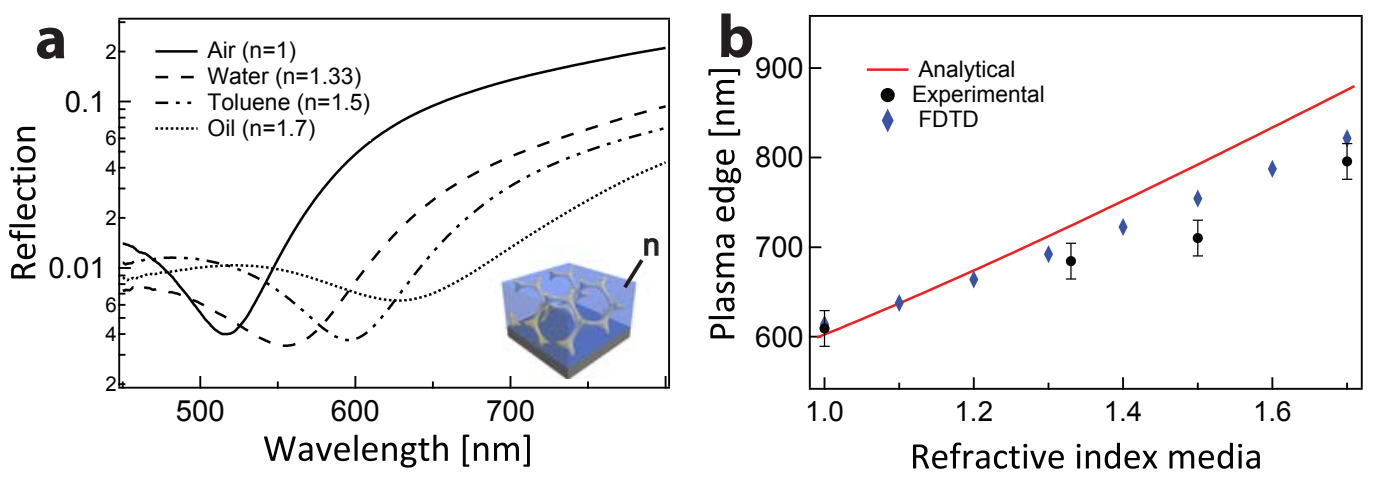

Figure 5.7: (a) Reflection spectra for unpolarized light incident on the $a=35 \mathrm{~nm}$ gyroid filled with dielectric media: air $(n=1)$, water $(n=1.33)$, toluene $(n=$ 1.5), and Cargille Refractive Index Liquid Series B $(n=1.7)$. (b) Variation of the plasma edge wavelength $\lambda_{\text {pe }}$ for different refractive index media compared to the THM analytical model and FDTD calculations. 


\subsection{Conclusions}

In conclusion, three successful approaches were demonstrated to tune the optical behavior of gyroid self-assembled optical metamaterials. These methods can also be applied to other 3D metamaterials.

Optimised structural parameters led to a transmission through $300 \mathrm{~nm}$ thick layers of gyroid-structured gold of up to $30 \%$. Increasing the lattice dimensions in the gyroid metamaterial reduced the plasma frequency and the transmittivity, which was higher for the small unit cell with lower filling fraction. Increasing the gold strut thickness (i.e. the filling fraction) caused a continuous deformation in the shape of the reflectivity curves and increased the plasma frequency towards solid gold. Finally, the optical characteristics could be reversibly tuned by adjusting the refractive index of the medium that is infiltrated into the gyroid pore structure, revealing a linear variation of the plasma edge with $n$. 


\section{Chapter 6}

\section{Hollow gyroid}

\subsection{Introduction}

The gyroid geometry topology discussed above was used as a starting point to create a further complex architecture, the hollow-gyroid with an increased surface area and strongly enhanced optical transmission.

The chapter will commence describing the fabrication technique and the optical properties of the gold hollow gyroid, compared to the gold gyroid discussed in the previous chapters, referred to 'normal gyroid'. I will then discuss the properties of 'composite' metamaterials employing different metals and dielectrics in the gyroidal structure.

A similar fabrication methodology was also pursued to fabricate amorphous carbon hollow gyroids which are relevant in high performance battery applications.

Finally, I will show a similar fabrication methodology to fabricate the inverse gyroid structure mimicking the majority phase of the self-assembled double gyroid block copolymers.

To allow the comparison with the previous results, the characterizations presented in this chapter were all done using $300 \mathrm{~nm}$ thick samples. 


\subsection{Hollow gyroid fabrication}

The ISO triblock copolymer was spin coated onto a FTO-glass substrate, thermally annealed and exposed to UV light to remove the minor polyisoprene phase, as described in Chapter 3. The polymer template was then back-filled with nickel and removed by plasma etching.

The nickel gyroid then acted as working electrode to homogeneously grow gold around the nickel struts. Finally, the inner nickel core was etched away by immersion in iron chloride $\left(\mathrm{FeCl}_{3}\right)$ for 30 minutes, followed by rinsing with deionised water. The fabrication steps, the respective SEM images and the energy dispersive x-ray spectroscopy (EDX) are shown in Figure 6.1.

Interestingly, despite of the gold coating, the nickel core was readily removed by $\mathrm{FeCl}_{3}$. It is reasonable to assume that local defects in the gold coating served as $\mathrm{FeCl}_{3}$ access channels to the Ni-core. Moreover, the thin layer of gold coating was less than $5 \mathrm{~nm}$ thick and consisted of grains with similar dimensions. Interstitial diffusion at the grain boundaries might have also contributed to the etching process.

The electrodeposition of gold onto the nickel gyroid struts was obtained by applying a low steady potential of $(-0.7 \mathrm{~V})$ for times ranging from 10 to 40 seconds, depending on the desired final gold filling fraction.

The gold filling fraction was measured by energy dispersive x-ray spectroscopy (EDX) ? before the nickel core removal. EDX is an analytical technique used for the elemental analysis that allows a quantitative measurement of the elemental composition. As the nickel fill fraction was known from the the initial volume fraction of the polyisoprene phase $(\sim 30 \%)$, the elemental ratio between gold and nickel determines the gold filling fraction. Figure 6.1 shows the 1:1 ratio that was used to compare the optical properties of the hollow gyroid with the normal gyroid. The complete removal of the nickel core was also examined by EDX, as shown in shown in Figure 6.1.

\subsubsection{Optical properties}

Comparison of hollow and normal gyroids Reflection and transmission spectra of a normal gyroid were compared with a hollow gyroid with equal filling 

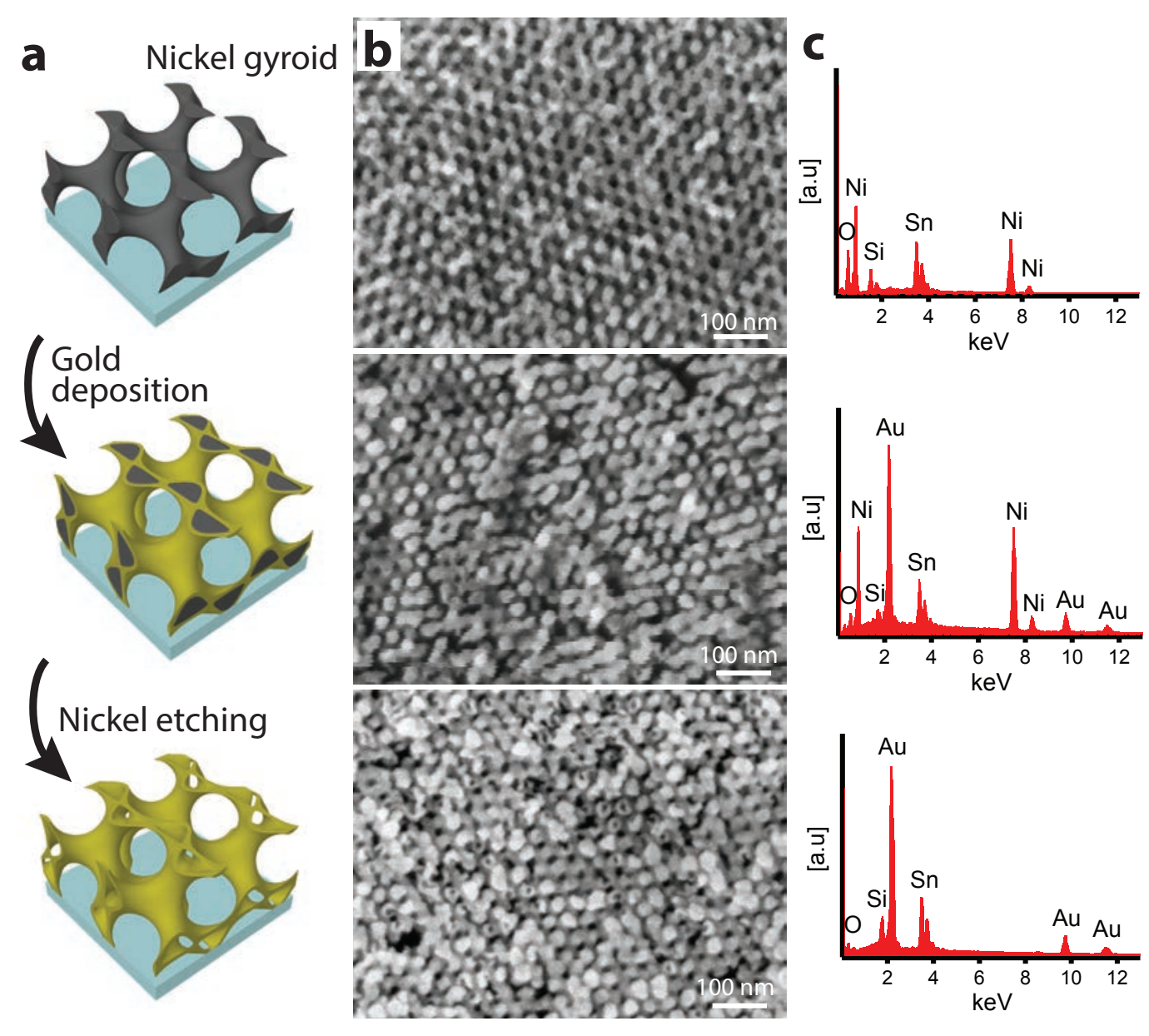

Figure 6.1: (a) Schematic representation of the hollow gyroid fabrication process. A nickel gyroid is coated with a thin layer of gold by electrodeposition and the nickel core is selectively etched away by $\mathrm{FeCl}_{3}$. (b) SEM images of the of the three fabrication steps: after the gold coating the gyroid struts appear thicker and after the nickel etching the hollow cavities are clearly visible along a strut cross-section. (c) Energy dispersive x-ray spectroscopy (EDX) measurements for the three fabrication steps. The ratio between nickel and gold masses at the second steps allows to identify the filling fraction of gold. The complete nickel removal is confirmed by the EDX analysis after $\mathrm{FeCl}_{3}$ etching.

fraction and are shown in Figure 6.2. Despite incorporating the same amount of gold, the reflection and transmission intensities were considerably higher in the hollow gyroid. The reflection edge of the hollow gyroid is shifted to smaller wavelengths with respect to the normal gyroid. This shift is similar to that pro- 
duced by increasing the filling fraction of the normal gyroid, seen in the previous chapter. This behaviour is reasonable considering that in both the hollow gyroid and the high- $f$ normal gyroid have increased outer strut radii.

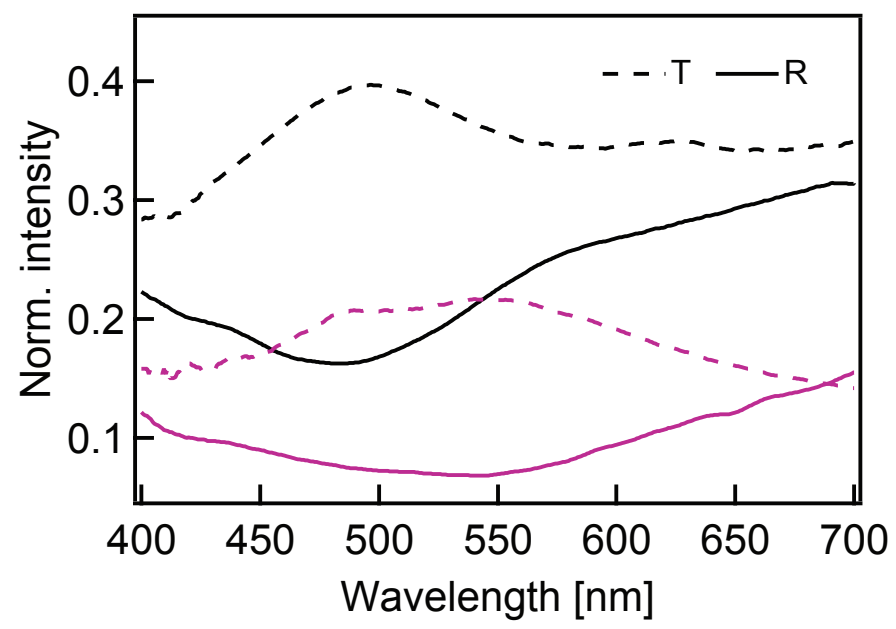

Figure 6.2: Reflection and transmission spectra of the gold hollow gyroid compared with the normal gyroid discussed in the previous chapter. Film thickness $300 \mathrm{~nm}$.

Remarkably, the transmission of the hollow gyroid was enhanced by a factor of 2 with respect to the normal gyroid. As previously discussed, the high transmission through the gold gyroid was caused by the propagating modes through the gyroid architecture. In the hollow gyroid, the surface propagating modes can propagate along both the inner and outer interfaces of the hollow struts, increasing the transmission. Moreover, the pipe-like morphology have thinner walls compared to the strut-width of a normal gyroid with an equal filling fraction. Consequently, the evanescent modes which decay exponentially in the metal, experience lower losses in the hollow structure, as seen in Figure 6.2 where the sum of transmission and reflection is substantially increased compared to the normal gyroid.

Thin hollow gyroid Hollow gyroid with thinner strut wall widths were produced by electrodepositing gold for shorter times. The gold filling fraction was 
again measured by EDX, comparing the amount of gold to the known fraction of nickel. Reducing the gold fill fraction from $30 \%$ to $20 \%$ and $10 \%$ increased the transmission to $\sim 48 \%$ and $\sim 58 \%$, which are remarkable values for $300 \mathrm{~nm}$ thick gold films (Figure 6.3).

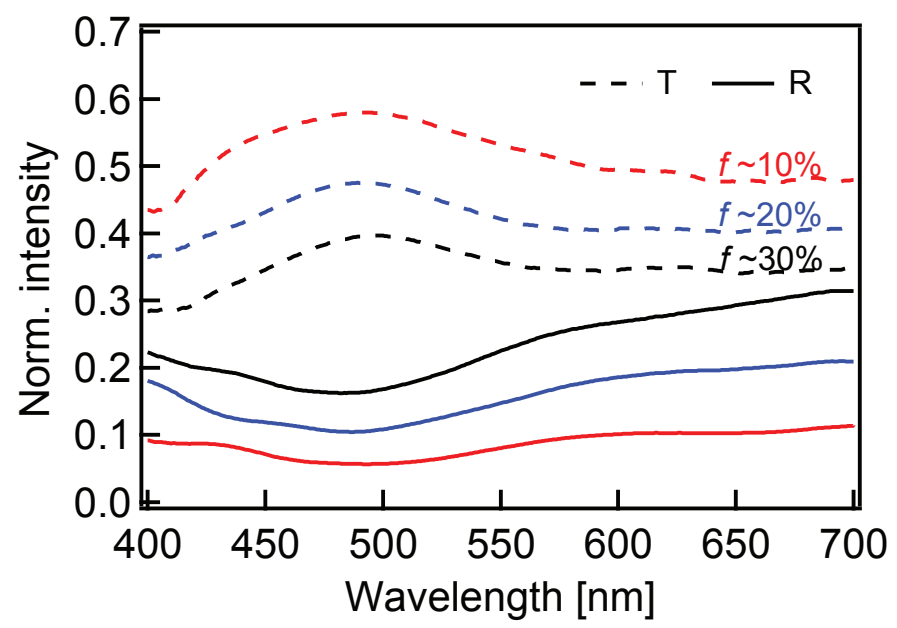

Figure 6.3: Reflection and transmission spectra of gold hollow gyroids for filling fractions of $30 \%, 20 \%$ and $10 \%$. The filling fraction was measured by EDX analysis before nickel core removal.

To compare the transmission of different filling fractions the transmission peaks $T$ can be normalized to the air fraction. The so-normalized transmittance increases from $T_{\text {norm }}=57 \%$ for $f=30 \%$ to $T_{\text {norm }}=60 \%$ and $T_{\text {norm }}=64 \%$ for $f=20 \%$ and $f=10 \%$ respectively. Where $T_{\text {norm }}$ was calculated multiplying the transmission peak by the filling fraction.

Composite hollow gyroid The gold coated nickel gyroid is a metal-composite optical metamaterial. Although the nickel does not have plasmonic properties at visible wavelengths, there has been an increasing interest in nanoantennas made of a ferromagnetic material due to their ability to combine an optical plasmon resonance with strong magnetic properties ??.

Optical antennas are devices designed to convert optical radiation into localized energy, enhancing the interaction between light and matter?. However, the 
plasmonic behaviour in ferromagnetic materials such as nickel is strongly reduced by losses compared to noble metals such as gold. A common strategy to overcome this strong damping is the development of hybrid structures consisting of noble and ferromagnetic materials to increase the plasmon response ??. Therefore, the study of the combined optical properties of composite $\mathrm{Ni}$-Au gyroids is of interest.

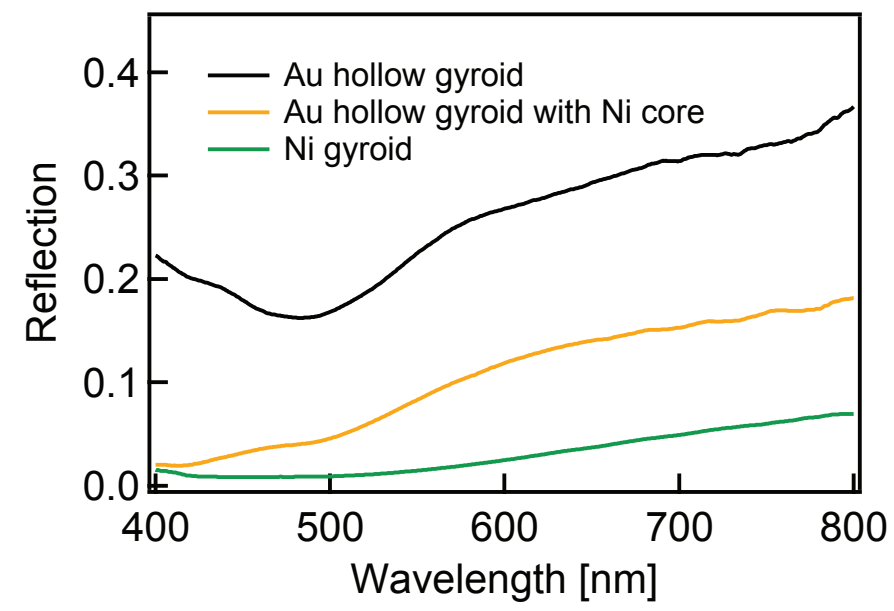

Figure 6.4: Reflection spectra of the gold hollow gyroid compared to the gyroid with a nickel core and to the nickel-only gyroid. The transmittance of the nickelcontaining structures was negligible and are not shown.

As expected, the nickel gyroids had strong optical losses and high light absorbtion in the entire visible spectra as shown in Figure 6.4. Interestingly, the gold gyroid with nickel core displayed an optical response similar to that of the gold hollow gyroid, suggesting the importance of plasmonic modes on the outer gold shell despite of the high losses in the nickel core.

Insulator-metal-insulator metamaterial Hollow gyroids with dielectric cores were fabricated by electrodepositing gold on nickel oxide ( $\mathrm{NiO}$ ) cores. Nickel gyroids were heated at $500{ }^{\circ} \mathrm{C}$ in air for 12 hours to promote complete oxidation. The thin layer of gold was then deposited using a pulsed voltage of $-1.1 \mathrm{~V}$ for 10 cycles of 0.5 seconds. The higher deposition voltage was required because of the low $\mathrm{NiO}$ conductivity. 
The $\mathrm{NiO}$ effectively acted as a dielectric with a refractive index $n=2.18$, forming an asymmetric insulator-metal-insulator metamaterial. When two metaldielectric interfaces are placed close together, the surface plasmons of each interface overlap, giving rise to coupled modes. Cylindrical waveguides with high refractive index core and metal cladding have recently been investigated ?, showing that the coupled modes of asymmetric interfaces (metal-dielectric ${ }_{1}$ and metaldielectric $_{2}$ ) support slow-light propagation, producing degenerate forward and backward waves.

In the hollow gyroid with asymmetric dielectrics, the reflection spectra had two distinctive edges as shown in Figure 6.5. In the previous chapter the effect of an increased refractive index of the medium surrounding the gyroid struts reduced the plasma frequency and produced a red-shift of the reflection spectrum. The two reflection edges in the asymmetric hollow gyroid can therefore be explained as a result of the two interfaces, the metal-NiO interface producing the shift at $\sim 700 \mathrm{~nm}$ and the metal-air interface producing the edge at shorter wavelengths. In Figure 6.5 the hollow gyroid with asymmetric dielectrics is shown together with the reflection spectum of a $\mathrm{NiO}$-only gyroid, showing the characteristic fringes of dielectric thin film interference.

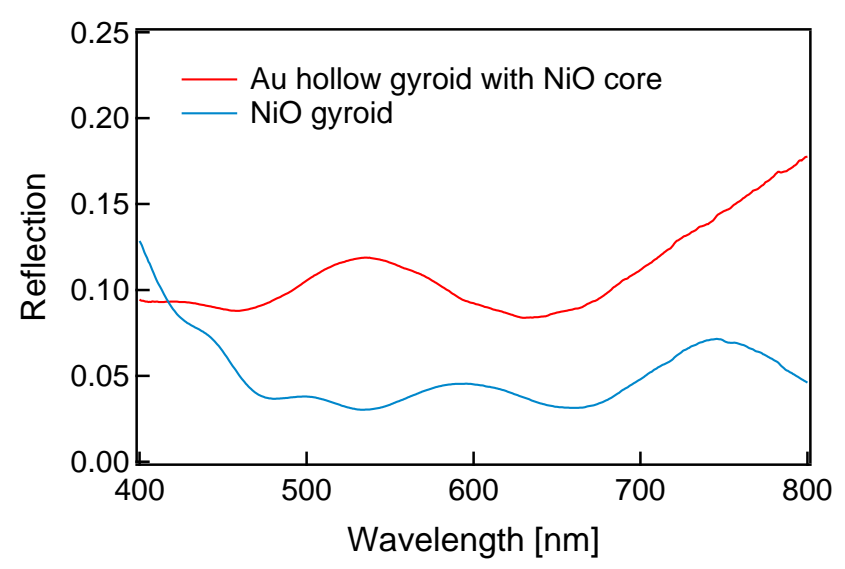

Figure 6.5: Reflection spectra of the gold gyroid with a nickel oxide (NiO) core. The refractive index of $\mathrm{NiO}$ is $n=2.18$ 


\subsection{Carbon hollow gyroid}

Similarly to the fabrication process of the gold hollow gyroid, the nickel gyroid structure was employed as sacrificial frame to fabricate amorphous carbon hollow gyroids. Nickel gyroid samples were heated to $500^{\circ} \mathrm{C}$ for times varying from 5 to 30 minutes in a custom-build cold wall reactor and exposed to $\mathrm{C}_{2} \mathrm{H}_{2}$ to initiate graphitic carbon growth by chemical vapour deposition (CVD). This process was carried out by Piran Kidanmbi ${ }^{1}$ ?. The nickel core was then removed by $\mathrm{FeCl}_{3}$ and the samples were characterised by SEM (Figure 6.6).

The hollow carbon gyroid structure is interesting as high surface are anode in lithium ion batteries. Carbon materials are used in battery systems because of to their unique combination of chemical, electrical, mechanical, and thermal properties ?. The performance of lithium ion batteries, such as the charge/discharge capacity and voltage profile, depend strongly on the microstructure of both electrodes ?. The high surface-to-volume ratio of the carbon hollow gyroid makes this structure ideal to design of carbon/lithium ion batteries with high charge/discharge capacity.
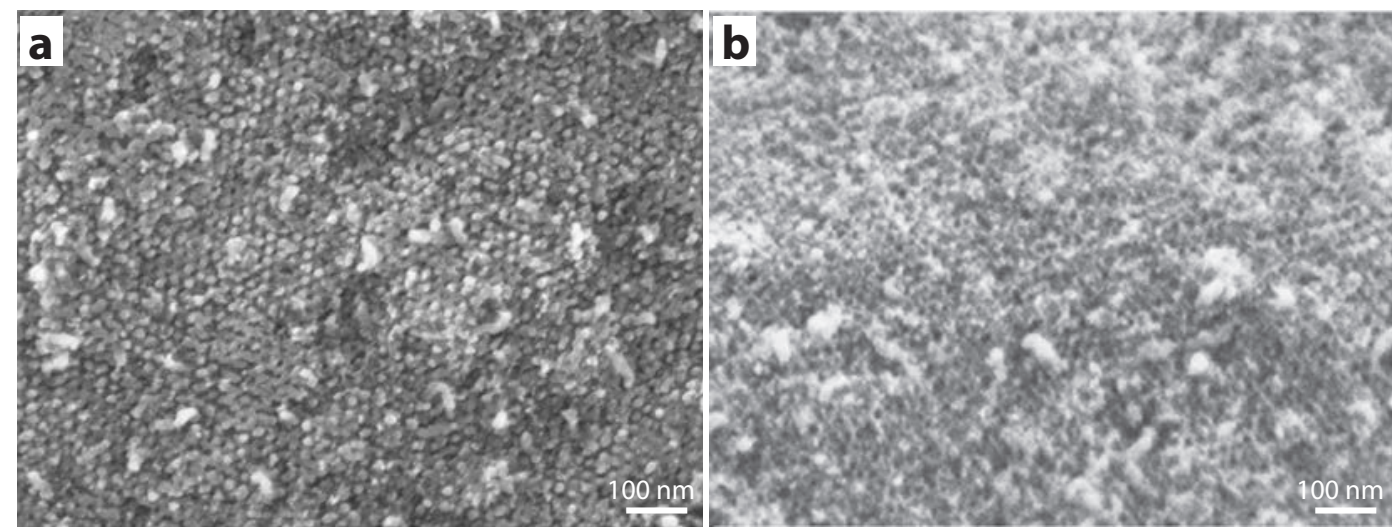

Figure 6.6: (a) SEM image of the nickel gyroid coated with amorphous carbon by CVD. (b) SEM image of the hollow carbon gyroid after nickel etching.

\footnotetext{
${ }^{1}$ The Hofman group, Department of Engineering, University of Cambridge
} 


\subsection{Inverse gyroid}

The fabrication of a structure similar to the hollow gyroid was attempted with the aim to reproduce the majority phase of the gyroid diblock copolymer. Similarly to the hollow gyroid, this geometry divides the space into two separate parts and has two interfaces. Unfortunately the majority phase of the diblock copolymer is composed of polyfluorostyrene and can not be selectively etched, impeding its replication via the standard methodology. Nonetheless, its replication was attempted starting from the nickel double gyroid with a process shown in Figure 6.7.

Gold was slowly electrodeposited onto the nickel strut netwrok until it completely filled the void netwrokk, followed by overgrowth for several microns above the gyroid film. The overgrowth was removed in a similar fashion as described above. The overgrowth layer was removed by sonication and the nickel part was then selectively etched by $\mathrm{FeCl}_{3}$ giving rise to the gold inverse structure of the double gyroid.

It is important to note that the sonication process cleaved the gold at the film-overgrowht boundary. This is presumably a consequence of the small grains and the high number of intergrain dislocations of the gold produced by electrodposition. Such a cleavage did not affect the nickel gyroid, which therefore acted as protective medium for the gold within the gyroid structure. It is also important to remark that several structural imperfections can be expected from this fabrication process, deriving from diffusion problems during electrodeposition preventing the complete filling of the gyroid void structure. The SEM image of the resulting structure is displayed in Figure 6.8 together with the optical characterization.

Despite the presence of two interface in the inverse hollow gyroid, the transmission is significantly lower. This is a consequence of the high filling fraction of this structure of $f \approx 62 \%$, the inverse of the double gyroid with $f=38 \%$. This value is however still higher than the transmission measured for the normal gyroid with $f=60 \%$, shown in Figure 5.5(a) of the previous chapter. 


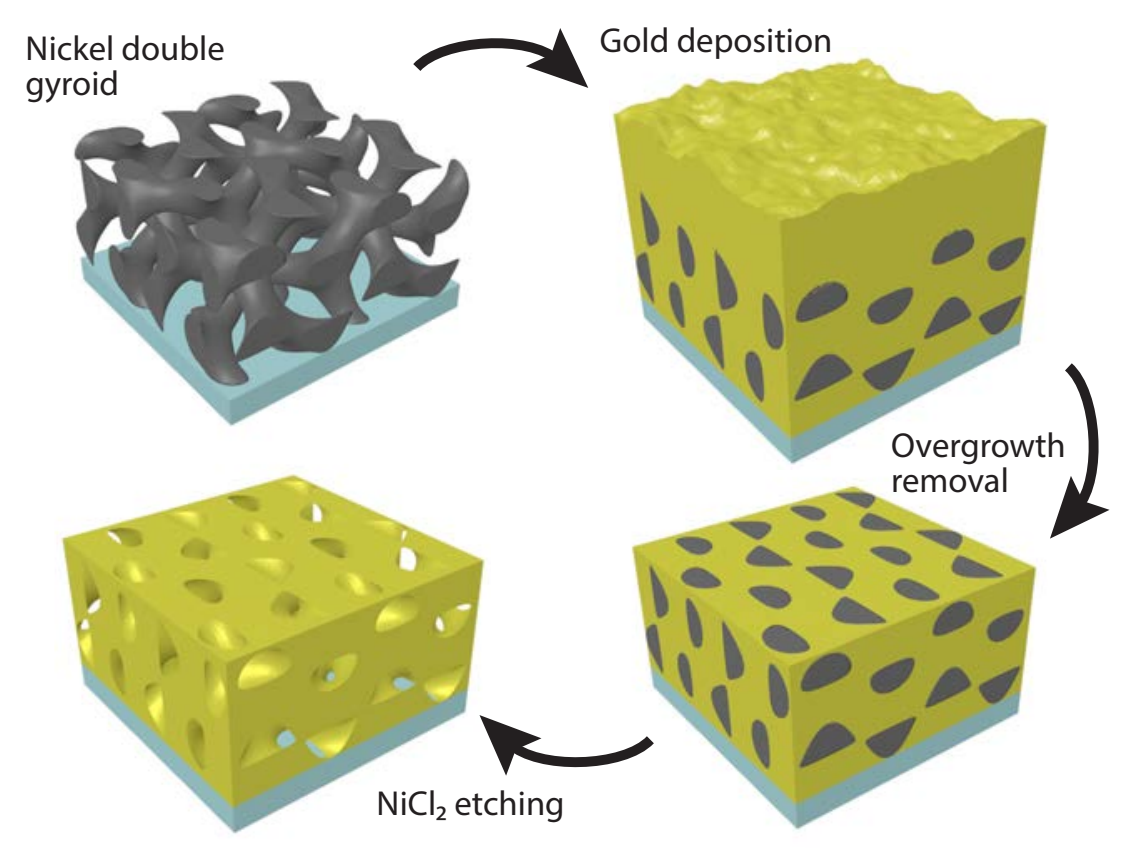

Figure 6.7: Schematic representation of the inverse gyroid fabrication. Gold was electrodeposited onto the nickel double gyroid until complete filling followed by overgrowth of several microns above the film. Subsequently, the top overgrowth layer was removed by sonication. The nickel was then removed by $\mathrm{FeCl}_{3}$ etching.
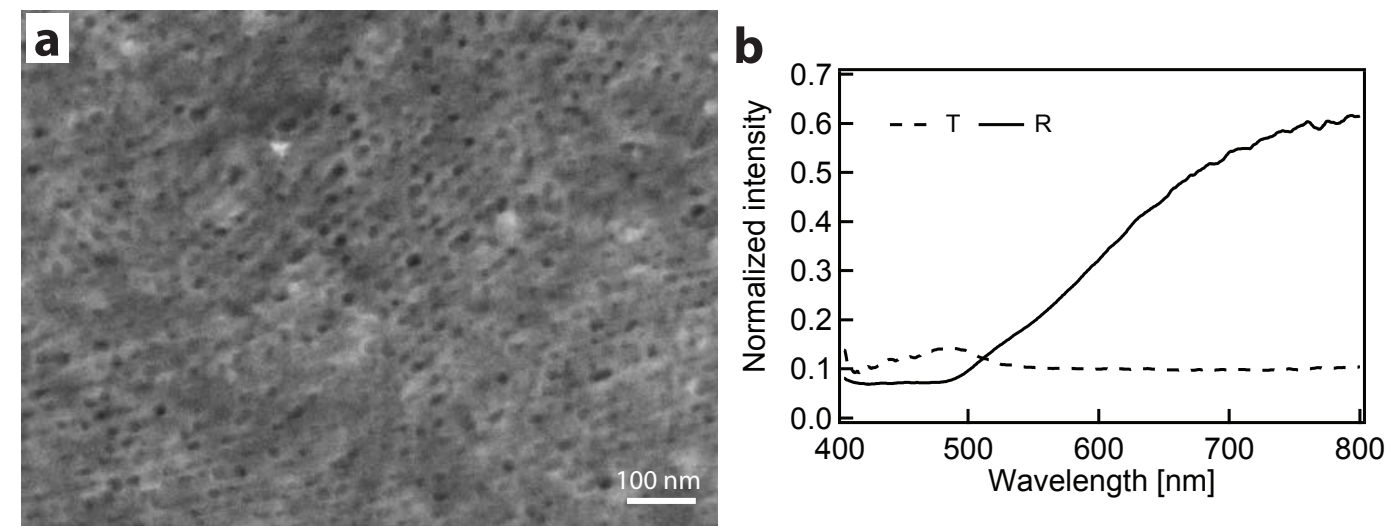

Figure 6.8: Schematic representation of the inverse gyroid fabrication. Gold was electrodeposited onto the nickel double gyroid until complete filling and overgrowth for several microns occurred. Subsequently, the overgrowth was removed by sonication. The nickel was then removed by $\mathrm{FeCl}_{3}$ etching. 


\subsection{Conclusions}

The complexity of the gyroid geometry was further increased by adding an internal interface through the manufacture of a hollow gyroid, producing a significant increase of reflection and transmission.

The hollow gyroid demonstrates that block copolymers can be used not only as a template to replicate their equilibrium phases but also as starting point to create more complex geometries.

Moreover, the combination of different metals in the continuous structure opens new possibilities and gives an additional degree of freedom in the metamaterial design. 


\section{Chapter 7}

\section{Flexible and stretchable gyroid metamaterials}

\subsection{Introduction}

For versatile and practical applications, metamaterials are required to be robust and readily integrated to other systems, adapting their conformation to various geometries. For this reason stretchable and flexible metamaterials can be advantageous in many applications. Flexible metamaterials have been demonstrated at terahertz ? and visible ? frequencies with bidimensional structures.

However, state-of-art tridimensional optical metamaterials are still confined on rigid flat substrates and their nanostructure is easily exposed to structural damage.

In this chapter I will present the fabrication of stretchable and flexible gyroid metamaterials. The change of optical properties upon axial and plane strain deformations will also be discussed.

\subsection{Stretchable metamaterial}

Stretchable metamaterials were fabricated by infiltrating an elastomer into the gold gyroid network and etching the sacrificial layer on which they were grown as depicted in Figure 7.1. The glass substrate was initially coated with a $10 \mu \mathrm{m}$ thick 
layer of nickel and functionalized with silane as described in Chapter 3 for ITO substrates. Gyroid block copolymers were then produced by thermal annealing and replicated into gold using the standard procedure.

A PS- $b$-PI- $b$-PS elastomer was dissolved in anisole $\left(10 \mathrm{wt} \%, M_{\mathrm{w}}=8 \mathrm{~kg} \cdot \mathrm{mol}^{-1}\right)$ and infiltrated into the gyroid network by drop casting. The solvent was slowly evaporated at room temperature, leaving the gyroid metamaterial embedded in a thick transparent rubber matrix.

The infiltration process was strongly affected by the molecular weight of the elastomer. Long polymer chains, such as commercial polydimethylsiloxane (PDMS) could not diffuse into the gyroid network due to the fact that the pore size of the gyroid network was smaller than the elastomer chain lengths.

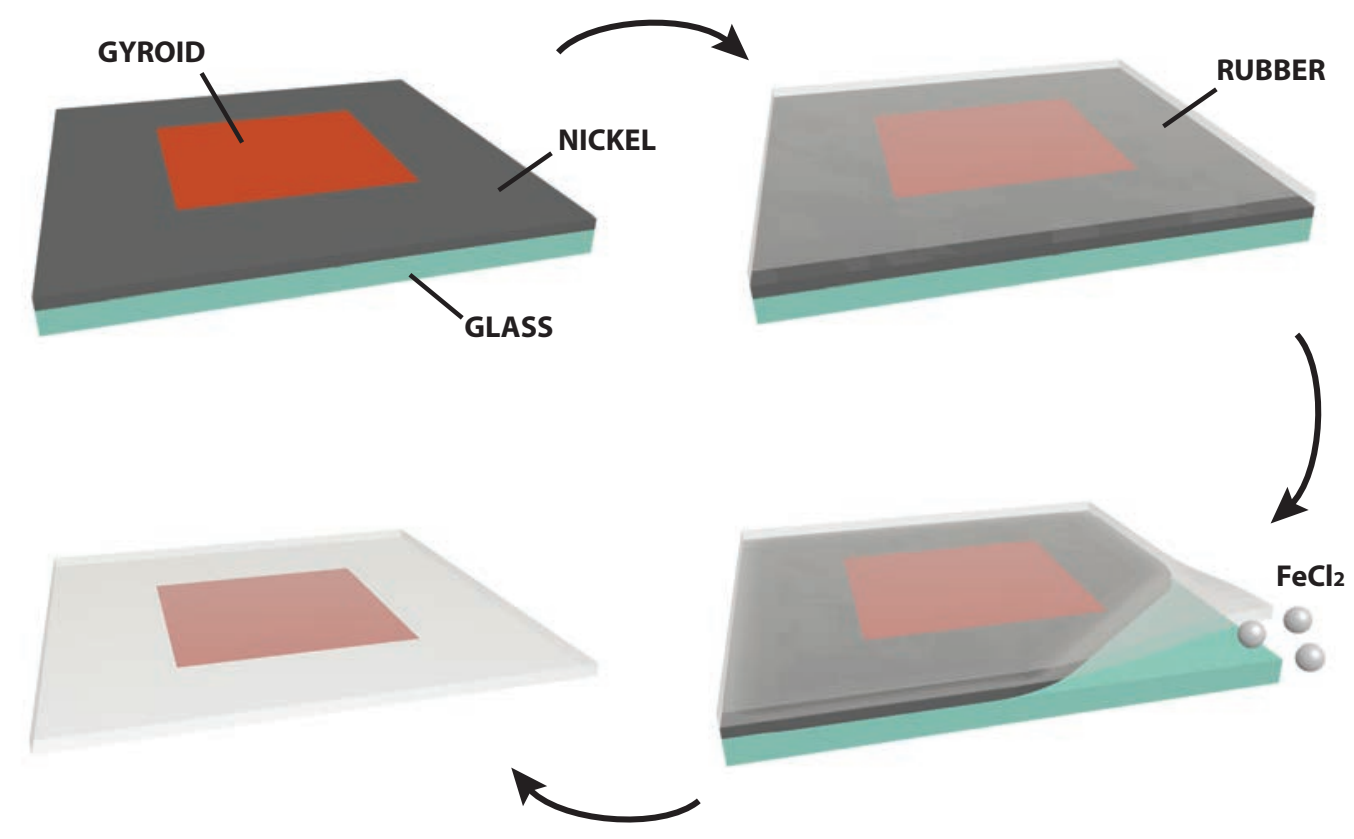

Figure 7.1: Schematic of stretchable metamaterial fabrication. A gold gyroid was fabricated on a nickel-glass substrate and an elastomer was infiltrated into the gyroid network by drop casting. Finally, the nickel layer was etched way by iron chloride, leaving the gyroid metamaterial embedded in a transparent rubber matrix.

The etching of the nickel layer allowed to obtain an optical metamaterial that was fully supported and embedded in an elastic and transparent matrix. 

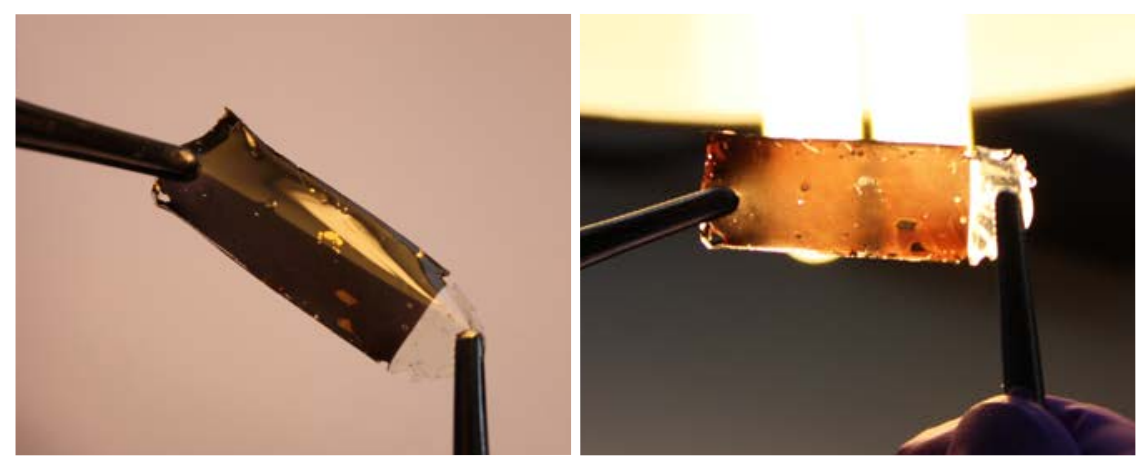

Figure 7.2: Photos of the stretchable metamaterial in reflection and transmission. The width of the sample is approximately $4 \mathrm{~cm}$.

To investigate the optical response of the elongated gyroid, the rubber film was extended using a mechanical stretcher, enabling the strain in one axial direction (and the compression in the orthogonal directions). As shown in Figure 7.3, increasing stretching caused the reflection decrease at long wavelengths and the larger transmission band was slightly reduced.
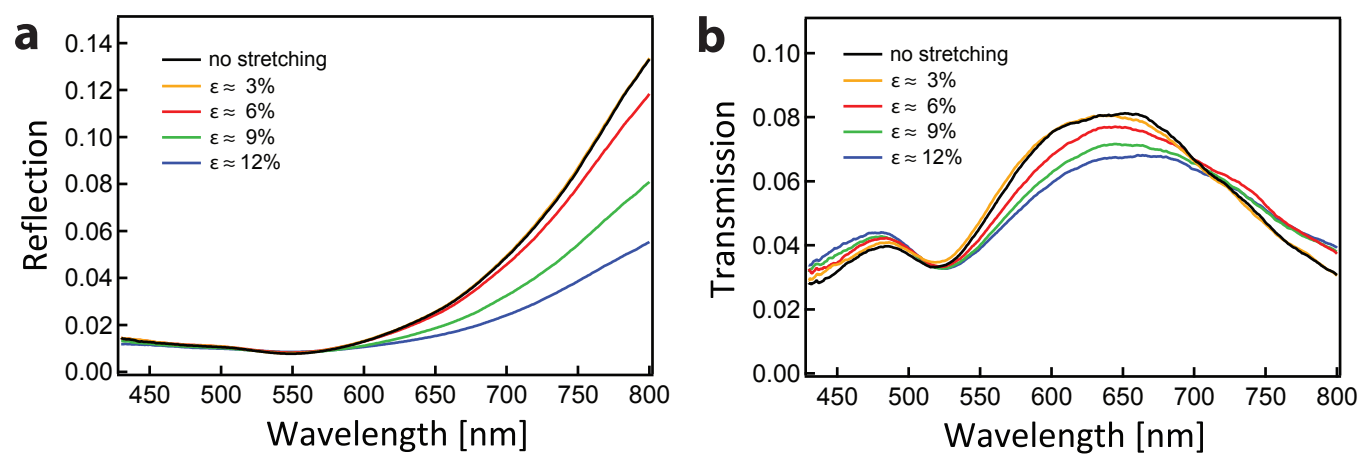

Figure 7.3: Reflection and transmission spectra for unpolarized incident light on stretchable gyroid metamaterial for increasing strains.

The gyroid film embedded in the rubber matrix, however, exhibited some discontinuities and fractures as a result of residual stresses in the elastomer. These were clearly visible in the transmission images shown in Figure 7.4(a). The dimensions of these discontinuous blocks (measured as distance from opposite edges) were monitored during film stretching and were effectively elongated in 
the strain direction (Figure 7.4(b-e)) and compressed in the orthogonal direction, confirming the deformation of the gyroid structure (Figure 7.4).

Large elongations induced additional fractures in the gyroid film, as shown in Figure 7.4(f) that limited the study of the optical response at large deformations.

However, in this analysis, the optical measurements were averaged over different small gyroid domains. These have random in plane orientations, as discussed in the Chapter 3, and were therefore deformed along different axis during the film stretching. The study of the optical response of elongated single domains was conducted on gyroid films fabricated by drying-annealing on flexible substrates.
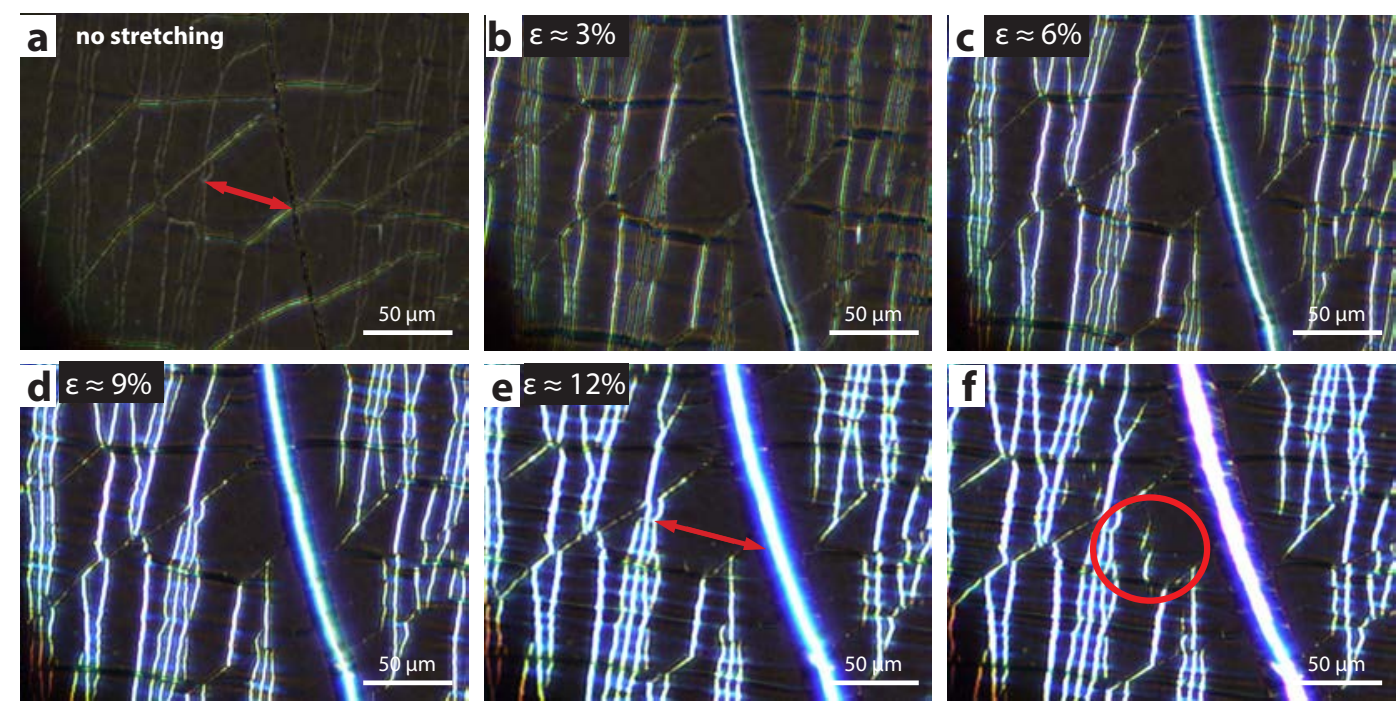

Figure 7.4: Tranmsission images of a gyroid structure under axial stretching. (a) Initial state with no stretching, some discontinuities in the gyroid film were produced by residual rubber stress. (b-e) Stretching of the gyroid film and consequent elongation of the discontinuous blocks. (f) Cracks formation at a high strain.

\subsection{Flexible metamaterial}

Gold gyroids were fabricated by drying annealing on a $500 \mu \mathrm{m}$ thick ethylene tetrafluoroethylene (ETFE) foil coated with nickel. 
The gyroid metamaterial was elongated or compressed by bending the ETFE substrate as shown in Figure 7.5. The strain could be extracted by the radius of curvature using the relation (see Appendix)

$$
\epsilon=\frac{\Delta x}{x}=y / \rho,
$$

where $\rho$ represents the radius of curvature, $x$ is the neutral axis, and $y$ is the distance from the neutral axis. In the elastic range, the tensile and compressive stress-strain curves of ETFE are identical and the neutral axis can therefore be approximated to be at one-half foil thickness.
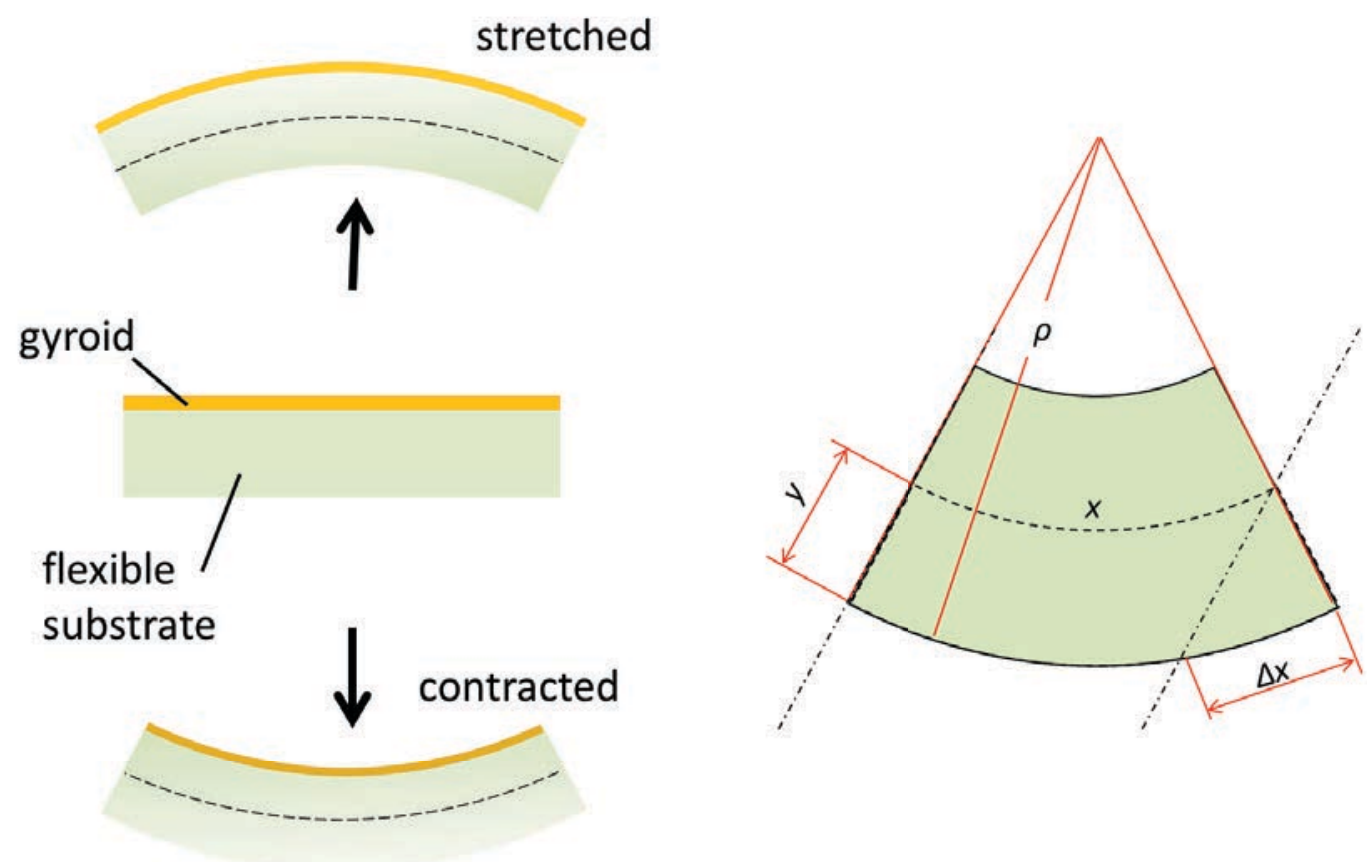

Figure 7.5: Schematic of gyroid stretching by substrate bending.

The reflection spectra were measured on single gyroid domains for unpolarized and linearly polarized incident light. The domains were chosen with the in plane [110] orientation parallel and orthogonal to the elongation axis. As shown in Figure 7.7, only minor variations were observed in the optical response for deformations of $\epsilon=9 \%$. The linear dichroism did not present significant variations in any of the elongated gyroid structures. 
For deformations larger than $\epsilon=9 \%$, the gyroid film was fractured in the direction orthogonal to the strain axis. I note that these fractures occurred for smaller elongations than the gyroid stretched in the rubber matrix system. The reason may arise from the different strain states. In the rubber system the gyroid was elongated by axial strain (Figure 7.6(a)), with consequent compression in the $z$ and $y$ directions, while on the curved substrate, the gyroid was elongated by plane strain (Figure 7.6(b)), with no variations in the $y$ direction and a larger compression in the $z$ direction. In structural failure analysis the latter represents a more critical strain state due to the higher compression in the orthogonal direction $?$.
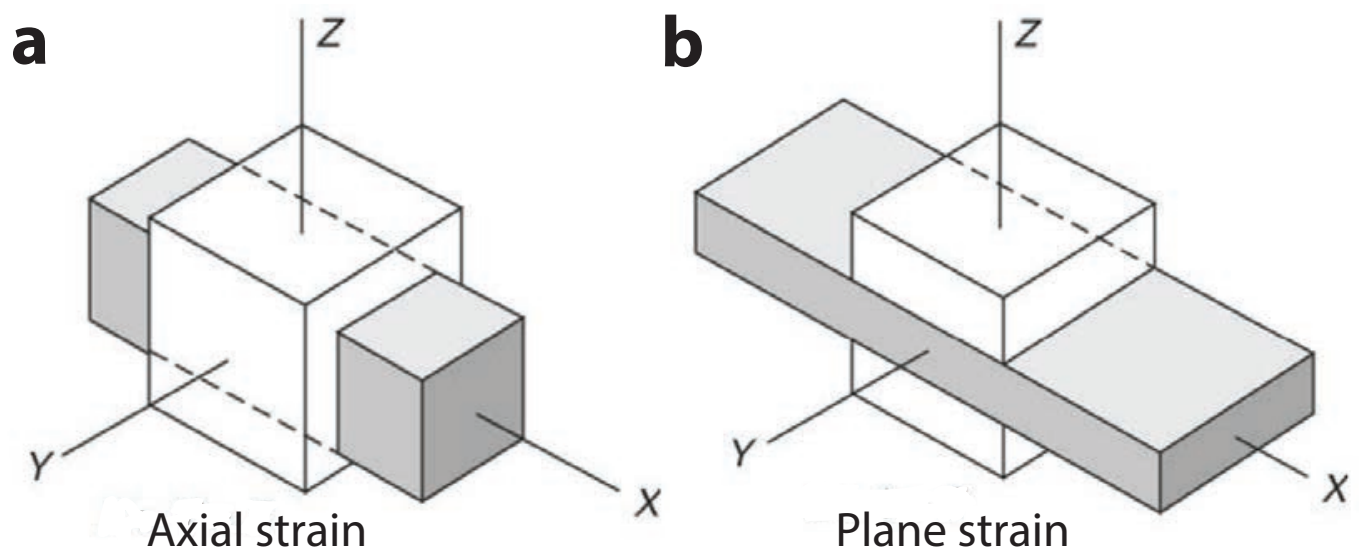

Figure 7.6: Schematic of gyroid elongations. (a) Axial strain is induced in the rubber system stretching; (b) plane strain is a result of substrate bending.

Interestingly, larger optical variations were found in compressed gyroid as shown in Figure 7.8. Reflection of polarized and unpolarized incident light resulted in a decrease of intensity with compression. However, the linear dichroism did again not show a significant change with deformation.

Simulation spectra from FDTD calculations were computed by Sang Soon $\mathrm{Oh}^{1}$ for axial deformations and are presented in Figure 7.9. These calculations suggested a significant variation of the optical response only for large deformations, which were experimentally unattainable because of fracture. However, the

\footnotetext{
${ }^{1}$ Ortwin Hess' group, Department of Physics, Imperial College London
} 

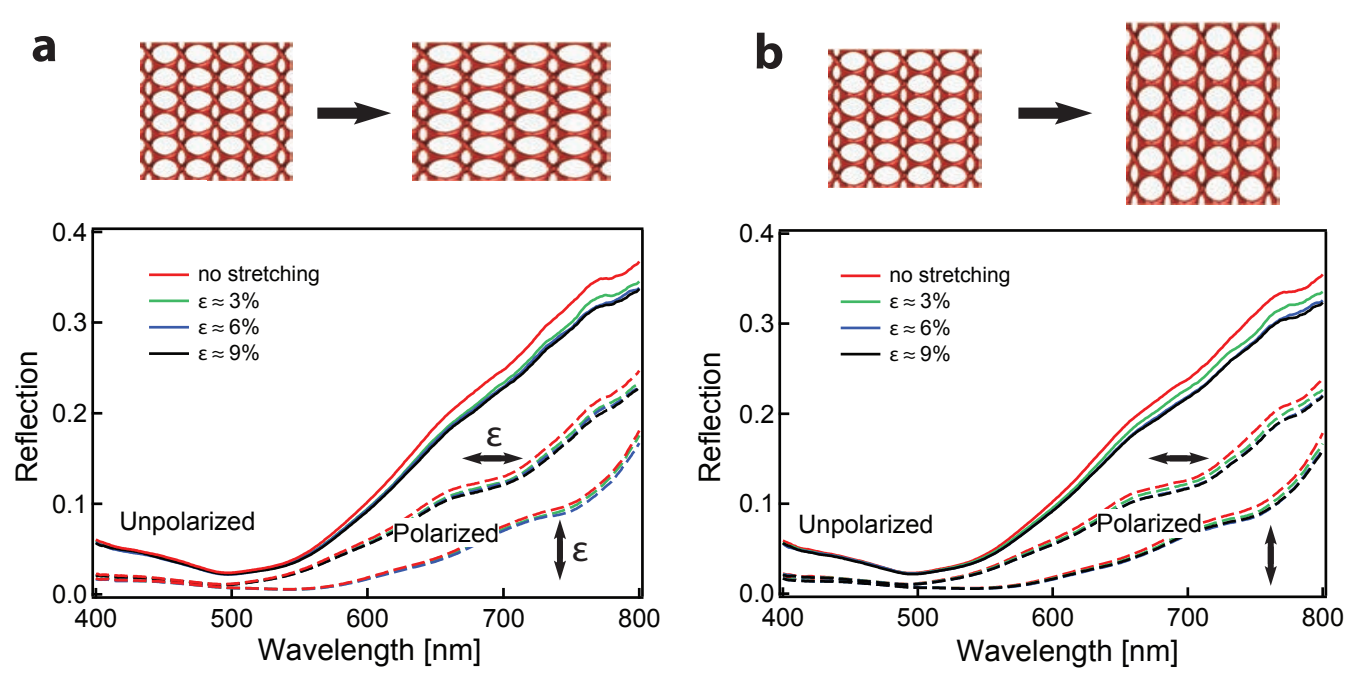

Figure 7.7: Reflection spectra under plane strain for unpolarized and linearly polarized incident light. (a) Gyroid elongated along the in-plane [110] orientation. (b) Gyroid elongated along the in-plane [100] direction.

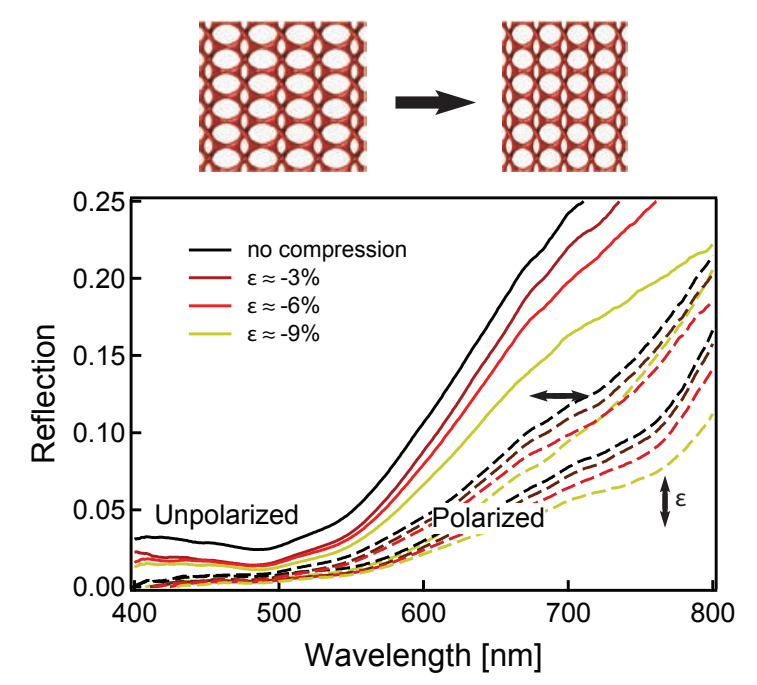

Figure 7.8: Reflection spectra under plane strain for unpolarized and linearly polarized incident light. Gyroid compressed along the in-plane [110] orientation.

optical variations were predicted to be larger in the compressed gyroid, as shown experimentally. 

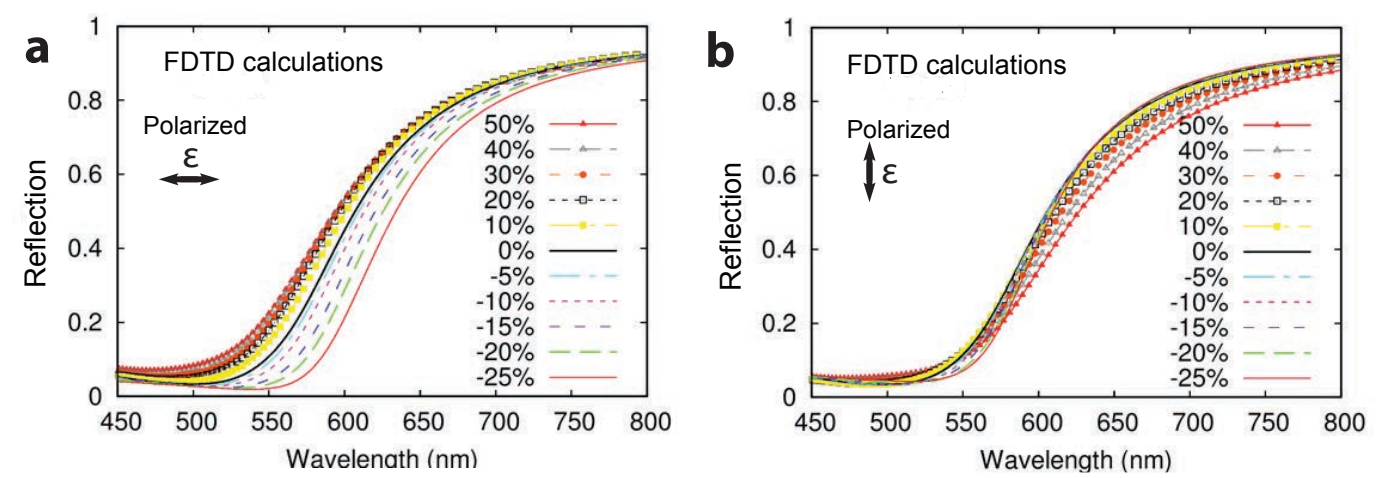

Figure 7.9: FDTD calculations of a stretched and compressed gyroid structure under plane strain along the in-plane [110] orientation. Courtesy of Sang Soon Oh.

\subsection{Conclusions}

The fabrication of stretchable and flexible metamaterials was demonstrated. This represents an important achievement for practical applications. This fabrication method can be easily implemented in other three-dimensional metamaterials.

However, the strain was limited to few percents before inducing structural fractures. The morphology of the elongated gyroid structure will also need to be studied by structural analysis (e.g. x-ray scattering) to investigate how the gyroid unit cell is modified under strain. 


\section{Chapter 8}

\section{Metamaterial sensors}

\subsection{Introduction}

Gas sensing is an important process in a wide range of applications. In petrochemical industries sensors are used to ensure safety and monitor products ?. Other applications include atmospheric science and breath diagnostics ??. The quantitative detections of gases is dominated by laboratory analytical equipment such as gas chromatographs, but these measurements preclude real time data acquisition. Semiconductor sensors can also be highly sensitive, but, they are specific to individual gases and have a limited lifetime.

Metamaterial have been proposed to improve the sensor performances by localized field enhancement ??. For example, resonant modes of a 2D subwavelength resonator have been used for biosensing ??, and split ring resonator have been applied to monitor materials that respond resonantly at $\mathrm{THz}$ frequencies as explosives and DNA ??.

In this chapter I present the application of optical metamaterials as vapour sensors. This analysis offers a fast optical response at specific vapour concentrations and can be made in-situ without disturbing the sample environment.

It represents the first application of metamaterials for vapour sensing with immediate response at optical wavelengths, exploiting the high sensitivity of the metamaterial optical properties to the surrounding environment. This sensing system may fill an important gap between low cost sensors with inferior perfor- 
mance and highly sensitive laboratory equipment.

\subsection{Capillary condensation}

The gyroid metamaterial sensor is based on the capillary condensation of liquid vapours into gyroid structures and on the resulting variation of their optical response. In capillary condensation, the vapour phase is adsorbed into a porous medium to the point at which the pore space are filled with condensed liquid from the vapour. The condensation occurs well below the saturation vapour pressure of the liquid. This is due to an increased number of van der Waals interactions between vapour phase molecules inside the confined space of the capillary ?.

Capillary condensation is an important factor in porous structures. It can be used to determine pore size distribution and surface area ? and can be described by the Kelvin equation:

$$
\ln \frac{P_{\mathrm{v}}}{P_{\mathrm{sat}}}=-\frac{2 H \gamma V_{1}}{R T}
$$

where $P_{\mathrm{v}}$ is the equilibrium vapour pressure, $P_{\text {sat }}$ the saturation vapour pressure, $H$ the mean pore curvature, $\gamma$ the liquid/vapour surface tension, $V_{1}$ the liquid molar volume, $R$ the ideal gas constant, and $T$ the temperature. Different pore geometries result in different types of curvature. The equations for the mean pore curvature $H$ are available at numerous sources ?. For a cylindrical capillary $H=1 / 2 r$, with $r$ the radius of the cylinder.

The key aspect of gyroid structures in capillary condensation is the high monodispersity of their nanometric pores. The capillary condensation can therefore be induced at very specific vapour pressures (i.e. vapour concentrations). Moreover, the gyroid unit cells and pore sizes can be easily varied with the block copolymer molecular weight.

The liquid condensation in gyroid metamaterials produces a shift in reflection towards longer wavelengths. As discussed in Chapter 5, this shift has a linear dependance to the liquid refractive index. This optical change enables the detection of vapour concentrations above a specific limit, set by the gyroid unit cell size. 


\subsection{Solvent vapour sensing}

Gold gyroids with 35 and $50 \mathrm{~nm}$ unit cell sizes were first tested as sensors for water vapour.

The gold gyroids were loaded into a small chamber with a transparent window that allowed the optical characterization of the gyroid exposed to a solvent vapour (Figure 8.1). The concentration of the solvent vapour was controlled by using two streams of nitrogen gas, one of which was passed through a washbottle filled with water, inducing the saturation of this stream before the two streams were mixed and introduced into the gyroid chamber. The flow rates of both streams were controlled by two electronic mass flow controllers, so that the precise adjustment of the relative flow rates controlled the relative solvent vapour pressure $P_{\mathrm{v}} / P_{\text {sat }}$ from 0 to 1 , corresponding to water vapour saturation from 0 to $100 \%$.

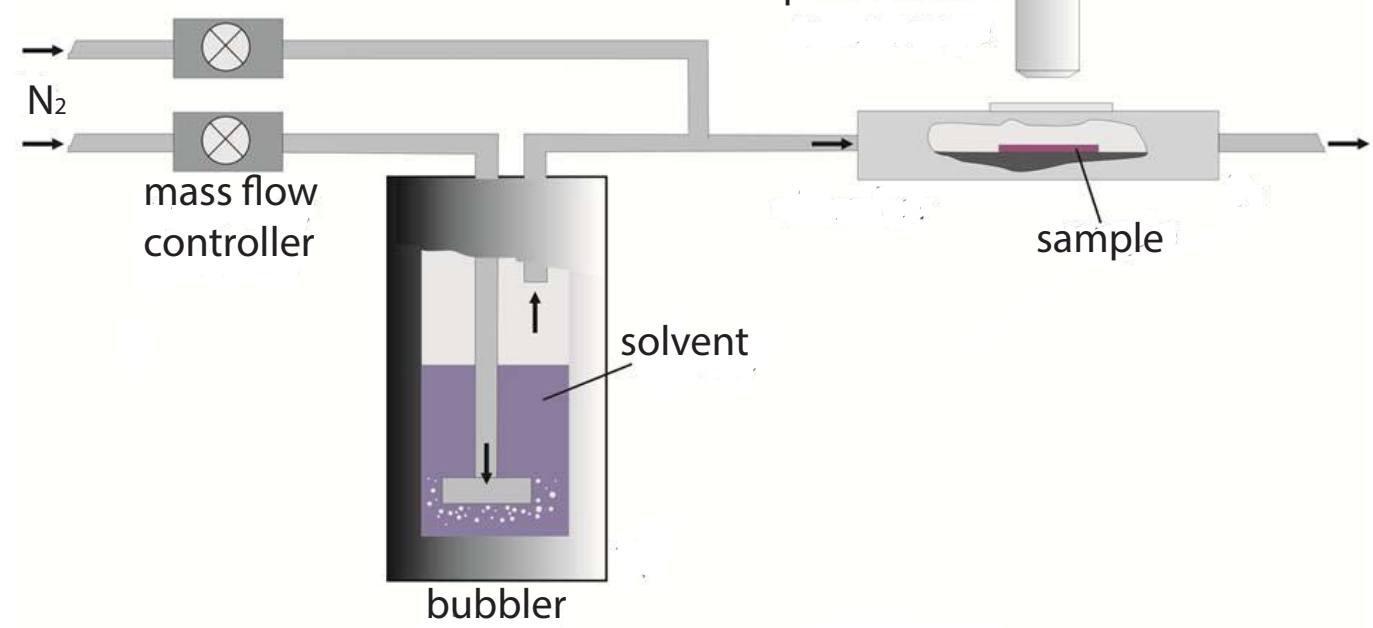

Figure 8.1: Schematic of the solvent vapour chamber setup. Courtesy of Sven Hüttner.

The vapour pressure was increased in $1 \%$ increments at intervals of 5 minutes to allow equilibration in the chamber. At $P_{\mathrm{v}} / P_{\text {sat }}=80 \%$ the water vapour condensed in the smaller gyroid producing a red shift in reflection and change in coloration (Figure 8.2), whereas the bigger gyroid were triggered at $P_{\mathrm{v}} / P_{\text {sat }}=$ 85\%. The corresponding reflection spectra are shown in Figure 8.3. 

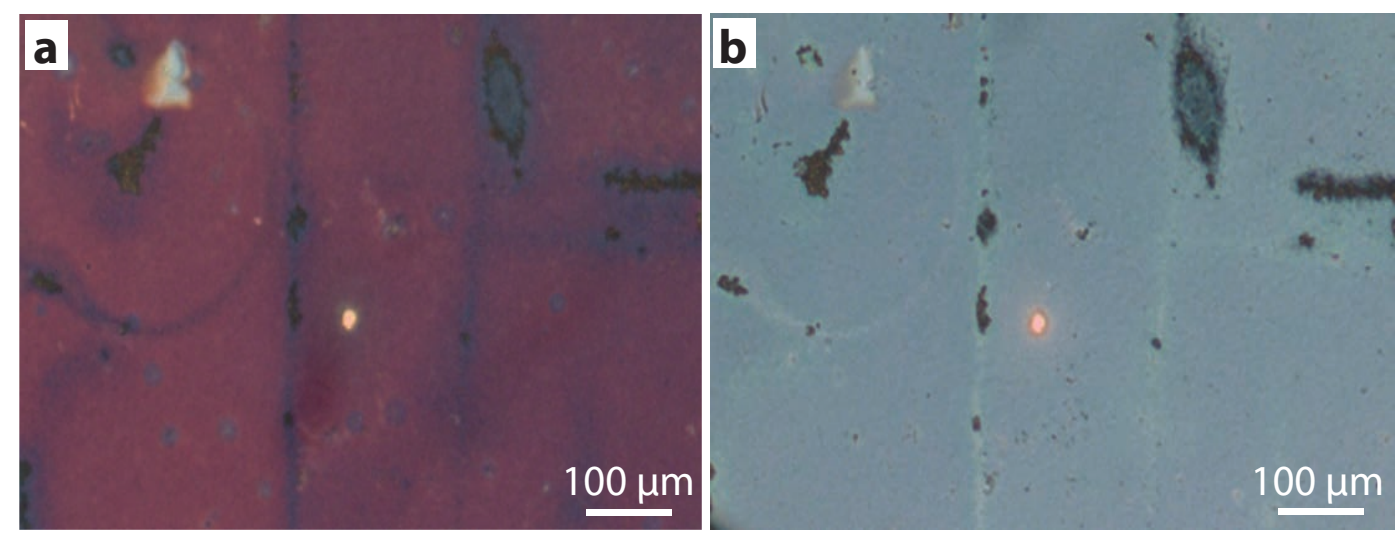

Figure 8.2: Optical microscope images of the $35 \mathrm{~nm}$ unit cell gyroid before (a) and after (b) water vapour condensation.

A small hysteresis was found when decreasing the vapour pressure with water desorption occurring at vapour pressure $2 \%$ lower than for adsorption. This deviation is characteristic for non-uniform pore geometries? where the adsorption takes place when the equilibrium pressure satisfies the larger pore section and the desorption is controlled by the smaller radius. It is reasonable to assume that the the variation of pore cross-section across the gyroid unit cell lies at the origin of the small experimental hysteresis.

The sensing response could not be directly measured because of the dimension of the chamber and the small flow rates. However, the desorption process was immediate (less than 1 second) upon the opening of the chamber, proving the very fast response of this sensing system.

Similar tests were performed with toluene vapour. In this case the smaller gyroid detected a vapour pressure of $P_{\mathrm{v}} / P_{\text {sat }}=60 \%$ and the bigger gyroid of $75 \%$ (Figure 8.4).

These results can be compared with the values from Eq. 8.1. The surface tensions of water and toluene were 72.8 and $28.4 \mathrm{mN} / \mathrm{m}$ ?, respectively. The mean pore curvature was approximated as one-half the difference of gyroid unit cell and strut thickness. As shown in Table 8.1 the capillary condensation was expected to occur at higher vapour pressures. This can be addressed by modifying the radius of curvature approximation. The gyroid pores were approximated as 


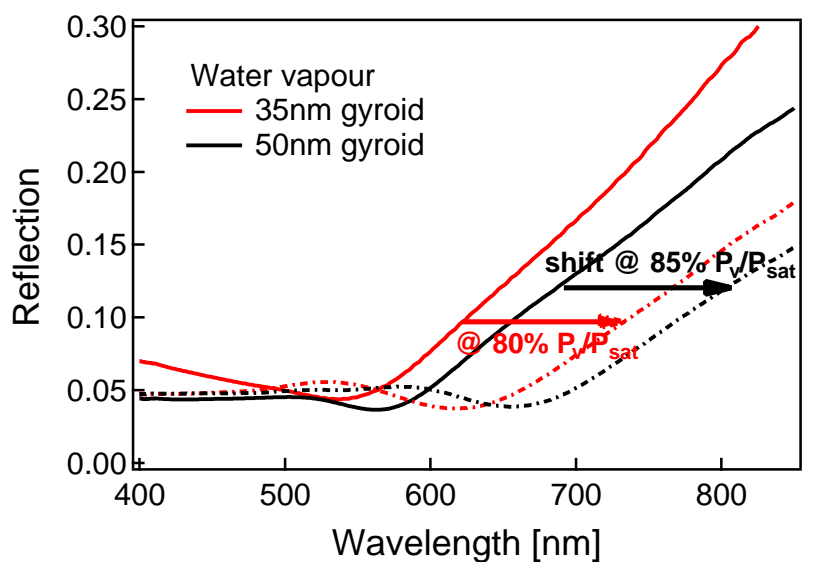

Figure 8.3: Reflection spectra of the gyroid metamaterial with increasing water vapour pressure. The capillary condensation and consequent reflection shift occur at different vapour pressures in the smaller and bigger gyroid.

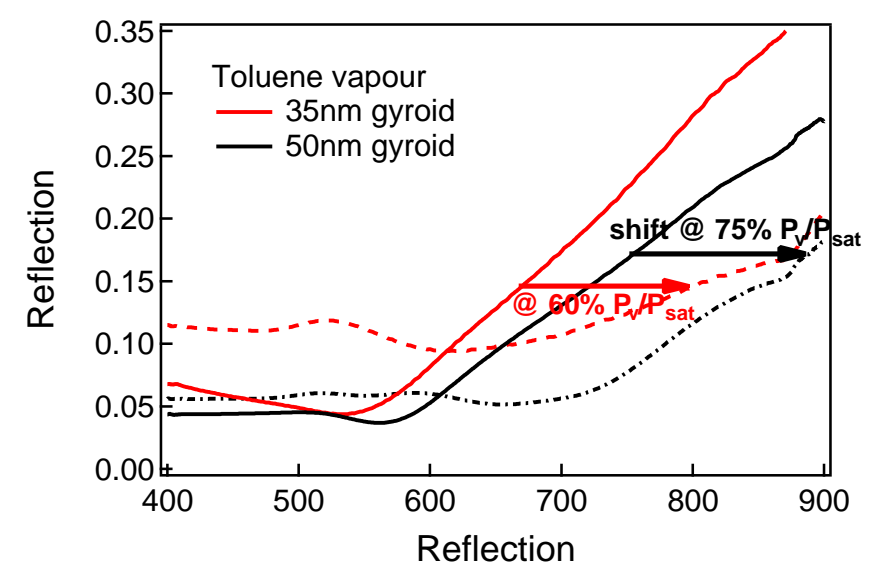

Figure 8.4: Reflection spectra of the gyroid metamaterial with increasing toluene vapour pressure. The capillary condensation and consequent reflection shift occur at different vapour pressures in the smaller and bigger gyroid.

cylinders, whereas Kelvin equation should be modified to take into account the gyroid pore geometry.

However, as found experimentally, lower surface tension decreases the vapour pressure of capillary condensation and increases its variation with pore size. In industrial applications a number of monitored vapours have low surface tensions, 
such as ammonia, benzene, formaldehyde, sulfur dioxide, supporting the appeal of this gyroid metamaterial sensor.

\begin{tabular}{llcc}
\hline \multirow{2}{*}{ Vapour } & Unit cell size & \multicolumn{2}{c}{$P_{\mathrm{v}} / P_{\text {sat }}$} \\
\cline { 3 - 4 } Water & $35 \mathrm{~nm}$ & $80 \%$ & $86 \%$ \\
& $50 \mathrm{~nm}$ & $85 \%$ & $91 \%$ \\
\multirow{2}{*}{ Toluene } & $35 \mathrm{~nm}$ & $60 \%$ & $71 \%$ \\
& $50 \mathrm{~nm}$ & $75 \%$ & $81 \%$ \\
\hline
\end{tabular}

Table 8.1: Comparison of experimental and predicted values of vapour pressures sensed by gyroids with different unit cell sizes.

\subsection{Conclusions}

The application of an optical metamaterial as vapour sensor was successfully proven. Environmental parameters strongly affect the optical response of the optical metamaterial and it was here exploited to monitor the concentration of water and toluene vapours. This sensor system may be applied to a number of other solvents, tuning the sensing response by the initial block copolymer molecular weight. 


\section{Appendix}

An incremental element of a beam is shown in Figure 5 for undeformed and deformed conditions. By definition the normal strain along $\Delta s$ is detemined as:

$$
\epsilon=\lim _{\Delta s \rightarrow 0} \frac{\Delta s^{\prime}-\Delta s}{\Delta s},
$$

The strain can be represented in terms of distance $y$ from the neutral axis and radius of curvature $\rho$. Since $\Delta \theta$ defines the angle between the cross sectional sides of the incremental elements, $\Delta s=\Delta x=\rho \Delta \theta$. The deformed length of $\Delta s$ becomes $\Delta s^{\prime}=(\rho-y) \Delta \theta$. Introducing these relations into Eq. 2 gives:

$$
\epsilon=\lim _{\Delta s \rightarrow 0} \frac{(\rho-y) \Delta \theta-\rho \Delta \theta}{\rho \Delta \theta}=\frac{-y}{\rho},
$$




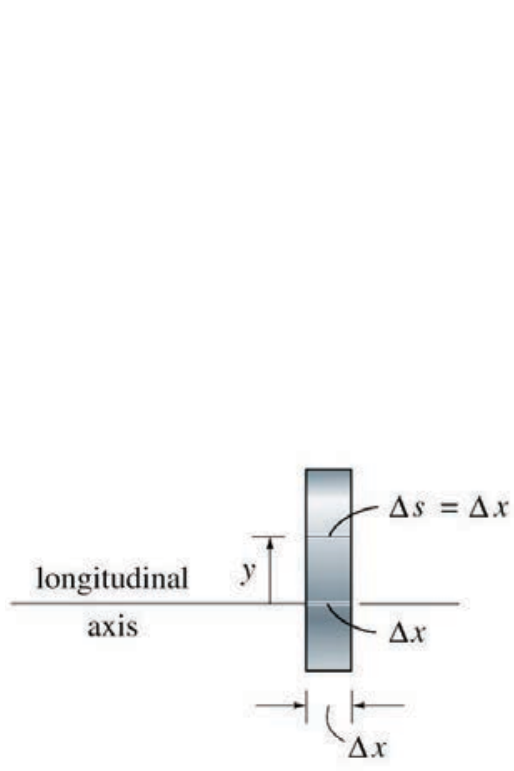

Undeformed element

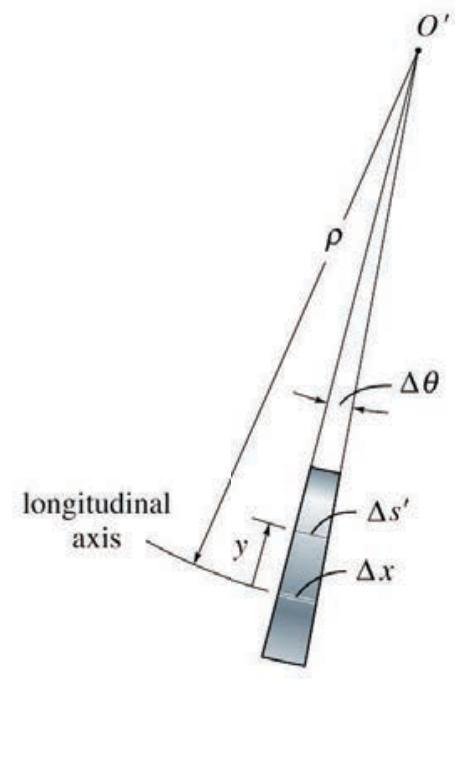

Deformed element

Figure 5: Undeformed and deformed elements in a beam. The longitudinal axis is set with respect to the neutral axis. 


\section{References}

P KRATOCHVfL. Glossary of basic terms in polymer science. 1996.

Ian W Hamley et al. The physics of block copolymers, volume 19. Oxford University Press New York, 1998.

Owen W Webster. Living polymerization methods. Science, 251(4996):887-893, 1991.

Paul J. Flory. Thermodynamics of High Polymer Solytions. Journal of Chemical Physics, 10:51-61, 1942.

Frank S Bates and Glenn H Fredrickson. Block Copolymers - Designer Soft Materials. Physics Today, (February):32-38, 1999.

Yiying Wu, Guosheng Cheng, Kirill Katsov, Scott W Sides, Jianfang Wang, Jing Tang, Glenn H Fredrickson, Martin Moskovits, and Galen D Stucky. Composite mesostructures by nano-confinement. Nature materials, 3(11):816-822, 2004.

Umang Nagpal, Francois A Detcheverry, Paul F Nealey, and Juan J de Pablo. Morphologies of linear triblock copolymers from monte carlo simulations. Macromolecules, 44(13):5490-5497, 2011.

TA Shefelbine, ME Vigild, MW Matsen, DA Hajduk, MA Hillmyer, EL Cussler, and FS Bates. Core-shell gyroid morphology in a poly (isoprene-block-styreneblock-dimethylsiloxane) triblock copolymer. Journal of the American Chemical Society, 121(37):8457-8465, 1999.

Wei Zheng and Zhen-Gang Wang. Morphology of abc triblock copolymers. Macromolecules, 28(21):7215-7223, 1995. 
Sang Soon Oh, Angela Demetriadou, Sebastian Wuestner, and Ortwin Hess. On the origin of Chirality in Nanoplasmonic Gyroid Metamaterials. Advanced Materials, page Doi: 10.1002/adma.201202788, October 2012.

Alan H. Schoen. infinite periodic minimal surfaces withouty self-intersections. Nasa technical note, D(5541), 1970.

Maik Rudolf Johann Scherer. Double-Gyroid-Structured Functional Materials: Synthesis and Applications. Springer Science \& Business, 2013.

Karsten Große-Brauckmann and Wohlgemuth Meinhard. The gyroid is embedded and has constant mean curvature companions. Calculus of Variations and Partial Differential Equations, 4(6):499-523, 1996.

William Longley and Thomas J McIntosh. A bicontinuous tetrahedral structure in a liquid-crystalline lipid. 1983.

K Michielsen and DG Stavenga. Gyroid cuticular structures in butterfly wing scales: biological photonic crystals. Journal of The Royal Society Interface, 5(18):85-94, 2008.

Vinodkumar Saranathan, Chinedum O Osuji, Simon G J Mochrie, Heeso Noh, Suresh Narayanan, Alec Sandy, Eric Dufresne, and Richard O. Prun. Structure, function, and self-assembly of single network gyroid (14132) photonic crystals in butterfly wing scales. pnas, 107(26):11676-11681, 2010.

Stephen Hyde, Z Blum, T Landh, S Lidin, BW Ninham, S Andersson, and K Larsson. The language of shape: the role of curvature in condensed matter: physics, chemistry and biology. Access Online via Elsevier, 1996.

Maik R J Scherer, Li Li, Pedro M S Cunha, Oren A Scherman, and Ullrich Steiner. Enhanced Electrochromism in Gyroid-Structured Vanadium Pentoxide. Advanced materials Deerfield Beach Fla, 24(9):1217-21, 2012.

Rachel a. Segalman. Patterning with block copolymer thin films. Materials Science and Engineering: R: Reports, 48(6):191-226, February 2005. 
Alexander C Edrington, Augustine M Urbas, Peter DeRege, Cinti X Chen, Timothy M Swager, Nikos Hadjichristidis, Maria Xenidou, Lewis J Fetters, John D Joannopoulos, Yoel Fink, et al. Polymer-based photonic crystals. Advanced Materials, 13(6):421-425, 2001.

Takeji Hashimoto, Kiyoharu Tsutsumi, and Yoshinori Funaki. Nanoprocessing based on bicontinuous microdomains of block copolymers: Nanochannels coated with metals. Langmuir, 13(26):6869-6872, 1997.

Cheolmin Park, Jongseung Yoon, and Edwin L. Thomas. Enabling nanotechnology with self assembled block copolymer patterns. Polymer, 44(22):6725-6760, October 2003.

Han-Yu Hsueh, Yen-Chun Huang, Rong-Ming Ho, Chih-Huang Lai, Taichi Makida, and Hirokazu Hasegawa. Nanoporous gyroid nickel from block copolymer templates via electroless plating. Advanced materials (Deerfield Beach, Fla. ), 23(27):3041-6, July 2011.

Di Wei, Maik RJ Scherer, Chris Bower, Piers Andrew, Tapani Ryhanen, and Ullrich Steiner. A nanostructured electrochromic supercapacitor. Nano letters, 12(4):1857-1862, 2012.

Edward JW Crossland, Marleen Kamperman, Mihaela Nedelcu, Caterina Ducati, Ulrich Wiesner, Detlef-M Smilgies, Gilman ES Toombes, Marc A Hillmyer, Sabine Ludwigs, Ullrich Steiner, et al. A bicontinuous double gyroid hybrid solar cell. Nano letters, 9(8):2807-2812, 2008.

Li Li, Lars Schulte, Lydia D Clausen, Kristian M Hansen, Gunnar E Jonsson, and Sokol Ndoni. Gyroid nanoporous membranes with tunable permeability. ACS nano, 5(10):7754-7766, 2011.

Victor G. Veselago. The Electrodynamics of Substances with Simultaneously Negative Values of epsilon and mu. Soviet Physics Uspekhi, 10(4):509-514, 1968.

Jb Pendry. Negative refraction makes a perfect lens. Physical review letters, 85(18):3966-9, October 2000. 
J B Pendry, D Schurig, and D R Smith. Controlling electromagnetic fields. Science (New York, N.Y.), 312(5781):1780-2, June 2006.

Ta-Jen Yen, WJ Padilla, N Fang, DC Vier, DR Smith, JB Pendry, DN Basov, and X Zhang. Terahertz magnetic response from artificial materials. Science, 303(5663):1494-1496, 2004.

J.B. Pendry, a.J. Holden, D.J. Robbins, and W.J. Stewart. Magnetism from conductors and enhanced nonlinear phenomena. IEEE Transactions on Microwave Theory and Techniques, 47(11):2075-2084, 1999.

Dr Smith, Wj Padilla, Dc Vier, Sc Nemat-Nasser, and S Schultz. Composite medium with simultaneously negative permeability and permittivity. Physical review letters, 84(18):4184-7, May 2000.

Wei Wu, Zhaoning Yu, Shih-Yuan Wang, R Stanley Williams, Yongmin Liu, Cheng Sun, Xiang Zhang, Evgenia Kim, Y Ron Shen, and Nicholas X Fang. Midinfrared metamaterials fabricated by nanoimprint lithography. Applied physics letters, 90(6):063107-063107, 2007.

J Zhou, Th Koschny, M Kafesaki, EN Economou, JB Pendry, and CM Soukoulis. Saturation of the magnetic response of split-ring resonators at optical frequencies. Physical review letters, 95(22):223902, 2005.

Shuang Zhang, Wenjun Fan, NC Panoiu, KJ Malloy, RM Osgood, and SR Brueck. Optical negative-index bulk metamaterials consisting of $2 \mathrm{~d}$ perforated metaldielectric stacks. Optics express, 14(15):6778-6787, 2006.

N Katsarakis, G Konstantinidis, A Kostopoulos, RS Penciu, TF Gundogdu, M Kafesaki, EN Economou, Th Koschny, and CM Soukoulis. Magnetic response of split-ring resonators in the far-infrared frequency regime. Optics Letters, 30(11):1348-1350, 2005.

Anan Fang, Thomas Koschny, and Costas M Soukoulis. Optical anisotropic metamaterials: Negative refraction and focusing. Physical Review B, 79(24):245127, 2009. 
Anthony J Hoffman, Leonid Alekseyev, Scott S Howard, Kale J Franz, Dan Wasserman, Viktor A Podolskiy, Evgenii E Narimanov, Deborah L Sivco, and Claire Gmachl. Negative refraction in semiconductor metamaterials. Nature materials, 6(12):946-950, 2007.

D Bruce Burckel, Joel R Wendt, Gregory A Ten Eyck, James C Ginn, A Robert Ellis, Igal Brener, and Michael B Sinclair. Micrometer-scale cubic unit cell 3d metamaterial layers. Advanced Materials, 22(44):5053-5057, 2010.

Stanley P Burgos, Rene de Waele, Albert Polman, and Harry A Atwater. A singlelayer wide-angle negative-index metamaterial at visible frequencies. Nature Materials, 9(5):407-412, 2010.

Durdu O Güney, Thomas Koschny, and Costas M Soukoulis. Intra-connected three-dimensionally isotropic bulk negative index photonic metamaterial. $O p$ tics express, 18(12):12348-53, June 2010.

Carsten Rockstuhl, Falk Lederer, Christoph Etrich, Thomas Pertsch, and Toralf Scharf. Design of an Artificial Three-Dimensional Composite Metamaterial with Magnetic Resonances in the Visible Range of the Electromagnetic Spectrum. Physical Review Letters, 99(017401):1-4, 2007.

Costas M. Soukoulis and Martin Wegener. Past achievements and future challenges in the development of three-dimensional photonic metamaterials. Nature Photonics, 5(SEPTEMBER), July 2011.

J B Pendry. A chiral route to negative refraction. Science (New York, N.Y.), 306(5700):1353-5, November 2004.

Rongkuo Zhao, Thomas Koschny, and Costas M Soukoulis. Chiral metamaterials: retrieval of the effective parameters with and without substrate. Optics Express, 18(14):14553-14567, 2010.

E Plum, J Zhou, J Dong, V A Fedotov, T Koschny, C M Soukoulis, and N I Zheludev. Metamaterial with negative index due to chirality. Physical Review B, 79(035407):1-6, 2009. 
Jiangfeng Zhou, Jianfeng Dong, Thomas Koschny, Maria Kafesaki, and Costas M Soukoulis. Negative refractive index due to chirality. arXiv preprint arXiv:0907.1121, 2009.

M Decker, M Ruther, C E Kriegler, J Zhou, C M Soukoulis, S Linden, and M Wegener. Strong optical activity from twisted-cross photonic metamaterials. Optics letters, 34(16):2501-3, August 2009.

Jiangfeng Zhou, Antoinette Taylor, John O'Hara, Roy Chowdhury, Rongkuo Zhao, and Costas M Soukoullis. Chiral thz metamaterial with tunable optical activity. Technical report, Los Alamos National Laboratory (LANL), 2010.

M Decker, R Zhao, CM Soukoulis, S Linden, and M Wegener. Twisted split-ringresonator photonic metamaterial with huge optical activity. Optics letters, 35(10):1593-1595, 2010.

Ahmadreza Hajiaboli, Mojtaba Kahrizi, and Vo-Van Truong. Optical behaviour of thick gold and silver films with periodic circular nanohole arrays. Journal of Physics D: Applied Physics, 45(48):485105, 2012.

William L Barnes, Alain Dereux, and Thomas W Ebbesen. Surface plasmon subwavelength optics. Nature, 424(6950):824-830, 2003.

Kahyun Hur, Yan Francescato, Vincenzo Giannini, Stefan a Maier, Richard G Hennig, and Ulrich Wiesner. Three-dimensionally isotropic negative refractive index materials from block copolymer self-assembled chiral gyroid networks. Angewandte Chemie (International ed. in English), 50(50):11985-9, December 2011.

Silvia Vignolini, Nataliya a Yufa, Pedro S Cunha, Stefan Guldin, Ilia Rushkin, Morgan Stefik, Kahyun Hur, Ulrich Wiesner, Jeremy J Baumberg, and Ullrich Steiner. A 3D optical metamaterial made by self-assembly. Advanced materials (Deerfield Beach, Fla.), 24(10):OP23-7, March 2012.

a. Knoll, a. Horvat, K. Lyakhova, G. Krausch, G. Sevink, a. Zvelindovsky, and R. Magerle. Phase Behavior in Thin Films of Cylinder-Forming Block Copolymers. Physical Review Letters, 89(3):035501, June 2002. 
Edward J. W. Crossland, Sabine Ludwigs, Marc a. Hillmyer, and Ullrich Steiner. Control of gyroid forming block copolymer templates: effects of an electric field and surface topography. Soft Matter, 6(3):670, 2010.

C.E. Wilkes. PVC Handbook. 2005.

Naoki Sakamoto and Takeji Hashimoto. Ordering Dynamics of a Symmetric Polystyrene-block-polyisoprene . 2 . Real-Space Analysis on the Formation of Lamellar Microdomain. Macromolecules, 3(98):3815-3823, 1998.

Krzysztof Matyjaszewski and Jianhui Xia. Atom Transfer Radical Polymerization. Chemical Reviews, 101(9):2921-2990, September 2001.

Nahrain E Kamber, Wonhee Jeong, Robert M Waymouth, Russell C Pratt, Bas G G Lohmeijer, and James L Hedrick. Organocatalytic ring-opening polymerization. Chemical reviews, 107(12):5813-40, December 2007.

Andrew S Zalusky, Roberto Olayo-Valles, Johanna H Wolf, and Marc a Hillmyer. Ordered nanoporous polymers from polystyrene-polylactide block copolymers. Journal of the American Chemical Society, 124(43):12761-73, October 2002.

Edward J W Crossland, Pedro Cunha, Sabine Ludwigs, Marc a Hillmyer, and Ullrich Steiner. In situ electrochemical monitoring of selective etching in ordered mesoporous block-copolymer templates. ACS applied materials $\&$ interfaces, 3(5):1375-9, May 2011.

D.R. Barbero, M.S.M. Saifullah, P. Hoffmann, H.J. Mathieu, D. Anderson, G.a.C. Jones, M.E. Welland, and U. Steiner. High-Resolution Nanoimprinting with a Robust and Reusable Polymer Mold. Advanced Functional Materials, 17(14):2419-2425, September.

David B Williams and C Barry Carter. The Transmission Electron Microscope. Springer, 1996.

K. Fuchs. The conductivity of thin metallic films according to the electron theory of metals. Mathematical Proceedings of the Cambridge Philosophical Society, 34:100-108, 11938. 
C Durkan and ME Welland. Size effects in the electrical resistivity of polycrystalline nanowires. Physical review B, 61(20):14215, 2000.

E Hi Sondheimer. The mean free path of electrons in metals. Advances in Physics, 1(1):1-42, 1952.

J B Pendry, a J Holden, D J Robbins, and W J Stewart. Low frequency plasmons in thin-wire structures. Journal of Physics: Condensed Matter, 10(22):47854809, June 1998.

Ardavan F Oskooi, David Roundy, Mihai Ibanescu, Peter Bermel, J D Joannopoulos, and Steven G Johnson. Meep: A flexible free-software package for electromagnetic simulations by the FDTD method. Computer Physics Communications, 181(3):687-702, 2010.

L Kuipers, MS Hoogeman, and JWM Frenken. Step dynamics on au (110) studied with a high-temperature, high-speed scanning tunneling microscope. Physical review letters, 71(21):3517, 1993.

Gert Ehrlich. Direct observations of the surface diffusion of atoms and clusters. Surface Science, 246(1):1-12, 1991.

Philippe Tassin, Thomas Koschny, and Costas M. Soukoulis. Effective material parameter retrieval for thin sheets: Theory and application to graphene, thin silver films, and single-layer metamaterials. Physica B: Condensed Matter, pages 1-4, January 2012.

Marion E Franke, Tobias J Koplin, and Ulrich Simon. Metal and metal oxide nanoparticles in chemiresistors: does the nanoscale matter? Small, 2(1):36-50, 2006.

Stefano Salvatore, Angela Demetriadou, Silvia Vignolini, Sang Soon Oh, Sebastian Wuestner, Nataliya a Yufa, Morgan Stefik, Ulrich Wiesner, Jeremy J Baumberg, Ortwin Hess, and Ullrich Steiner. Tunable 3D extended selfassembled gold metamaterials with enhanced light transmission. Advanced materials (Deerfield Beach, Fla.), 25(19):2713-6, May 2013. 
Angela Demetriadou, Sang Soon Oh, S. Wuestner, and Ortwin Hess. A trihelical model for gyroid metamaterials. New Journal of Physics, 14:DOI: 10.1088/1367-2630/14/8/083032, 2012.

Joseph Goldstein, Dale E Newbury, David C Joy, Charles E Lyman, Patrick Echlin, Eric Lifshin, Linda Sawyer, and Joseph R Michael. Scanning electron microscopy and X-ray microanalysis. Springer, 2003.

Juan Bautista González-Díaz, Antonio García-Martín, Gaspar Armelles, David Navas, Manuel Vázquez, Kornelius Nielsch, Ralf B Wehrspohn, and Ulrich Gösele. Enhanced magneto-optics and size effects in ferromagnetic nanowire arrays. Advanced Materials, 19(18):2643-2647, 2007.

Ventsislav K Valev, Alejandro V Silhanek, Werner Gillijns, Yogesh Jeyaram, Hanna Paddubrouskaya, Alexander Volodin, Claudiu G Biris, Nicolae C Panoiu, Ben De Clercq, Marcel Ameloot, et al. Plasmons reveal the direction of magnetization in nickel nanostructures. ACS nano, 5(1):91-96, 2010.

KB Crozier, A Sundaramurthy, GS Kino, and CF Quate. Optical antennas: Resonators for local field enhancement. Journal of Applied Physics, 94(7):46324642, 2003.

Carly S Levin, Cristina Hofmann, Tamer A Ali, Anna T Kelly, Emilia Morosan, Peter Nordlander, Kenton H Whitmire, and Naomi J Halas. Magnetic- plasmonic core- shell nanoparticles. Acs Nano, 3(6):1379-1388, 2009.

Vasily V Temnov, Gaspar Armelles, Ulrike Woggon, Dmitry Guzatov, Alfonso Cebollada, Antonio Garcia-Martin, Jose-Miguel Garcia-Martin, Tim Thomay, Alfred Leitenstorfer, and Rudolf Bratschitsch. Active magneto-plasmonics in hybrid metal-ferromagnet structures. Nature Photonics, 4(2):107-111, 2010.

Qi Zhang, Tian Jiang, and Yijun Feng. Slow-light propagation in a cylindrical dielectric waveguide with metamaterial cladding. Journal of Physics D: Applied Physics, 44(47):475103, 2011.

Piran R Kidambi, Caterina Ducati, Bruno Dlubak, Damian Gardiner, Robert S Weatherup, Marie-Blandine Martin, Pierre Seneor, Harry Coles, and Stephan 
Hofmann. The parameter space of graphene chemical vapor deposition on polycrystalline cu. The Journal of Physical Chemistry C, 116(42):22492-22501, 2012.

M Endo, C Kim, K Nishimura, T Fujino, and K Miyashita. Recent development of carbon materials for li ion batteries. Carbon, 38(2):183-197, 2000.

M Endo, YA Kim, T Hayashi, K Nishimura, T Matusita, K Miyashita, and MS Dresselhaus. Vapor-grown carbon fibers (vgcfs): basic properties and their battery applications. Carbon, 39(9):1287-1297, 2001.

Seungwoo Lee, Seongnam Kim, Teun-Teun Kim, Yushin Kim, Muhan Choi, Seung Hoon Lee, Ju-Young Kim, and Bumki Min. Reversibly stretchable and tunable terahertz metamaterials with wrinkled layouts. Advanced Materials, 24(26):3491-3497, 2012. 1

Andrea Di Falco, Martin Ploschner, and Thomas F Krauss. Flexible metamaterials at visible wavelengths. New Journal of Physics, 12(11):113006, 2010. 1

SMA Kazimi. Solid mechanics. Tata McGraw-Hill Education, 2001. 6

Mark G Allen. Diode laser absorption sensors for gas-dynamic and combustion flows. Measurement Science and Technology, 9(4):545, 1998. 9

P Laj, J Klausen, Merete Bilde, C Plaß-Duelmer, G Pappalardo, Cathy Clerbaux, U Baltensperger, J Hjorth, D Simpson, S Reimann, et al. Measuring atmospheric composition change. Atmospheric environment, 43(33):5351-5414, 2009. 9

David Smith and Patrik Španěl. The challenge of breath analysis for clinical diagnosis and therapeutic monitoring. Analyst, 132(5):390-396, 2007. 9

Laurier Lincoln Schramm. The language of colloid and interface science: A dictionary of terms. American Chemical Society, 1993. 10 
M Kruk, M Jaroniec, and A Sayari. Application of large pore mcm-41 molecular sieves to improve pore size analysis using nitrogen adsorption measurements. Langmuir, 13(23):6267-6273, 1997. 10

Minoru Miyahara, Hideki Kanda, Tomohisa Yoshioka, and Morio Okazaki. Modeling capillary condensation in cylindrical nanopores: a molecular dynamics study. Langmuir, 16(9):4293-4299, 2000. 10

Robert J Hunter, Lee R White, and Derek YC Chan. Foundations of colloid science, volume 1. Clarendon Press Oxford, 1987. 12

William M Haynes, David R Lide, and Thomas J Bruno. CRC Handbook of Chemistry and Physics 2012-2013. CRC press, 2012. 12 